\title{
FREE COMMUNICATION
}

\section{FC_01}

\author{
A randomised comparison of microwave endometrial ablation \\ with transcervical resection \\ of the endometrium; follow up at a minimum \\ of 10 years \\ A. Sambrook, K. Cooper \\ Aberdeen Royal Infirmary, Aberdeen, UK
}

Objective: To compare long term outcomes following microwave endometrial ablation $\left(\mathrm{MEA}^{\mathrm{TM}}\right)$ or transcervical resection of the endometrium (TCRE). Outcome measures include women's satisfaction with and acceptability of treatment, menstrual symptoms, changes in health related quality of life, and additional treatments received.

Materials and methods: Two hundred sixty-three women were followed-up using postal questionnaires at a minimum of 10 years following a randomised controlled trial comparing $\mathrm{MEA}^{\mathrm{TM}}$ and TCRE in a large UK teaching hospital. Analysis was performed as intention to treat for acceptability and satisfaction. Further surgery was obtained via a search of the hospital operative database.

Results: One hundred eighty-nine of the original 263 women returned questionnaires $(71 \%)$ after a minimum of 10 years post treatment. Women allocated to microwave ablation were significantly more likely to be totally or generally satisfied with treatment $(59.7 \%$ vs $51.5 \%$; $-037,0.20)$, to find it acceptable $(69.8 \%$ vs $62.5 \% ;-0.41,0.19)$, and would recommend it $(69.8 \%$ vs $61.0 \% ;-0.27,0.20)$. Six of the eight health related quality of life dimensions measured using Short Form 36 were improved following $\mathrm{MEA}^{\mathrm{TM}}$, five for TCRE. Days lost to work and disruptions to leisure and sex life are highly significantly reduced by both procedures from baseline. Bleeding and pain scores were highly significantly and similarly reduced following both MEA ${ }^{\mathrm{TM}}$ and TCRE, achieving amenorrhoea rates of $60.5 \%$ and $61.8 \%$ respectively. The hysterectomy rate after a minimum of 10 years was $17 \%$ in the MEA $^{\mathrm{TM}}$ and $28 \%$ in the TCRE arm.

Conclusions: Both techniques achieve significant and comparable improvements in menstrual symptoms, and health related quality of life. Whilst high rates of satisfaction and acceptability of treatment are achieved by TCRE, these are lower than levels following MEA ${ }^{\mathrm{TM}}$. This long term data when combined with the trials' operative findings and known costs of both procedures, now informs us that MEA ${ }^{\mathrm{TM}}$ is a more effective and efficient treatment for heavy menstrual loss than TCRE.

\section{FC_02}

Ultrasound guided high intensity focused ultrasounds (US-gFUS) therapy for uterine fibroids. Initial experience and safety issues in a western country A. Pessarrodona ${ }^{1}$, J. Muxart ${ }^{1}$, J. Isern ${ }^{1}$, S. Peng ${ }^{2}$, G. Redondo ${ }^{1}$,

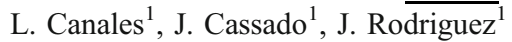

${ }^{1}$ Hospital Mutua de Terrassa, Terrassa, Spain, ${ }^{2}$ Clinical Center for Tumor Therapy,2nd affiliates Hospital of Chongqing Medical University, Chongqing, China

Introduction: HIFU is an emerging technology for thermal ablation of solid tumors, truly non-invasive, that is developing quickly. It has been used in Western countries to treat uterine fibroids guided with MRI since 2000, and its use was approved by the FDA in 2004, but in China and south-east Asia the use of HIFU for solid tumors began some years before, in 1997, guided by diagnostic US, with an overall of more than 6,000 patients treated from benign and malignant tumors. Since recent $\mathrm{CE}$ approval of these Chinese devices they are now also available for Western countries.

Objectives: We present the USgFUS technology for the treatment of uterine fibroids, report our study and treatment protocol, and safety issues of the procedure. Finally we give some preliminary data about imaging results and symptoms improvement.

Materials and methods: We included the first 20 patients treated with the HAIFU JC System from February 2008 until nowadays. All the patients had symptomatic fibroids, according to the UFSQOL questionnaire, but one. The mean maximum diameter was $7.1 \mathrm{~cm}$ and average volume $187 \mathrm{cc}$. Each patient was diagnosed by US and contrast enhanced MRI and all of them were closely followed-up, with a specially designed questionnaire regarding immediate postoperative pain, discomfort and recovery., about the possible discomfort in the first week after treatment, and 1 month after, this time together with a new UFS-QOL questionnaire, contrast-enhanced MRI and blood analysis.

Results: There were no clinically relevant complications during or immediately after treatment. There was one grade I skin burn, fully recovered 1 week after, one post-ablation febrile syndrome for 2 days and one spotty discharge for 3 days. All treatments were performed at high power with an average of $274 \mathrm{~W}$. The postoperative pain was $\mathrm{EVA}=0$ and all patients recovered to their normal life 24 to $48 \mathrm{~h}$ after the treatment. In the 1 month follow-up MRI $(n=12)$, the treated area covered more than $80 \%$ of the fibroid in most cases and some of the patients already show improvement in the UFS-QOL scores, no complications were found. 
Conclusions: We didn't find any clinically significant complications related to the procedure neither immediately nor in the 1 month follow-up. Patients recovered very soon after the procedure. Our imaging and symptomatic results are very promising but further studies and follow up need to be done.

\section{FC_03}

\section{Laparoscopic multiple myomectomy using intraoperative} ultrasound (IOUS)

D. Tutunaru, I. Luiza Condulescu, B. Alexandru, D. Florentina Lebit Elias Emergency Hospital, Bucharest, Romania

Aim: To investigate the efficacy of intraoperative ultrasound in detecting multiple uterine intramural leyomyomas.

Materials and methods: Prospective, randomized, group controlled study. Inclusion criteria: preoperative echographic demonstration of multiple uterine intramural leyomyomas. Exclusion criteria: cervical severe dysplasia, genital neoplasia. Intravaginal ultrasound was performed prior to operation using a VOLUSON 730 Expert BT-05 system with a 5-9 MHz RIC5-9 curved linear array volumetric real time $3 \mathrm{D}$ endovaginal probe. We used a laparoscopic ultrasound system "Sharplan u Sight 9010", which is a real time 2-D mechanical sector scanning diagnostic ultrasound system. The probe used was a 10-mm laparoscopic probe of $8 \mathrm{MHz}$. Laparoscopic system used was provided by Karl Storz. A total of 46 patients were included in this study. Patients were randomly divided in a study group of 25 and a control group of 21 subjects, after have signed IC. Laparoscopy was performed by one and the same surgeon, who didn't know the location and number of the fibroids. In the study group intraoperative scanning of the uterine surface was done in order to locate the fibroids and myomectomy was performed afterwards. In control group myomectomy was performed after using palpation and visualisation of external aspect of the uterine surface as guidance landmarks. We controlled postoperatively the existence of fibroids after 1 week and 6 months respectively.

Results: Of 46 patients enrolled in study, all matched inclusion criteria. In the study group there was no fibroid in any of the postoperative visits. In the control group we discovered nine cases of restant fibroids, ranging from 11 to $27 \mathrm{~mm}$ in the V1 (1 week) and 11 nodules ranging from $15 \mathrm{~mm}$ to $34 \mathrm{~mm}$ in V2 (6 months) Conclusions: Intraoperative ultrasound in cases of multiple intramural leyomyomas is a extremely helpful tool to use in order to have total removal of multiple fibroids, far better than simple visual inspection and palpation of the uterus.

\section{FC_04}

The impact of alternative treatment for abnormal uterine bleeding on hysterectomy rates in a tertiary referral centre H. van Dongen, A.van de Merwe, C. de Kroon, F.W. Jansen Leiden University Medical Center, Leiden, Netherlands

Introduction: The purpose of this study was to assess the influence of alternatives to hysterectomy for abnormal uterine bleeding (AUB) on hysterectomy rates.
Materials and methods: We performed a retrospective cohort study in a university hospital. The cohort consisted of all premenopausal patients attending our outpatient university clinic with abnormal uterine bleeding between January 1st 1995 and December 31st 2004. Their medical records were reviewed. Patients were identified based on (specific) diagnostic and therapy codes used in the registry system of the hospital. The total number of placements of levonorgestrel-IUD (LNG-IUD), hysteroscopic surgery and hysterectomies performed per year was estimated. Additionally, the course of treatment of each patient was assessed. Results: A total number of 795 surgical procedures were performed, whilst 269 LNG-IUDs were placed. The percentage of patients receiving surgery increased significantly $(p<0.001)$, from $27.5 \%$ in 1995 to $51.5 \%$ in 2004 . The endometrial ablation rate decreased significantly over time $(p<0.001)$, whereas hysteroscopic polyp or myoma removal and insertion of LNG-IUD both increased $(p<0.001)$. The proportion of hysterectomies for AUB increased significantly $(p<0.001)$ from $12.1 \%$ to $28.8 \%$.

Conclusion: Despite the introduction of alternative therapies, the hysterectomy rate in the management of AUB did not decrease in our clinic.

\section{FC_05}

Prospective randomised comparison of total laparoscopic hysterectomy (TLH) with laparoscopic assisted vaginal hysterectomy (LAVH)

I. Georgiev

Department of Gynecology and Obstetrics, Friedrich Schiller University Jena, Jena, Germany

Aim: To compare the both internationally established surgical procedures LAVH and TLH

Materials and methods: In the present prospective-randomised investigation the course of the illness of 100 women after hysterectomy was analysed. 50 patients underwent a LAVH, 50 were operated with the TLH-method. They were compared concerning the indications, the anamnesis data, the operation times and the uterine weights, the intra- and post-surgical complication rate, the consumption of analgesics, the decrease of haemoglobin and the stationary stay duration.

Results: The bleeding disturbances as well as the big and symptomatic uterus myomatosus were the most frequent indications for the hysterectomy. During the operation all 100 patients did not develop bladder-, bowel-, ureter- or vascularlesions. The rate of the post surgical complications was $8 \%$ for each group. The both set up operation technologies LAVH and TLH were equally safe for the patients. The operation time with the TLH was shorter than with the LAVH. The blood loss with the TLH was lower than with the LAVH. The consumption of analgesics after TLH was lower than after LAVH.

Conclusion: On account of the raised endoscopic-surgical effort a learning curve is a necessary requirement. In medical centres with modern surgical adjustment the both laparoscopic technologies replaced the abdominal hysterectomy almost completely. Basically the TLH is superior to the LAVH for patients with narrow vaginal relations and big uterus in every respect. 


\section{FC_06}

Prevalence of unexpected intra-uterine pathology diagnosed at office hysteroscopy in patients indicated for IVF J. Kasius $^{1}$, A. Timmermans ${ }^{1}$, J. van Disseldorp ${ }^{1}$, E. van Santbrink ${ }^{3}$, P. Devroey ${ }^{2}$, B.C. Fauser ${ }^{1}$, F. Broekmans ${ }^{1}$, H. Fatemi ${ }^{2}$

${ }^{I}$ University Medical Center, Department for Reproductive Medicine, Utrecht, Netherlands, ${ }^{2}$ Vrije Universiteit Brussels, Department for Reproductive medicine, Brussels, Belgium ${ }^{3}$ Erasmus MC, Department of Obstetrics and Gynecology, Rotterdam, Netherlands

Introduction: Implantation failure after IVF may be due to endometrial function, embryo quality or both. The prevalence of minor intracavitary pathology in cases with an apparent normal transvaginal sonography (TVS) observed at hysteroscopy has been recorded to be $25-40 \%$. Treatment of such pathology prior to initiating IVF/ICSI has been advocated without high-quality evidence. Objective: To evaluate the feasibility of office hysteroscopy and to evaluate the prevalence of minor intracavitary pathology at hysteroscopy in an asymptomatic population of IVF patients in whom TVS detected no pathology.

Materials and methods: This multicentered study was performed in a university hospital setting with office hysteroscopy facilities. Patients with a regular menstrual cycle, no prior hysteroscopy, presence of both ovaries and indicated for a first IVF/ICSI treatment were included. Patients underwent TVS in the early follicular phase of a cycle. In case of a normal TVS these patients were scheduled for hysteroscopy in the early-mid follicular phase of the cycle, 1 to 3 months before starting IVF/ICSI treatment. Vaginoscopic hysteroscopy was performed in an ambulatory office setting. The presence of predefined intracavitary pathology (polyps, myoma, synechia, septum, inflammation) was recorded and described in a standard manner.

Results: A total of 543 procedures were carried out. Failure to enter the cavity due to pain occurred in six cases $(1.1 \%)$. Investigation of the cavity could be completed in 537 cases. Endometrial polyps were identified in 31 cases (5.8\%), submucosal myomas in three $(0.6 \%)$. Cases with septum $(13,2.4 \%)$ and synechia $(8,1.5 \%)$ were also diagnosed. Histology confirmed endometritis in 17 cases $(3.2 \%)$. The overall prevalence of intracavitary pathology in this IVF/ICSI population was $12.6 \%$.

Conclusions: The observed prevalence of minor intracavitary pathology in an asymptomatic population of first IVF/ICSI patients is clearly lower than previously reported. Although hysteroscopy is very well feasible, this low prevalence of unexpected abnormalities does not justify the routine implementation of hysteroscopy in a population of IVF/ICSI patients.

\section{FC_07}

Cost-minimization analysis comparing outpatient see-and-treat hysteroscopy service with traditional hysteroscopy service models in the National Health Service England E. Saridogan ${ }^{1}$, D. Tilden ${ }^{2}$, N. Davis ${ }^{3}$, D. Sykes ${ }^{4}$, D. Subramanian ${ }^{3}$ ${ }^{I}$ University College London Hospital, London, UK, ${ }^{2}$ THEMA Consulting Pty Ltd, Sydney, NSW, Australia, ${ }^{3}$ Ethicon, Livingston, Scotland, UK, ${ }^{4}$ PRMA Consulting, Fleet, Hampshire, UK
Objective: To evaluate the economic impact of three different hysteroscopy service models.

Materials and methods: A decision-analytic model was constructed from the UK National Health Service (NHS) perspective, to compare the costs of; (1) an outpatient 'see-and-treat' hysteroscopy ("outpatient see-and-treat service"), (2) an outpatient diagnostic hysteroscopy followed by referral to operative hysteroscopy under general anaesthesia (GA) in theatre ("outpatient and referral service") and, (3) a traditional 'see-and-treat' hysteroscopy under GA ("GA see-and-treat service"). Probabilities of successful outpatient diagnostic hysteroscopy, and of successful treatment at the same visit in the "outpatient see-andtreat service" arm were based on the prospective analysis of 1,109 women who attended an outpatient see-and-treat hysteroscopy service. Hysteroscopy was performed between July 2001 and June 2007 at a tertiary hospital (University College London Hospital) using Gynecare Versascope* Hysteroscopy System/ Gynecare Versapoint* Bipolar Electrosurgery System. Expert opinion was used to extrapolate the probabilities to the other two hysteroscopy service models. Costs were estimated using the 2006-2007 NHS Reference costs. As repeat interventions would be carried out until successful removal of the pathology occurs in all three hysteroscopy service models, effectiveness was assumed to be the same. Therefore, a cost-minimization analysis was performed.

Results: Results from the cohort study indicate that $96.2 \%(1,067$ of 1,109) of the subjects in the "outpatient see-and-treat service" treatment arm completed a successful diagnostic hysteroscopy examination. Of these, $29.7 \%$ (317 of 1,067) had see-and-treat procedures, and the most common indications were polyps or fibroids. Total costs were lowest with "outpatient see-and-treat service" ( $£ 409$ per patient). Costs of the "outpatient and referral service" and "GA see-and-treat service" were $£ 420$ and $£ 711$ per patient respectively. The lower cost of the "outpatient see-and-treat service" was observed across a number of patient subgroups (age, menopause status, indication) and when subjected to sensitivity analyses.

Conclusion: Of the three hysteroscopy service models evaluated, outpatient see-and-treat hysteroscopy was associated with the least treatment costs. Therefore, this service model may reduce the total cost of care in women referred for hysteroscopy.

\section{FC_08}

Proximal tubal occlusion of hydrosalpinges with the Essure ${ }^{\circledR}$ device before in vitro fertilisation in an outpatient setting. A first or a second choice?

A. Ubeda, M. Mascaro, O. Salas

${ }^{1}$ Institut Universitari Dexeus, Barcelona, Spain, ${ }^{2}$ Hospital Son Dureta, Palma de Mallorca, Spain, ${ }^{3}$ Institut Universitari Dexeus, Barcelona, Spain

Introduction: World-wide references have demonstrated that both uni and bilateral hydrosalpinges may decrease success rates of in vitro fertilisation (IVF) procedures through several mechanisms. Any kind of salpingectomy has turned out to recover global IVF pregnancy rates. On the other hand, uneventful pregnancies and 
at-term deliveries have yet been published after Essure ${ }^{\circledR}$ placement. Nevertheless, Essure ${ }^{\circledR}$ blockage of hydrosalpinges seems to be left to women at-risk for surgery.

Design: Retrospective descriptive study in two Spanish university hospitals.

Materials and methods: Four women were referred for Essure ${ }^{\circledR}$ bilateral, proximal tubal occlusion either after ultrasound or hystersalpingography diagnosis of hydrosalpinx before IVF. As a rather new application of Essure ${ }^{\circledR}$ procedure, patients were also explained the possibility of laparoscopic surgical salpingectomy or tubal occlusion. They all signed a written consent for Essure ${ }^{\circledR}$ method. Potential and unavoidable complications such as infection, perforation, need of further surgery or lack of complete tubal blockage were explained. Patients were orally pretreated with one single dose of azitromyzin $1 \mathrm{~g}$ and metronidazol $500 \mathrm{mg}$ every $12 \mathrm{~h}$ for 5 days. As in all Essure ${ }^{\circledR}$ placements, they also were counselled to take one dose of ibuprofen $600 \mathrm{mg}$. Procedures were carried out in the office, through vaginoscopy and no complications were registered.

Results: Success rate was $100 \%$. Clinical follow-up did not reveal any sign of infection (pelvic pain or fever). Follow-up after 1 and 2 weeks showed no increase of hydrosalpinx/ges ultrasonographic size. At present, women are waiting for an IVF cycle after the third month of the microinserts placement, which was checked through pelvic X-ray and ultrasound.

Discussion: Essure ${ }^{\circledR}$ procedure seems to be an easier alternative to surgical salpingectomy or tubal occlusion in case of hydrosalpinges before IVF, without the risks of anaesthesia or operative complications. Furthermore, successful pregnancy outcomes time after the Essure ${ }^{\circledR}$ insertion have been referred. As a whole, justified expectancy is to be raised around this recent and promising application of the Essure ${ }^{\circledR}$ procedure.

\section{FC_09}

The feasibility of outpatient hysteroscopic sterilisation using the Essure ${ }^{\mathrm{TM}}{ }^{\circledR}$ system: an evaluation of patient and technical factors predictive of procedural success

M. Pathak, A. Coomaraswamy, J. Gupta, J. Clark

Birmingham Women's Hospital, Birmingham, UK

Design: Prospective cohort study

Materials and methods: Demographic and procedural data was collected prospectively from 251 consecutive women undergoing outpatient hysteroscopic sterilisation procedure without sedation or general anaesthesia. Multivariable logistic regression was used to identify demographic (BMI $>30$; Parity; previous Caesarean section) and operative technical factors (need for cervical dilatation; uterine size $>8$ weeks; uterine axis; vaginoscopic approach; surgeon) independently predictive of a successful bilateral placement of the Essure ${ }^{\circledR T M}$ microinsert.

Main outcome measures: Technical feasibility, operative complications and tubal occlusion at 3 months as confirmed by hysterosalpinography. Patient and operative factors predictive for procedural success (operator, BMI, parity, uterine size, axis, menstrual phase, presence of intrauterine pathology, cervical stenosis and use of vaginoscopic approach).
Results: Successful tubal placement of Essure ${ }^{\circledR T M}$ micro inserts was achieved in 234 of 251 (93\%, 95\% CI 89-96\%) of women. There were no major procedure-related complications, but transient vaso-vagal reactions occurred in 14 of 251 patients $(6 \%$, $95 \%$ CI 3-9\%). None of the demographic or operative technical factors identified were independently predictive for success of the outpatient sterilisation procedure on multivariable modelling

Conclusion: Outpatient hysteroscopic sterilisation using the Essure $^{\circledR T M}$ system without sedation or general anaesthesia is a successful and safe procedure. The successful bilateral placement of the Essure ${ }^{\circledR T M}$ does not appear to be influenced by patient, anatomic or operative factors. This contrast to an earlier published study by our group where secretory phase of the menstrual cycle and an enlarged uterus were associated with failed device placement. This contrasting finding may reflect improved surgical expertise with increased experience.

\section{FC_10}

The histopathologic significance of intrauterine lesions in asymptomatic postmenopausal patients

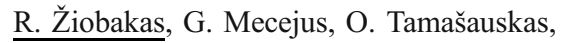

N. Bartaševiciute, E. Jakiunas, G. Drasutiene

Department of Gynecology, Clinic of Ob/Gyn of Vilnius University, Vilnius city University Hospital, Vilnius,

Lithuania

Objective: The objective of the study was to determine the pathologic significance of asymptomatic intrauterine lesions in bleeding-free postmenopausal women.

Materials and methods: A retrospective descriptive study of 249 postmenopausal women subjected to hysteroscopy with an ultrasound indication of asymptomatic uterine lesions during 2004-2007 was carried out. Data collected included age, parity, menopausal status, BMI, use of HRT, tamoxifen treatment in cases of breast cancer, other medical conditions associated with endometrial cancer (obesity, hypertension, diabetes) and the findings of ultrasound examination and hysteroscopy.

Results: Mean age of patients was 59 years (range 47-80) and mean postmenopausal age was 10 years (range 1-28). Mean BMI of patients was $29 \mathrm{~kg} / \mathrm{m}^{2}$ and $34 \%$ of all patients were obese $\left(\mathrm{BMI} \geq 30 \mathrm{~kg} / \mathrm{m}^{2}\right) ; 58 \%$ of patients had hypertension. $10 \%$ of patients were using tamoxifen as adjuvant treatment of breast cancer. Of the 249 patients with suspected intrauterine lesions, 215 focal lesions (polyps and myomas) were confirmed by hysteroscopy. The prevalence of malignancy or atypical hyperplasia was $2.8 \%$ : in three patients $(1.2 \%)$ the histologic diagnosis was endometrial adenocarcinoma located within the polyp, and in four patients $(1.6 \%)$ the diagnosis was atypical hyperplastic endometrial polyp. Complications associated to operative hysteroscopy were recorded: $2.4 \%$ of patients experienced a uterine perforation. Conclusion: The risk of endometrial carcinoma in bleedingfree postmenopausal women with asymptomatic intrauterine lesions is low. Nevertheless, because malignancy occurs, although rarely, and because the operative risk is low, hysteroscopy and polyp resection can be recommended in patients with low operative risk. 


\section{FC_11}

Narrow-band imaging and endometrial lesions

D. Surico, A. Vigone, R. Amadori, L. Leo

Maggiore della Carità Hospital, Novara, Italy

Introduction: Narrow-band imaging (NBI) is a novel endoscopic technique able to enhance the accuracy of diagnosis by using narrow-bandwidth filters in a red-green-blue sequential illumination system. This results in different images at distinct levels of the mucosa and increases the contrast between the epithelial surface and the subjacent vascular pattern. The aim of our study is to compare, referring as gold standard to histologic result, conventional hysteroscopy and hysteroscopy by using NBI in the detection of endometrial lesions.

Materials and methods: We assessed 100 postmenopausal patients affected by abnormal uterine bleeding and with endometrial thickness more than $4 \mathrm{~mm}$ at transvaginal ultrasound. In all patients, we performed a conventional hysteroscopy and applied NBI. Each hysteroscopy was first performed by a junior surgeon and then by an experienced endoscopist. Biopsies of suspected areas were send to the pathologist.

Results: In the detection of endometrial cancer, hysteroscopy by using NBI demonstrated a sensibility of $100 \%$ and conventional hysteroscopy a sensibility of $98 \%$. In endometrial hyperplasia, conventional hysteroscopy showed a sensibility of $56 \%$ while NBI had a sensibility of $75 \%$. Both in endometrial cancer and hyperplasia, NBI improves identification by junior surgeons (from $41.6 \%$ to $75 \%$ in hyperplasia and from $70 \%$ to $90 \%$ in cancer)

Discussion: In our study we experienced that NBI can be a useful additional methodology for early detection of endometrial lesions providing an increase of accuracy in visual identification of both endometrial cancer and hyperplasia by experienced endoscopists as well as by novices.

\section{FC_12}

Nurse hysteroscopists: a multi-centered audit evaluating current practice in the UK

G. Smith ${ }^{1}$, G. Steele ${ }^{l}$, S. Jones ${ }^{1}$, J. Pansini-murrell ${ }^{2}$

${ }^{I}$ B.S.G.E., London, UK, ${ }^{2}$ University of Bradford, Bradford, UK

Introduction: In the UK since 2001, nurses have undertaken academic and clinical training in diagnostic hysteroscopy. There are currently 36 qualified practicing nurse hysteroscopists with a further 24 in training. The B.S.G.E. accredits the training and nurses are required to register as members of the society and submit audit data for re-accreditation after 3 years. To date there are no nationally approved standards for what constitutes a successful diagnostic hysteroscopy and there is variation in failure rates reported in medical literature.

Materials and methods: This prospective audit will be undertaken for a minimum of 6 months commencing February 1st 2008. Data will be collected on the collection sheet following each procedure and sent to a central location for analysis. Each nurse will have been issued with an identification code and all cases will be de-identified and replaced by a number to ensure anonymity.
Results: The results will give an indication of hysteroscopy location, anaesthetics, instrumentation and distension media used, performance, failure rates, biopsy practices, age of clients and presenting complaints

Discussion: The results of this audit will not only allow this group of healthcare professionals to evaluate their practice and compare results with those reported in the medical literature, but will provide information relating to failure rates in postmenopausal as compared to pre-menopausal women. It has the potential to highlight areas of poor practice and the B.S.G.E. have mechanisms in place to manage such findings. It will provide data to establish a bench mark for future practice and audit and may provide guidelines for setting a gold standard in hysteroscopy performed by nurse hysteroscopists.

\section{FC_13}

How much do ultrasound scan findings correlate to hysteroscopic findings?

S. S M Wong $^{1}$, S.E. Jones ${ }^{2}$, S.Elton ${ }^{2}$

${ }^{1}$ Calderdale Royal Hospital, Halifax, UK, ${ }^{2}$ Bradford Royal Infirmary, Bradford, UK

Introduction: In women presenting with postmenopausal bleeding, it is of common practice to use ultrasound scan measurement of endometrial thickness as one of the criteria to assess whether diagnostic hysteroscopy is necessary. It is also not uncommon to offer ultrasound scan assessment as part of the one stop outpatient hysteroscopy clinic in hospitals in the UK. This audit aims to assess if there is any correlation between findings from ultrasound scan and hysteroscopy.

Materials and methods: Information on women who have diagnostic hysteroscopy at a one stop outpatient hysteroscopy clinic with a recent or "on the spot" pelvic ultrasound scan was collected retrospectively between 2003 and 2007. The data were stored and analysed using the SPSS software.

Results: Within 4.5 years, 2,928 women had a recent or "on the spot" pelvic scan and a diagnostic hysteroscopy performed at the clinic. Among 774 cases where fibroid was seen on scan, only 146 cases were confirmed by hysteroscopy, but 72 out of 2,244 cases with no fibroid seen on scan were found to have fibroid at hysteroscopy (negative predictive value $=97 \%$ ). Out of 280 cases where scan has shown intrauterine polyp, only 138 cases were confirmed by hysteroscopy. Among 2,738 cases where no intrauterine polyp were seen on scan, 550 cases were found to have polyp at hysteroscopy (negative predictive value=80\%). 77 pelvic scans were reported to have suspicious findings but only 18 cases of endometrial hyperplasia, cystic change and malignant looking endometrium were reported at hysteroscopy. However, 207 cases of endometrial hyperplasia, cystic changes and malignant looking endometrium were reported at hysteroscopy out of the 2,683 cases where their scan were not known to be suspicious (negative predictive value $=92 \%$ ). 530 out of 1,078 cases $(49 \%)$ with endometrial thickness of $5 \mathrm{~mm}$ or less were found to have genuine atrophic endometrium at hysteroscopy.

Conclusion: Ultrasound scan findings correlate very poorly with hysteroscopic findings, except in the absence of pathology. In 
women with normal clinical pelvic examination excluding cases of postmenopausal bleeding, it is debatable whether an ultrasound scan is necessary prior to hysteroscopy and its doubtful value on the patient's management.

\section{FC_14}

Diagnostic and therapeutic outpatient hysteroscopy M. Pathak ${ }^{1}$, C. Gulemser ${ }^{2}$, A. Shahid ${ }^{2}$, E. Saridogan ${ }^{2}$

${ }^{I}$ Birmingham Women's Hospital, Birmingham, UK, ${ }^{2}$ University College of London, London, UK

Introduction: Hysteroscopy is the gold standard method for investigation of intra-uterine abnormalities. With the advent of high resolution ultrasound examination the need for endoscopic assessment of uterine cavity is less frequently required. However, development of hysteroscopic techniques has enabled clinicians to perform these procedures in outpatient setting as well as utilising the operating narrow guage hysteroscope for therapeutic procedures. The purpose of this audit was to assess the feasibility of diagnostic and therapeutic OPD hysteroscopy.

Materials and methods: Data was collected prospectively from all the patients referred for outpatient hysteroscopy for various clinical reasons. Outpatient hysteroscopy was performed using $3.5 \mathrm{~mm}$ hysteroscope (Gynecare) using normal saline for distension. Informed consent was obtained before proceeding. When polyps were detected patients were given the option of polyp removal in the outpatient setting. Bipolar electrode was used for outpatient polyp removal and specimen was retrieved with a hysteroscopic grasping forceps. An endometrial biopsy was obtained from patients with abnormal uterine bleeding.

Results: Between July 2001 and June 2007 1,109 outpatient hysteroscopies were performed. The most common indications for referral for an outpatient hysteroscopy were menorrhagia $(29.8 \%)$, postmenopausal bleeding (25.1\%) and irregular periods $(14.5 \%)$ Hysteroscopic examination was successfully completed in $96.1 \%$ of the patients. The two most common pathologies found were intrauterine polyps (38.1\%) and submucosal fibroids (14.5\%). Histological examination showed 12 patients to have endometrial carcinoma; endometrial hyperplasia was detected in eight patients. Out of 422 patients who were found to have intrauterine polyps, $274(64.9 \%)$ had successful polyp removal in the outpatient setting using a bipolar electrode system.

Conclusion: Hysteroscopic examination is possible in majority of women. See-and-treat outpatient hysteroscopy appears to be a feasible and highly successful alternative to hysteroscopic procedures under general anaesthesia and should be offered as the first line option to patients who need a hysteroscopic examination of the uterine cavity.

\section{FC_15}

\author{
The effect of transobturator tape for treatment \\ on female sexual functioning \\ M. Jong, de, P. Dijkhuizen, K. Aalders, M. Balken van \\ Rijnstate Hospital, Arnhem, Netherlands
}

Introduction: Transobturator tape (TOT) may improve sexual function by decreasing incontinence, however it may worsen by the procedure itself. The aim of this study is to describe the effect of TOT on sexual functioning in women.

Materials and methods: A prospective observational study was performed. All patients underwent a tension-free TOT outside-in procedure (Uretex $\mathrm{TO}^{\circledR}$ ). They were invited to answer the Incontinence Quality of Life Questionnaire (I-QoL), the Urinary Distress Inventory (UDI) and the nine questions on Sexual Functioning (NSF-9), prior to surgery, 6 weeks and 12 months postoperatively.

Results: A total of 54 women, aged 37 to 78 , were included. Twenty-three women (43\%) were postmenopausal. Both the UDIscore and the I-QoL-score significantly improved after surgery. Forty women (78\%) were sexually active in the month prior to surgery. Forty percent of these women reported being sexual active once a week or more. Urinary loss during sexual activities was identified in 22 women (55\%). One fourth of the women had experienced lubrication problems, while $40 \%$ of the women never or just occasionally achieved an orgasm. Pain during and after sexual activity was mentioned by $55 \%$, of those women $12.5 \%$ experienced this pain frequently. $38 \%$ of the women had a satisfactory sexual life. No significant differences were reported on the frequency of sexual activity and problems with lubrication and orgasm 6 weeks postoperatively and 12 months after surgery. Urinary leakage during sexual activity was significantly improved after the TOT procedure. Six week postoperatively $88.5 \%$ ( $p=$ 0.002 ) had no urinary loss during sexual activity and 12 months after surgery $93.5 \%(p<0.001)$. Compared to prior to surgery, the satisfaction with current sexual life was increased at 6 weeks $(52 \% ; p=0.048)$ and 12 months $(58 \% ; p=0.029)$. Pain during and after sexual activity was significantly diminished 12 months after surgery $(p=0.019)$.

Discussion: The TOT procedure has a positive influence on female sexual functioning by reducing the urinary leakage and pain during sexual activity. It seems to have a positive effect on the overall sexual satisfaction. Further research is warranted to support these preliminary findings.

\section{FC_16}

Tunnel vision at abdominal hysterectomy; how to broaden the scope towards a laparoscopic approach R. Niewenweg, J.M.A. Pijnenborg, J.F. ter Haar Tweesteden Ziekenhuis, Tilburg, Netherlands

Objective: To identify the choice of surgical route in patients who were planned for a hysterectomy with contraindications to a vaginal approach, as planned by residents, general gynaecologists and laparoscopic-orientated gynaecologists. To determine appropriate criteria for the selection of patients suitable for a laparoscopic hysterectomy.

Materials and methods: A retrospective analysis was performed of all patients for who an abdominal or laparoscopic hysterectomy was planned between January 2005 and April 2008 in a teaching hospital in the South of The Netherlands. Data regarding the surgical route of choice, success of the procedure, conversion of 
laparoscopic assisted vaginal hysterectomy (LAVH) or total laparoscopic hysterectomy (TLH) to abdominal hysterectomy (AH) and feasibility of LAVH or TLH were analyzed and selection criteria used for the laparoscopic hysterectomy were compiled.

Results: A total of 183 hysterectomies were performed during the study period of which 63 (34\%) were planned as LAVH or TLH. Laparoscopic-orientated gynaecologists planned a laparoscopic approach in $77 \%$ of their patients. The percentage of laparoscopic hysterectomies (LH) as planned by residents was 37.5\%, compared to $6 \%$ as planned by general gynaecologists.

$\begin{array}{lll}\text { Residents } & \begin{array}{l}\text { General } \\ \text { gynaecologists }\end{array} & \begin{array}{l}\text { Laparoscopic- } \\ \text { orientated }\end{array} \\ & & \text { gynaecologists }\end{array}$

\begin{tabular}{lllll}
\hline $\begin{array}{l}\text { Laparoscopic } \\
\text { hysterectomy }\end{array}$ & $9(37,5 \%)$ & $6(6 \%)$ & $48(77 \%)$ & \\
$\begin{array}{c}\text { Abdominal } \\
\text { hysterectomy }\end{array}$ & $15(62,5)$ & $91(94 \%)$ & $14(23 \%)$ & \\
\begin{tabular}{l} 
Total \\
\hline
\end{tabular} & 24 & 97 & 62 & 183 \\
\hline
\end{tabular}

Conclusion: The choice of a $\mathrm{LH}$ as alternative to $\mathrm{AH}$ is poorly implemented by general gynaecologists. Residents could play an important role in the change of surgical route in new techniques such as a laparoscopic approach. A decision tree, including important criteria for appropriate selection could be of value in broadening the scope towards a laparoscopic approach.

\section{FC_17}

Follow up after laparoscopic assisted vaginal resection of endometriosis in the septum rectovaginale and the rectosigmoid in women with deep infiltrating endometriosis

\section{A. Kavallaris}

Department of Gynecology and Obstetrics, Friedrich Schiller University Jena, Jena, Germany

Background: Endometriosis with bowel involvement is a most invasive form and can cause infertility, chronic pelvic pain and bowel symptoms. Effective surgical treatment of endometriosis requires complete excision of endometriosis and in same case may require segmental rectosigmoid resection.

Materials and methods: Between 12 of 1,997 and ten of 2,003 55 patients with rectovaginal endometriosis underwent a combined laparoscopic vaginal technique. Thirty patients were found at a follow up and underwent a telephone interview. The questionnaire covered questions about symptoms related to a recurrence of intestinal endometriosis, dyspareunia, dysmenorrhea and pregnancy. Results: Twenty-seven of $30(90 \%)$ women have no clinical symptoms of recurrence of endometriosis. Two patients $(6.6 \%)$ reported recurrence of bowel endometriosis. Dysmenorrhoea disappeared in $28(93.3 \%)$, dyspareunia in $26(86.7 \%)$ and pelvic pain in $27(90 \%)$ patients. Seventeen patients $(31 \%)$ tried to become pregnant and 11 of these patients $(65 \%)$ became pregnant: nine patients delivered healthy newborns, 18 pregnancies were reported and 19 healthy children were born.
Conclusions: Despite the small number of follow up patients, our 94 months follow up data demonstrated that endometriosis with bowel involvement and radical resection was associated with significant reductions in painful and dysfunctional symptoms a low recurrence rate $(6.6 \%)$ and high pregnancy rate $(36.6 \%)$.

\section{FC 18}

A systematic review and meta-analysis of 3,348 women in 39 randomised trials comparing hysterectomy, endometrial ablation and the levonorgestrel-releasing intrauterine system for heavy menstrual bleeding

$\underline{\text { J. Daniels }}^{1}$, R. Champeneria ${ }^{1}$, L. Middleton ${ }^{1}$, N. Hilken ${ }^{1}$, R. Gray ${ }^{1}$, S. Bhattacharya ${ }^{2}$

${ }^{1}$ University of Birmingham, Birmingham, UK, ${ }^{2}$ University of Aberdeen, Aberdeen, UK

Objective: To systematically review and meta-analyse published data from randomised controlled trials of hysterectomy, endometrial ablation and the levonorgestrel releasing intrauterine system (LNG-IUS) in the treatment of heavy menstrual bleeding.

Materials and methods: Electronic bibliographies (MEDLINE, EMBASE, the Cochrane Library, Meta-Register of Controlled Trials) were searched and randomised controlled trials comparing any combinations of the treatments described above were selected. Quality and outcomes were reviewed and data extracted by two independent reviewers. The Cochrane meta-analysis of Lethaby 1999 and 2005 and of Marjoribanks 2006 were updated where possible.

Results: Three thousand three hundred forty-eight women in 39 studies were included in our review. Eight of these studies involving 895 participants were not included in previous Cochrane reviews

\begin{tabular}{llll}
\hline Comparison & Date range & $\begin{array}{l}\text { No. } \\
\text { studies }\end{array}$ & $\begin{array}{l}\text { Total no. } \\
\text { patients }\end{array}$ \\
Hysterectomy vs ablation & $1994-2007$ & 7 & 1,136 \\
Ablation vs ablation & $1992-2006$ & 23 & 1,498 \\
Ablation vs LNG-IUS & $1998-2006$ & 8 & 478 \\
\hline
\end{tabular}

The ability to extract outcome data and update existing meta-analyses was limited by poor quality data reporting. Many studies used unique outcomes or did not present data in a suitable format. For example, in the comparison of endometrial ablation versus hysterectomy, no useful data could be extracted from two new studies. Where updates were possible, the most noteworthy difference to the overall treatment effect in the Cochrane reviews was that the odds ratio for amenorrhoea at 1 year between ablation and LNG-IUS decreased from 1.90 (95\%CI $0.76-4.73)$ to $1.21(0.62-2.38)$.

Conclusions: Failure to report to CONSORT guidelines leads to suboptimal systematic reviews and potentially biased metaanalyses. We are undertaking individual patient data meta-analyses to overcome some of these problems and to allow more reliable indirect comparisons between treatments. We will also construct a decision analytic model to estimate the most cost-effective treatment for particular subgroups of women. 
FC_19

\section{Hysterectomy rates in 1 and 2 years follow up after first consultation in a specialized abnormal vaginal bleeding policlinic \\ K. Hauge \\ University of Tromsø, Tromsø, Norway}

Introduction: In order to evaluate the efficacy of a specialized policlinic for abnormal vaginal bleeding the number of hysterectomies after 1 and 2 years were compared with the suggested treatment plans offered at the end of the first consultation. The aim of a specialized bleeding policlinic is two-fold: to offer a rational treatment plan at the first consultation and secondly to keep the number of major interventions (i.e. hysterectomies) on a minimum for benign conditions.

Materials and methods: One hundred four patients with abnormal vaginal bleeding were examined by the author with TVS, salineinfusion-sonography and endometrial sampling at the first consultation. Focus were held on the possibility to offer minimal-invasive treatment when endometrial sampling was benign and uterus size made such options reasonable. Special attention were paid to intrauterine anatomy in order to properly select those who could profit from transcervical surgery. Treatment options where then discussed and the possibility of preserve fertility was taken in consideration if explicit demanded. The initial treatment proposals and the completed first treatment where compared with the actually undertaken interventions after 1 and 2 years.

Results: Initially only nine hysterectomies were considered necessary, mainly because of enlarged fibroid uteri, the rest could be handled with transcervical surgery, LNG-IUS or medication.(table1) After 1 year the number of hysterectomies raised to 19 and in the 2 year follow up additionally five hysterectomies were undertaken to achieve satisfactory results.

Discussion: In this study almost $25 \%$ ended up with hysterectomy. Abnormal vaginal bleeding is a common problem. This makes the total costs of this intervention considerable. Even if this figure is not high, we have to search for more effective methods to treat this condition without resolving to major surgery.

FC_21

Randomised controlled trial of NovaSure ${ }^{\circledR}$ endometrial ablation and HydroTherm Ablation ${ }^{\circledR}$

J.P.M. Penninx, B.W. Mol, M.Y. Bongers

Maxima Medical Centre, Veldhoven, Netherlands

Introduction: Thermal ablation is a well accepted method in the treatment of menorrhagia. We compared the effectiveness of NovaSure ${ }^{\circledR}$ endometrial ablation and HydroTherm Ablation ${ }^{\circledR}$ (HTA) in the treatment of women with menorrhagia.

Materials and methods: Consecutive women suffering from menorrhagia based on dysfunctional uterine bleeding were asked to participate in a randomised controlled trial. After informed consent, women suffering from menorrhagia were randomly allocated to the NovaSure $^{\circledR}$ procedure or HydroThermAblation ${ }^{\circledR}$. Women were unaware of the type of treatment that had been performed. Patients were asked to complete questionnaires on their bleeding pattern, satisfaction as well as quality of life at 4 weeks, 6 months and 12 months after surgery. The study timed to recruit 160 patients, and was registered under ISRCTN23845359. Primary outcome was patients satisfaction. Moreover, we registered the number of reinterventions. Analysis was done by intention-to-treat. For continuous measures, we used repeated measures analysis of variance.

Results: Between March 2005 and January 2008, we included 161 women, of which 78 were allocated to the NovaSure ${ }^{\circledR}$ group, and 83 to the HTA group. No complications occurred in either of the treatment groups. Patients satisfaction was significantly better after NovaSure ${ }^{\circledR}$ ( $p=0.009$ ) (complete satisfaction at 12 months of $63 \%$ versus $37 \%$ ). Amenorrhoea rates were $40 \%$ versus $26 \%$. On the Short Form 36 questionnaire, there was a significant improvement from treatment in both groups on all dimensions. Women in the NovaSure ${ }^{\circledR}$ group scored significantly better on the dimensions physical functioning.

Conclusion: In the treatment of menorrhagia, NovaSure ${ }^{\circledR}$ endometrial ablation is superior to HydroTherm Ablation ${ }^{\circledR}$

\section{FC_22}

What determines the need to morcellate the uterus during total laparoscopic hysterectomy?

G. Condous $^{\text {I }}$, T. Bignardi ${ }^{1}$, D. Alhamdan ${ }^{1}$, B. Van Calster ${ }^{2}$, S. Van Huffel ${ }^{2}$, A. Lam ${ }^{3}$

${ }^{1}$ University of Sydney-Nepean Centre for Perinatal Care, Sydney, Australia, ${ }^{2}$ Department of Electrical Engineering (ESAT)-KU Leuven, Leuven, Belgium,

${ }^{3}$ Centre for Advanced Reproductive Endosurgery (CARE)-Royal North Shore Hospital-University of Sydney, Sydney, Australia

Objectives: To identify factors associated with the need to perform uterine morcellation during total laparoscopic hysterectomy (TLH). A secondary aim was to establish new cut-offs based on uterine weight for the probability of morcellation.

Materials and methods: Prospective observational study. All women scheduled to undergo TLH from May 2005 to February 2006 were included in the study. Age, parity, operating time, estimated blood loss (EBL), and final uterine weight at histology were recorded. Logistic regression analysis was performed to determine the factors associated with the need to perform uterine morcellation at the time of TLH.

Results: One hundred twelve consecutive women underwent TLH and were included in the final analysis. Fifty-seven of $112(51 \%)$ women underwent TLH without morcellation, i.e. it was possible to deliver the uterine specimen vaginally, and 55 of 112 (49\%) women underwent TLH with morcellation, i.e. it was not possible to deliver the uterine specimen vaginally and therefore uterine morcellation was performed. Median age in the morcellation and no-morcellation group was 46 years (range 36-60 years) and 45 years (range 34 71 years), respectively. There was no significant difference between operating time and EBL between the two groups. Logistic regression analysis demonstrated that uterine weight and parity were significant variables for the prediction of uterine morcellation at the time of TLH. Nulliparity increased the odds of morcellation $[\mathrm{OR}=2.30$ $(0.88-5.96,95 \%$ C.I. $)]$, as too did every $100 \mathrm{~g}$ increase in uterine weight $[\mathrm{OR}=2.33(1.51-3.57,95 \%$ C.I)]. Above a threshold in uterine weight of $350 \mathrm{~g}, 95 \%$ of women required morcellation. 
Discussion: Nulliparity and increasing uterine weight are significantly associated with the need to perform uterine morcellation at the time of TLH. Studies are needed to find a reliable method for estimating uterine weight pre-operatively.

\section{FC 23}

Power Doppler ultrasonography usefulness in selection of endometrial destruction procedures in women with endometrial hyperplasia R. Moszynski, D. Szpurek, M. Englert-Golon, S. Sajdak Division of Gynecological Surgery, University of Medical Sciences, Poznan, Poland

Objective: The aim of this study was to evaluate if Power Doppler ultrasonography may by useful in preoperative qualification for endometrial ablation techniques in group of women with endometrial hyperplasia. It is interesting to check if blood flow parameters vary according to type of endometrial hyperplasia and if they are useful in selection of patients with atypical hyperplasia where small invasive techniques are contraindicated.

Materials and methods: Ninety-six women diagnosed in our clinic with endometrial hyperplasia were examined with Power Doppler transvaginal ultrasonography between 2002 and 2008. Blood flow parameters (PI, RI and TAMXV) were measured in uterine arteries, endometrial and subendometrial vessels and analyzed retrospectively for statistical differences between group of patients with atypical and non-atypical hyperplasia. Results were compared using MannWhitney test with statistical significance $p<0.05$.

Results: Mean age of patients was 56.5 years. There was 54 women with non-atypical endometrial hyperplasia (NAH) and 42 patients with atypical hyperplasia (AH). It was possible to show and analyze blood flow in endometrial or subendometrial arteries in 18 of $54(32.1 \%)$ patients with NAH and 17 of 42 (40.5\%) patients with AH. The mean PI and standard deviation for patients with non-atypical and atypical hyperplasia were $1.38 \pm 0.30$ and $1.39 \pm 0.24$, respectively. The mean RI and standard deviation for patients with non-atypical and atypical hyperplasia were $0.69 \pm 0.12$ and $0.66 \pm 0.11$, respectively. The mean TAMXV and standard deviation for patients with non-atypical and atypical hyperplasia were $15.96 \pm 2.35$ and $17.0 \pm 4.47$, respectively. There was no statistically significant differences in all of presented parameters $p>0.05$. There was also no statistically significant differences between blood flow parameters in uterine arteries $(p>0.05)$.

Conclusions: Because there are no statistically significant differences in blood flow Doppler examination in women with endometrial hyperplasia according to its atypical character this diagnostic method is useless in qualification patients for small invasive endometrial ablation techniques.

\section{FC 24}

Long-term outcome following laparoscopic supracervical hysterectomy and supracervical abdominal hysterectomy

M. Lieng, A. Birte Lømo, O. Istre, E. Qvigstad

Ullevål Universuty Hospital, Oslo, Norway
Objective: Evaluation and comparison of long-term outcome following laparoscopic supracervical hysterectomy (LSH) and supracervical abdominal hysterectomy (SAH).

Design: Retrospective postal questionnaire.

Setting: Norwegian university teaching hospital.

Population: Four hundred seventy-nine consecutive patients.

Materials and methods: A questionnaire was initially sent to all women who underwent LSH during 2004 and 2005. As a consequence of the results, the questionnaire was more recently sent to all women who underwent SAH during 2004 and 2005 as well.

Main outcome measures: Persistent vaginal bleeding and pelvic pain, patient acceptability of such symptoms and patient satisfaction following LSH and SAH.

Results: Among women who underwent LSH, 78\% completed the questionnaire. Twenty-four percent reported experiencing vaginal bleeding up to 3 years following the LSH, although this was rated as minimal in $90 \%$ of cases, resulting in a mean bothersome score of 1.1 (SD 2.0) on a ten-point visual analogue scale (VAS). $74 \%$ of women reported having menstrual pain prior to the LSH, with a mean score of 6.8 (SD 2.1) (10-point VAS). Up to 3 years after the LSH, 38\% continued to experience menstrual pain, although this was significantly less intense with a mean score of 3.5 (SD 2.2) ( $p$ $<0.01$ ). Ninety percent of women reported being satisfied with the LSH. Among women who underwent SAH, 75\% completed the questionnaire. Preliminary analyses indicate a lower frequency of persistent bleeding following SAH compared to after LSH. The long-term outcome following SAH will be presented, and the outcomes after LSH and SAH will be compared.

Conclusion: Although vaginal bleeding and pelvic pain are frequently observed following $\mathrm{LSH}$, these symptoms are significantly reduced and patient satisfaction is high. The corresponding results following SAH will be presented.

\section{FC 25}

Two thousands forty nine outpatient clinic hysteroscopy sterilizations. Patient satisfaction and tolerance

J. Eduardo Arjona, B. Povedano, B. Pelegrin, I. Jeronimo, E. Sainz de Vicuña, B. Hinojosa, E. Velasco

Reina Sofia Hospital, Córdoba, Spain

Introduction: The Essure ${ }^{\circledR}$ microinsert system (Conceptus, inc., Mountain View, CA) is a modern device used for tubal sterilization. In an outpatient clinic the device is placed transcervically via hysteroscopy catheterization. We evaluated the rate of successful insertion, tolerance and women's satisfaction. No anaesthesia or sedation was necessary.

Materials and methods: A prospective analysis of case series was design. A total number of 2,849 women underwent hysteroscopy Essure $^{\circledR}$ sterilization since 2003 to 2007. After 3 months, satisfaction was assessed by visual analog scale. Adverse effects and tolerance were also recorded.

Results: The rate of successful insertion was $99 \%$ (2,821 cases). The same day of insertion $75 \%$ women returned to their daily activities. Eighty-six percent patients considered the procedure painless or scarcely painful. All the patients were highly satisfied $(89.2 \%)$ or satisfied $(10.7 \%$ ). Ninety-four percent rated the method 10 or 9 (on 0 
to 10 scale). The most valuables aspects of the procedure were absence of surgery (42\%), technique quick and comfortable (28\%), and permanent sterilization (20\%). Of the patients, $99.8 \%$ have already recommended or would recommended the procedure.

Discussion: Hysteroscopy Essure ${ }^{\circledR}$ tubal sterilization can be placed in an outpatient clinic without anaesthesia or sedation. Patients report high tolerance and describe minor postoperative pain. This technique is cost-effective and would be consider as the first choice for women who desire a sterilization procedure.

\section{FC_26}

\section{Complications associated with Essure ${ }^{\circledR:}$ an analysis of 2,849 performed cases}

J.E. Arjona Berral, E. Sainz de Vicuña Sainz, E. Velasco Sánchez, I. Jerónimo Franco, B. Povedano Cañizares

Hospital Universitario Reina Sofia, Córdoba, Spain

Objective(s): Description of the complications registered in our reference population.

Materials and methods: Analysis of 2,849 patients of our unit, in which we have placed the tubal microinserts.

Results: The complication rate is $1.05 \%$. From them, the most frequent is the insert expulsion (14 cases). We have registered four descent, two intramiometrial placement, and two migrations. In four cases we have diagnosed pregnancy after the placement, three because of device failure (1 ectopic pregnancy). We have only registered one infectious complication (endometritis).

Conclusions: The Essure device is a safe method, not only as a contraception method but also because of the low number of complications, being the most frequent the expulsion of inserts.

\section{FC_27}

\section{Relationship between preoperative symptoms} and extent of disease: comparison of a new classification system for endometriosis with the revised American Society for Reproductive Medicine classification

C. Gulumser, S. Kayani, E. Saridogan, A. Cutner

University College London Hospital, London, UK

Objective: To determine if better relationship between preoperative symptoms and extent of disease can be established using a new classification system using SF-36 scores, VAS (Visual Analogue Scale) and SF-MPQ (Short-Form McGill Pain Questionnaire).

Materials and methods: Prospective cohort study. Between April 2001 and Oct 2007 all women attending our endometriosis clinic were asked to complete the above questionnaires. 191 women filled the questionnaires pre- and postoperatively. Preoperative symptom scores were compared against the operative findings to identify any correlation between rASRM and a new classification formulated by our unit.

Results: We classified the operative findings into five groups of superficial endometriosis, deep sidewall disease, endometrioma, rectovaginal disease + endometrioma and deep sidewall disease + endometrioma which comprised of 54(28\%), 61(31\%), 13(6\%), 19 $(22 \%), 43(22 \%)$ subjects in each group respectively. No signifi- cant correlation was found between the preoperative questionnaire scores and the rASRM score (Spearman's rho $p>0.05$ ). In addition, no significant associations was observed between severity of the disease according rASRM stage or our new classification categories (Kruskal Wallis Test, $d f=3, p>0.05$ ) and the preoperative questionnaires. We also analysed the degree of correlation between anatomical localizations of disease and pain types (Kendall's tau-b). The most significant correlations were those of dyspareunia, dysmenorrhoea and dyschesia where the disease location was uterosacral ligament $(p=0.001)$, right uterosacral ligament $(p=0.009)$ and pararectal area $(p=0.003)$ respectively.

Conclusions: There appears to be no relationship between the rASRM or our surgical classification and the preoperative QoL scores. The widely accepted rASRM classification has an inadequate relationship with symptoms of endometriosis. We have demonstrated statistically significant correlations between the symptoms and disease site. There is a clear need to formulate a system or tool which would effectively correlate the patient indicators, whether assessed as symptoms or quality of life assessment or a combination, with operative findings. This would provide an ideal mechanism of assessment which would enable clinicians to improve their diagnostic precision and consequently plan for an appropriate surgery.

\section{FC_28}

Laparoscopic anatomical nerve sparing radical hysterectomy for cervical cancer: technique and results Z. Liang, Y. Chen, H. Xu, Y. Li, D. Wang, J. Li, J. Yuan Department of Obstetrics and Gynecology, Southwest Hospital, Third Military Medical University, Chongqing, China

Objectives: To investigate feasibility of laparoscopic anatomical nerve sparing radical hysterectomy and the impact on early bladder function, we introduce a space anatomic method and meticulously separated the blood vessels and connective tissues to preserve the pelvic splanchnic nerve, the hypogastric nerve, and the bladder branch of the inferior hypogastric plexus under magnification $(\times 10.5)$ during laparoscopic radical hysterectomy with pelvic lymphadenectomy.

Materials and methods: Thirty-seven patients (FIGO stage IB1, $n=12$, stage IB2, $n=10$, and stage IIA, $n=15$ ) underwent laparoscopic anatomical nerve sparing type III radical hysterectomy with pelvic lymphadenectomy for cervical cancer during 2006 to 2007. Postoperative assessment of bladder function include: the time to recovered the ability to void her bladder spontaneously and achieve a postvoid residual urine (PVR) volume less than $50 \mathrm{ml}$. Results: The laparoscopic anatomic nerve-sparing procedure was completed successfully and safely in all of the patients. The mean operating time was 159 min (range: 130-228). The mean blood loss was $189 \mathrm{ml}$ (range: 100-300). Eight (36\%) and six (27\%) patients had the PVR of $<100 \mathrm{ml}$ and $<50 \mathrm{ml}$ respectively at the initial removal of the catheter after operation 5 days. On the fourteenth day, $82 \%$ and $77 \%$ of the patients had the PVR of $<100 \mathrm{ml}$ and $<50 \mathrm{ml}$, respectively. The mean duration before the PVR became $<50 \mathrm{ml}$ was 11.27 (5-26) days. 
Conclusions: The technique described in this preliminary study appears safe, adequate, and feasible in our population with satisfactory recovery of voiding function. Further prospective controlled studies are needed to confirm our data.

\section{FC_29}

Prognostic value of microvessels densities in patients with endometrial hyperplasia and adenocarcinoma

V. Bourlev, S. Sarkisov, E. Dubinskaya

Scientific Center For Obstetrics, Gynecology and Perinatology, Moscow, Russian Federation

The aim of the study was to assess microvessel density (MVD) and diameter of blood vessels $(<2 \mu \mathrm{m}$ or $>2 \mu \mathrm{m})$ as a marker for angiogenesis in endometrial hyperplasia, adenocarcinoma and normal endometrium, and to determine its prognostic value.

Materials and methods: Fifty-two patients were included in the study. According to WHO-classification all the patients were divided in to four groups: $1-$ control group (19 patients), 2patients with non-atypical hyperplasia (simplex-10, complex7), 3-patients with atypical hyperplasia (simplex - 5, complex4), 4-patients with adenocarcinoma (7). The diagnosis was confirmed histologically. Assessment of microvessel density was performed using histopathological immunoperoxidase methods, using an anti-von Willebrand factor antibody (DAKO A/S). The diameter of blood vessels was measured using transmission electron microscopy. The vessels $<2 \mu \mathrm{m}$ in diameter were called "shoots".

Results: The MVD was significantly higher in patients with atypical hyperplasia and adenocarcinoma compared to values of the control group and in patients with non-atypical hyperplasia. We evaluated the vessels $(>2 \mu \mathrm{m}) /$ "shoots" $(<2 \mu \mathrm{m})$ ratio. In patients of the controls and in patients with non-atypical hyperplasia this ration was $11.4 \pm 0.7$. This correlation coefficient increased significantly in patients with atypical hyperplasia and adenocarcinoma due to the reduction of the "shoots" number.

Conclusion: MVD and microvessels/"shoots" ratio could be useful significant prognostic criteria and could be helpful in identification of patients with endometrial pathology at high risk of malignancy.

\section{FC 30}

\section{Impact of surgical peritoneal environment on postoperative dissemination and tumor growth in a preimplanted tumor model \\ A.-S. Azuar, S. Matsuzaki, N. Bourdel, J.-L. Pouly, G. Mage, M. Canis \\ CHU Clermont-Ferrand, Polyclinique, Clermont-Ferrand, France}

Objective: We recently demonstrated that surgical peritoneal environment of $\mathrm{CO} 2$ pneumoperitoneum at a low intraperitoneal pressure (IPP) could be optimal to minimize peritoneal dissemination, when tumor cells were inoculated just before surgery, in a syngenic mouse model. However, to mimic a clinical setting, a different study design in which tumors are present before surgery is also necessary. The objective of the present study was to evaluate the impact of surgical peritoneal environment on postoperative dissemination and tumor growth over time in a preimplanted tumor model. We used our established mouse surgical model with controlled respiratory support.

Materials and methods: On day -7, C57BJ6 mice received an intraperitoneal inoculation of a mouse ovarian cancer cell line (ID8). On day 0 , mice were randomized into four groups of 32 animals each: anesthesia alone, $\mathrm{CO} 2$ pneumoperitoneum at low $(2 \mathrm{mmHg})$ or high $(8 \mathrm{mmHg})$ IPP and laparotomy. Groups were further subdivided into four groups and a laparotomy was performed to evaluate dissemination and tumor growth on post-operative day (POD) 1, 2, 7 or 14. Comparisons were made using the one-way ANOVA.

Results: The peritoneal dissemination score was significantly higher in the laparotomy group compared to the remaining three groups on PODs 1,2 and 7. We detected no significant difference in the peritoneal dissemination score among the low, high IPP and anesthesia groups on PODs 1, 2 and 7. However, there was no significant difference in the peritoneal dissemination score among the three surgical groups on POD 14. We detected no significant difference in the diaphragmatic dissemination score among the four groups on PODs 1, 2 and 7, whereas it was significantly higher in the three surgical groups than the anesthesia group on POD 14. Histopathological examination demonstrated that the incidence of invasion of cancer cells into muscle layers was significantly higher in the laparotomy group than the low IPP and anesthesia groups on POD 14.There was no significant difference in tumor growth among the four groups.

Conclusions: Our previous and the present findings suggested that a $\mathrm{CO} 2$ pneumoperitoneum at a low IPP had few if any short term effects on peritoneal dissemination in either a tumor cells spillage or preimplanted tumor model.

\section{FC 31}

The accuracy of risk scores in predicting ovarian malignancy; a systematic review

P. Geomini ${ }^{1}$, R. Kruitwagen ${ }^{2}$, G. Bremer ${ }^{3}$, J. Cnossen ${ }^{4}$,

B. Willem $\mathrm{Mol}^{4}$

${ }^{1}$ Maxima Medical Centre, Veldhoven, Netherlands, ${ }^{2}$ University Hospital Maastricht, Maastricht, Netherlands, ${ }^{3}$ Maasland Hospital Sittard, Sittard, Netherlands, ${ }^{4}$ Academic Medical Centre, Amsterdam, Netherlands

Introduction: Preoperative assessment of the potential malignancy of an ovarian mass is important for optimal surgical treatment. The risk of malignancy of ovarian masses can be assessed preoperatively with prediction models, in which multiple factors are included. The purpose of the present study was to perform a systematic review of the literature on the accuracy of prediction models in the pre-operative assessment of the adnexal mass.

Materials and methods: Studies were identified through the Medline databases, Embase, Cochrane library and Medion from 1966 to March 2008. Language restrictions were not applied. Two independent reviewers selected studies and extracted study characteristics, study quality and test accuracy. Accuracy data were used to form $2 \times 2$ contingency tables of the results of the risk score versus 
definitive histology. We used bivariate meta-analysis to estimate pooled sensitivities and specificities with $95 \%$ confidence intervals as well as summary Receiver Operating Characteristic (sROC) curves.

Results: There were 83 accuracy studies reporting on 17,796 adnexal mass (4,983 malignancies), that met the selection criteria. These studies reported on 63 different prediction models, one of which the model developed by Sassone was the most evaluated. All described prediction models have acceptable sensitivity en specificity. However, the Risk of Malignancy Index (RMI) I and II appeared to be the best predictors.

Conclusion: Based on our review, the RMI should be the test of choice in the pre-operative assessment of the adnexal mass.

\section{FC_32}

Micro-laparoscopic management of endometrial cancer G. Siesto, A. Cromi, F. Zefiro, P. Beretta, B. Elena, F. Ghezzi

Department of Obstetrics and Gynecology, University of Insubria, Varese, Italy

Objective: The aim of this study was to evaluate the feasibility and safety of the surgical management of endometrial cancer with a micro-laparoscopic approach.

Materials and methods: Between October 2007 and May 2008 consecutive patients addressed to surgery for the treatment of apparently early stage endometrial cancer were enrolled in this study. Elevated BMI was not considered as a contraindication to micro-laparoscopy. The micro-laparoscopic procedures were carried out with 3-mm instruments introduced through three ancillary port sites and a 5-mm umbilical telescope. Surgical staging included peritoneal cytology, total laparoscopic hysterectomy with bilateral salpingo-oophorectomy and bilateral systematic pelvic lymphadenectomy. Data obtained were than compared to those of the last 50 patients treated for endometrial cancer with conventional laparoscopic approach. Operative time, blood loss, lymph nodes count and hospitalization were considered as primary end-points.

Results: During the study period eight patients [age 63 years (range 54-77)] were prospectively enrolled. Mean BMI was $28.0 \pm 6.2 \mathrm{~kg} / \mathrm{m}^{2}$. The mean operative time required for the procedure was $160 \pm 30.9$ min with an estimated blood loss of 110 (range 10-200) $\mathrm{mL}$. Average number of lymph nodes removed was 17.9 (range 11-29). Patients were discharged 2 (range 1-4) days after surgery. No major intra-operative or postoperative complications occurred. No conversion to laparotomy was needed. No difference was found in terms of operative time, estimated blood loss, lymph node yield removed between patients undergoing microlaparoscopy and the control group. All patients who underwent microlaparoscopic treatment are currently under follow up and resulted NED at the last visit.

Conclusions: Micro-laparoscopy in skilled surgical teams can be successful applied for the performance of pelvic lymphadenectomy and it can represent a viable route for the management of endometrial cancer. Larger series and longer follow-up are obviously needed to establish its oncological safety.
FC_33

Laparoscopy versus laparotomy in the treatment of early stage endometrial cancer patients: a randomised multi centre cost-effectiveness tria

$\underline{\text { C. Bijen }}^{1}$, G. de Bock ${ }^{2}$, J. Keurentjes ${ }^{1}$, L. Ulkeman ${ }^{1}$, M. Mourits ${ }^{1}$

${ }^{I}$ Department of gynecology, University Medical Centre Groningen, Groningen/Groningen, Netherlands,

${ }^{2}$ Department of epidemiology, University Medical Centre Groningen, Groningen/Groningen, Netherlands

Objective: Traditionally standard treatment for patients with early stage endometrial cancer (EC) is total abdominal hysterectomy and bilateral salpingo oophorectomy (TAH + BSO) through a vertical midline incision. While TAH is an accepted effective treatment, it is highly invasive, visibly scarring and associated with adverse events such as blood loss and wound problems. Morbidity of laparotomy in case of endometrial cancer is substantial (43\%) due to frequent obesity and co-morbidity in this patient group and hospital stay is usually at least 1 week. Though in several studies total laparoscopic hysterectomy (TLH + BSO) seems a safe and feasible alternative approach in early stage endometrial cancer patients, there are no randomised data on laparoscopy in these patients applicable to the Dutch situation. The aim of this study is to compare the treatment related morbidity and cost-effectiveness in early stage endometrial cancer patients treated by laparoscopy versus the standard approach by laparotomy. Materials and methods: A multicentre randomised clinical phase 3 trial, including five university hospitals and 15 regional hospitals in the Netherlands. Only gynaecologist with a sufficient OSATS (Objective Structured Assessment of Technical Skills) score ( $\geq 28$ points) are allowed to participate. An independent clinical review board will assess and judge the severity of all recorded complications. In 30 month, 275 patients will be randomised (2:1) to laparoscopy or laparotomy. The main outcome measure is the rate of major complications, being an indicator of clinically relevant treatmentrelated morbidity. Secondary outcome measures are (1) costs and cost-effectiveness, (2) minor complications, (3) quality of life.

Results: Until now, 27 gynaecologists in 18 centres participate, having sufficient laparoscopic skills in performing a TLH according to an OSATS $\geq 28$ points. The average time to initiate the study in a new participating centre is 5.5 months. Currently, 156 patients are enrolled. Due to protocol violation two patients were excluded from further analysis. Two centres were banned for further participation because of selection bias: patients were offered the experimental surgical procedure outside the study.

Conclusion: A randomised multi center study in early stage endometrial cancer patients with inclusion criteria for patients and surgeons is being conducted. We expect to complete inclusion by the end of 2009 .

FC_34

Laparoscopically assisted radical vaginal hysterectomy on patients with cervical and endometrial cancer-3-year experience

G. Gorchev, S. Tomov, L. Tanchev, A. Velkova

Medical University, Pleven, Bulgaria 
Objective: The authors present their 3-year experience in the field of laparoscopic treatment of cervical cancer and endometrial cancer. Materials and methods: For the period January 2005-December 200749 female patients with invasive cervical cancer (T1а2-Т1в) and three female patients with invasive endometrial cancer (Т1в) have been subject to laparoscopically assisted radical vaginal hysterectomy with pelvic lymph node dissection.

Results: The mean age of patients was $43.1( \pm 10.1)$ years as the dominant age group was within the range of $40-49$ years $(n=20$; $38.5 \%)$. The mean operative time was 193.73 min $( \pm 77.8)$. Significant difference has been found in the values of the preoperative and post-operative hemoglobin (on the first postoperative day), respectively 126.94 and $116.44 \mathrm{~g} / 1(p=0.0001)$. The mean number of removed lymph nodes was $9.76( \pm 5.04)$ and lymph metastases (N1) have been found in five of the cases (9.6\%). Complications have been observed in two of the patients (3.8\%): one with a hemorrhage from the vaginal cuff (on the first post-operative day) and one with a pelveocellulitis (on the fifth post-operative day).

Conclusion: Laparoscopic method of the treatment of cervical and endometrial cancer represents a safe alternative to the open abdominal surgery in patients at the first clinical stage. The surgical intervention should be performed by gynecologists experienced in the oncologic and laparoscopic surgery.

\section{FC_35}

Laparoscopic surgery in management of adnexal tumors-unexpected malignancy

T. Guerra, E. Suarez, L. Mañalic

University Hosp. Vall de Hebron, Barcelona, Spain

Objective: To assess the efficacy and safety of laparoscopy sugary in the management of adnexal tumors with no sings of malignancy.

Design: Retrospective study.

Materials and methods: A total of 2.209 adnexal tumors were removed by laparoscopy from 1.971 women. The mean age was 38 years (range: $17-72$ years).

Results: The procedures performed were total cystectomy, and ovariectomy or adnexectomy in those beyond menopause. Preoperative assessment was the same as for as conventional surgery. Transvaginal ultrasonography was performed to evaluate the size and internal characteristics of masses to exclude malignancy, also was evaluate the IR by Doppler-colour. Serum CA 125 and CA 19.9 level was measured in all women at disagnóstic laparoscopy, visual inspection, cytologic examination, and if necessary, biopsy and frozen section were performed. If cytology of the frozen section indicated malignancy, the procedure was converted to laparotomy. Twenty seven patients $(1.22 \%)$ required conversion to laparotomy because of unexpected malignancy, and others 17 patients $(0.86 \%)$ required laparotomy because of complications during or post-surgery.

Conclusions: Laparoscopic management of adnexal tumors is a safe and beneficial method in selected patients when are performed by experienced laparoscopic surgeons. The approach to complex ovarian masses is possible in most patients, however, it should be performed only in centers where an oncologic back-up is immediately available.

\section{FC_36}

\section{Laparoscopic management of adnexal masses} in pregnancy

D. Tutunaru $^{1}$, D. Florentina Lebit ${ }^{1}$, I. Luiza Condulescu ${ }^{1}$, M. Cristian Dumitrascu ${ }^{2}$, B. Alexandru ${ }^{1}$

${ }^{1}$ Eias Emergency Hospital, Bucharest, Romania, ${ }^{2}$

University Emergency Hospital, Bucharest, Romania

Aim: to assess the efficacy and safety of minimal invasive approach of adnexal masses during first and early midtrimester pregnancy

Materials and methods: prospective, nonrandomised study, carried in our clinic between 2004 and 2007. Inclusion criteria: pregnant women with symptomatic large adnexal masses confirmed by ultrasound. Exclusion criteria: pregnancy over 20th week of gestation, history of at least two miscarriages, adnexal masses. From 32 patients enrolled in the study 22 were in the first trimester. Laparoscopy was done under general anesthesia using fentanyl, thiopental, atracurium and sevoflurane. Incision points were done according to uterine size and adnexal masses localisation.

Results: We have found 19 cases of ovarian serous cysts, eight cases of endometriotic cysts, three cases of dermoid cysts, one case of torsion of ovary and one case of retroperitoneal serous cyst. In 13 cases there was bilateral involvement of ovaries. All serous cysts were removed through stripping. All the endometriotic cysts were removed successfully except one whose wall was left in place after drainage and afterwards electrocoagulated. The ovarian torsion was mild and resolved without consequences. The retroperitoneal cyst which involved right broad ligament and extended to round ligament was opened and drained. All dermoid cysts were opened, drained and excised using an endobag as protection. All cases experienced no immediate or late complications. All patients delivered at term healthy babies except two cases of PROM at 35 and 36 weeks.

Conclusions: laparoscopic approach of symptomatic adnexal masses during pregnancy is extremely safe both for the mother and the fetus and offers, beside cure of ailment and removal of symptoms, a sense of safety and mental relief for the mother.

\section{FC_37}

\section{An audit of a nurse led hysteroscopy service D. Holloway Guys and St Thomas NHS foundation Trust, London, UK}

Introduction: This prospective audit describes 8 months of date collected from a nurse led service from January 08 to August 08 . Materials and methods: A prospective audit over 8 months of all women who are seen within the nurse led outpatient clinic. Information is collected on: age, referral source, and indication for referral, findings at hysteroscopy, management plan and on going care. Comparison for accuracy of diagnosis is also made for women in relation to histology results and comparison of outpatient findings and those at subsequent surgical procedures. 
Results: Data collection is still ongoing but to date there are 79 women who have had hysteroscopy. $58 \%$ of women have been referred from Primary Care. There has been a 7.5\% failure rate (six women). Forty percent of women have been referred for irregular vaginal bleeding, $20 \%$ with menorrhagia and $11 \%$ after an abnormal vaginal ultrasound. Initial findings are 35\% have been referred for further surgery. Sixty-five percent have been investigated and reassured or had outpatient treatment.

Discussion: Initial results show that a nurse led service is a safe and effective front line service in the outpatient setting and can reduce waiting times and increase the number of women who are seen within the current 18 week waiting time.

\section{FC 38}

The development of a clinic for women with inherited bleeding disorders and gynaecology problems

D Holloway, B Madan

Guys and St Thomas NHS foundation Trust, London, UK

Introduction: this is intended to show the development of a clinic that is inter professional/department to ensure seamless care for women with complex haematological and gynaecology problems Materials and methods: a description of the service and its development from the initial pilot to a full service running twice a month.

Results: this clinic has resulted in a decrease in hospital visits for women, increase in knowledge for staff and patients. The clinic provides a holistic service for women within the one visit and allows complex patients to have a comprehensive management plan tailored to their needs. The early intervention in women with bleeding disorders has allowed a better quality of life in relation to decreasing menstrual loss, increasing haemoglobin and decreasing use of blood products. It allows full cover and planning of any interventional procedures. The clinic also sees women who have had a history of thrombosis and need advise about hormone use. Discussion: The clinic has been a success with all slots fully utilised. Patients can be seen in the clinic and have an assessment and review for both of their problems. Complex patients can be managed in this clinic with liaison with medical consultants within gynaecology if needed. This is the only joint clinic within the UK that is staffed by a medical and nurse consultant.

\section{FC 39}

Endometrial preparation for microwave endometrial ablation using progesterone withdrawal: a survey of outcome and satisfaction

G. Steele, D. Rowlands

Arrowe Park Hospital, Upton, Wirral, Merseyside, UK

Introduction: In December 2007, at Arrowe Park Hospital, Upton, Wirral, Merseyside, England, endometrial preparation for M.E.A. (microwave endometrial ablation) was changed from using GnRH analogue injections to using progesterone to induce a withdrawal bleed. This change in practice was introduced as a cost saving exercise in line with N.I.C.E. (National Institute for
Health and Clinical Excellence) guidelines for heavy menstrual bleeding recommendations. Following this change of practice, menstrual outcome and patient satisfaction compared with treatment following $\mathrm{GnRH}$ analogue preparation has not yet been established.

Materials and methods: This retrospective audit using patient case notes and patient questionnaires will assess menstrual outcome and satisfaction at 6 months following progesterone withdrawal preparation compared to $\mathrm{GnRH}$ preparation. It will include all patients who underwent M.E.A. during December 2007, January and February 2008 and will be compared to women who underwent M.E.A. over a 3 month period during September, October and November 2007.

Results: The results will indicate if there is a loss in satisfaction with treatment or menstrual outcome following this change of endometrial preparation.

Discussion: This audit forms an initial review and will be repeated at 12 months and in the longer term to gain an indication of effectiveness, satisfaction and the incidence of any further surgical procedures.

\section{FC_40}

\section{Cavaterm ${ }^{\circledR}$ thermal balloon ablation} of the endometrium

N. Holvey

County Hospital Lincoln, Lincoln, UK

Assessment through audit for Cavaterm ${ }^{\circledR}$ thermal balloon ablation of the endometrium, carried out within the outpatient clinic. Assessment of Cavaterm ${ }^{\circledR}$ using guidelines and protocols for women with dysfunctional uterine bleeding.

Audit carried out to assess: Satisfaction rates 6 months post treatment. Safety and effectiveness of equipment. Effectiveness of pain protocol guidelines.

Results: High satisfaction achieved of $87 \%$. Guidelines set up showed an effective pathway.

Conclusion: This treatment is safe and effective achieving high satisfaction rates.

Discussion: If carried out within the outpatient setting this is a cost effective treatment. The simplicity and safety of this equipment indicates that nurse hysteroscopists and junior doctors could be trained to carry out this technique.

\section{FC_41}

\section{Rectal stenosis after laparoscopic excision of rectovaginal endometriosis N. Waters, G. Maytham, T. Rockall, A. Kent MATTU, Surrey, UK}

Introduction: Endometriosis affects between 4\% and 20\% of the women with chronic pelvic pain. Colorectal involvement is estimated to occur in between $4 \%$ and $37 \%$ of cases, and surgical strategy is not always a clear-cut. We looked at the association between development of postoperative stenosis and presence of serosal fibrosis in the margins of the surgical specimen on 
histology. In addition, a comparison is made with the results from anterior resections performed by the same colorectal surgeon for non-gynaecological diagnoses.

Materials and methods: A database was established for all patients undergoing laparoscopic excision of rectovaginal endometriosis. Outcomes analyzed include operation performed, transfusion requirement, conversion and complication rates, and length of hospital stay.

Results: Between April 2004 and November 2007, 60 patients underwent laparoscopic excision of rectovaginal endometriosis. Thirty-three percent underwent a rectal wall shaving, $13 \%$ had a disc excision and primary closure of the rectal wall, and 55\% underwent an anterior resection. Two percent of patients required a post-operative blood transfusion and there was a conversion rate of $3 \%$. Eleven percent of patients had major complications, including anastomotic dehiscence (5\%), rectovaginal fistulae (2\%), ureteric injury (3\%), and the development of a pelvic abscess (2\%). Five percent of patients required repeat operation, with 3\% necessitating the formation of a temporary stoma. The median duration of hospital stay was 3 days. Of the patients undergoing anterior resection, $18 \%$ later developed anastomotic stenosis requiring dilatation. In patients with clinical signs of stenosis it was noted that either microscopic endometriosis or serosal fibrosis on histology was present more commonly in the resection margins, suggesting a relationship. However, these results show complication rates significantly higher than those seen in anterior resections performed for non-gynaecological diagnoses by the same colorectal surgeon.

Conclusions: There is an association between fibrosis and endometriosis on histology and developing postoperative complications after excision of recto-vaginal endometriosis. Patients should be made aware of the risk of potentially severe complications, and the surgery should only be undertaken by teams highly experienced with this condition.

\section{FC 42}

\section{Management of endometriosis - comments on recurrence and fertility \\ H. Salem, S. Azazi \\ Alex.Univ., Alexandria, Egypt}

Endometriosis is one of greate enigmas in our practice. We face many dark spots in our knowledge about endometriosis. Apart from the mysterious aetiology, pathology, pathophysiology, clinical picture and even the choice of the best line of treatment. In this paper I am going to concentrate on only two important points about endometriosis. The first is the recurrence rates after different lines of surgical treatment. The second is the fertility outcome after laparoscopic management of endometriosis.

Regarding the first item which is the recurrence rates after different types of surgery done in cases of ovarian endometriosis and its comparison with the international figures, we have done a retrospective study on my personal cases done in the last few years and followed up very closely.

The fertility outcome after laparoscopic surgery for endometriosis is a very controversial issue. Also a retrospective search in my personal cases will give us real figures of the problem.

\section{FC 43}

Can different dimensions of pelvic pain help

in the diagnosis of endometriosis?

K. Ballard, S. Banerjee, H. Lane, J. Wright

University of Surrey, Guildford, UK

Introduction: Pelvic pain is a key symptom of endometriosis but also features in other conditions. This limits its diagnostic value, and consequently around $1 / 3$ of women with pelvic pain are found to have no discernable pathology. This study attempts to refine women's reported experiences of pelvic pain by measuring different dimensions and determining their value in the diagnosis of endometriosis.

Materials and methods: Prior to surgery, 185 women undergoing laparoscopy for chronic pelvic pain were asked to complete detailed questions about the descriptions, area, and intensity of pain. At laparoscopy, the surgeon mapped the pathology on a pelvic diagram. Histologically confirmed endometriosis was classified as 'Deep infiltrating Endometriosis' $(>5 \mathrm{~mm})$ or 'Superficial' ( $<5 \mathrm{~mm})$.

Results: One hundred thirteen (61\%) women had histologically confirmed endometriosis, of which $72(64 \%)$ had deep disease and 41 (36\%) had superficial disease. 36 (19.5\%) women had nonendometriosis pathology, and $36(19.5 \%)$ had no identifiable pathology. Three pain descriptors; 'Gnawing', 'Dragging pain to the legs' and 'Throbbing' were reported more frequently by women with endometriosis when compared to women without any identifiable pathology $(p<0.05)$. Adjusted odds ratios revealed that only 'throbbing' was predictive of endometriosis (OR 4.3 $[95 \% \mathrm{CI}=1.6,12.0])$. Individual areas of pain were found to be unrelated to the overall surgical diagnosis and pain in one area did not relate to endometriotic disease in the same pelvic location. Overall pain intensity did not relate to a diagnosis of endometriosis or to having deep or superficial disease. Dyschezia was greater in women with endometriosis compared to those without $(p=0.001)$ and greater in women with deep disease compared with superficial disease $(p=0.023)$.

Discussion: Although area of pain and overall pain intensity do not appear to be useful in differentiating between endometriosis and other causes of pain, specific pain descriptors and the intensity of dyschezia appear to be useful predictors of a diagnosis of endometriosis.

\section{FC_44}

\section{Differences in histologic components among women with recurrent deep infiltrating endometriosis \\ F. De Cicco, P. Carfagna, G. Carri, C. De Cicco, A. Caruso Università Cattolica del Sacro Cuore, Rome, Italy}

Introduction: Rectovaginal endometriosis presents as a nodular lesion composed of fibrotic and endometriotic tissue. In the severe stage, the major component of the lesion is the fibromuscular tissue, that consists of fibrosis and smooth muscle, rather than ephithelial endometriotic tissue. The smooth muscle originates from a metaplastic phenomenon correlated with the increase of 
fibrosis. The aim of this study was to evaluate differences in fibromuscolar metaplasia between women with primary laparoscopic excision of deep infiltrating endometriosis and women presenting a recurrence of disease.

Materials and methods: In 2003-2007, 98 women with rectovaginal endometriotic lesions required laparoscopic excision. The nodules were histologically evaluated and, in addition, we calculated the ratio between fibrosis and entire tissue area: grade (G) 0, no fibrosis; G1<25\%; G2 25-49\%; G3 50-75\%; G4>75\%. During the 60 months follow up, a second laparoscopic excision was performed in nine women with diagnosis of recurrent rectovaginal endometriosis. The lesions were histologically evaluated, and the fibrosis grading was calculated too.

Results: After the first laparoscopic excision among 100 lesions, we found this fibrosis grading distribution: G0 0\%; G1 2\%; G2 3\%; G3 45\%; G4 50\%. After the second laparoscopic excision, among nine lesions we found $\mathrm{G} 0$ in $0 \%, \mathrm{G} 1$ in $0 \%, \mathrm{G} 2$ in $22 \%, \mathrm{G} 3$ in $44 \%$ and $\mathrm{G} 4$ in $36 \%$.

Discussion: These findings show differences in the fibrosis distribution between primary and recurrent lesions. The histological evaluation shows a lower grade of fibrosis in woman who underwent a laparoscopy for recurrence of disease. The metaplastic phenomenon that leads to the formation of fibromuscolar tissue from the smooth muscle could be related to the severity and the age of the lesion. This findings might be of help in differentiate between persistence or recurrence of endometriosis. Although further studies are needed to confirm these data, this is the first study that report a histologic difference in recurrent endometriotic lesions.

\section{FC_45}

\section{Qualitative comparison of postoperative outcomes at 3 and 6 months following laparoscopic surgery for endometriosis amongst two groups of women with good and poor symptomatic correlation with the revised American Society for Reproductive Medicine classification score \\ C. Gulumser, S. Kayani, E. Saridogan, A. Cutner \\ University College London Hospital, London, UK}

Objective: To determine if surgical outcomes differ in two groups of women who have good correlation of their symptoms with the extent of disease and those who have poor correlation using SF36, VAS (Visual Analogue Scale), SF-MPQ (Short-Form McGill Pain Questionnaire) scores at 3 and 6 months following laparoscopic surgery for endometriosis.

Design: Prospective cohort study.

Materials and methods: Between April 2001 and September 2007 all women undergoing planned laparoscopic treatment for endometriosis were asked to complete the above questionnaires. Of these, a complete dataset was attained for 191 women who filled out questionnaires pre-operatively as well as 3 and 6 months postoperatively. Data were analysed using two groups based on level of symptomatic correlation with the rASRM: group 1(good correlation) and group 2 (poor correlation). Post operative QoL assessments at 3 and 6 months were compared.

Results: The mean rASRM score was $(41.16 \pm 43.55)$, minimal endometriosis was present in $47(24.6 \%)$ women, mild in 39
(20.4\%), moderate in $29(15.2 \%)$ and severe in $76(39.8 \%)$. There was a significant improvement of VAS and QoL scores at 3 and 6 months postoperatively compared to preoperative scores for the whole group (Wilcoxon Signed Ranks Test; $p<0.01$ ). However, there was no statistically significant difference in the degree of improvement in the SF-36, VAS, SF-MPQ scores achieved at 3 and 6 months between the two groups (Mann-Whitney $\mathrm{U} ; p<$ $0.05)$.

Conclusions: Our results revealed no relationship between the rASRM score and improvement of symptoms after the laparoscopic surgery. Short and mid term response to laparoscopic surgery and improvements of the QoL scores at 3 and 6 months were statistically identical. Almost all patients reported improvement in their symptoms at 3 and 6 months irrespective of group allocation. There was significant improvement for all QoL scores. This was irrespective to good or a poor correlation between rASRM and QoL scores. Although well recognised from the fertility aspect, the widely accepted rASRM classification remains potentially deficient with regards to relationship with the pain symptoms of endometriosis.

\section{FC_46}

Can transvaginal sonography (TVS) predict infiltration depth in patients with deep infiltrating endometriosis (DIE) of the rectum?

G. Hudelist, K. Heinz Oberwinkler, F. Tuttlies, G. Rauter, O. Ritter, J. Keckstein

Center for Endometriosis, Villach General Hospital,

Villach, Austria

Introduction: Patients with DIE of the rectum often require extensive surgical treatment involving disc or segmental bowel resection. The aim of the present study was to evaluate the value of transvaginal ultrasound (TVS) for prediction of infiltration depth in cases of DIE affecting the rectum based on disruption of characteristic sonomorphological features of the rectal wall.

Materials and methods: Two-hundred patients with suspected DIE were prospectively assessed by TVS for the presence of rectal DIE before undergoing laparoscopic radical resection of endometriosis including segmental resection of the bowel in affected cases. Sensitivities, specificities, positive and negative predictive values (PPV, NPV) were then calculated for the presence of infiltration of the serosal (S), muscular (M) and submucosal/ mucosal (MUC) layers as demonstrated by TVS and confirmed by histopathological analysis.

Results: Bowel endometriosis was confirmed in 48 of 200 (24\%) cases with results of histopathological analysis of all bowel layers available in 44 of $48(91.6 \%)$ samples. Sensitivity and specificity, NPV and PPV of TVS in general was 91\%, 98\%, 93\% and 97\%. Data of TVS on S involvement were $93 \%, 99 \%, 98 \%$ and $98 \%$; data on $\mathrm{M}$ infiltration were $98 \%, 99 \%, 98 \%$ and $99 \%$; data on MUC were $62 \%, 96 \%, 53 \%$ and $97 \%$.

Conclusions: TVS is highly valuable tool in diagnosing rectal endometriosis preoperatively. Within this, serosal and muscular infiltration can be accurately predicted, whereas TVS is of little value for detection of mucosal involvement. 


\section{FC 47}

Disease management patterns and burden in women known to have fibroids: results from the CHASM (Cross-sectional survey of HRQoL and Symptoms of Myoma) study J. Gilabert Estellés $^{l}$, D. Subramanian ${ }^{2}$, E. Downes ${ }^{3}$, V. Sikirica $^{2}$, C. Maroulis ${ }^{2}$, S. C Bolge ${ }^{4}$

${ }^{I}$ University Hospital La Fe, Valencia, Spain, ${ }^{2}$ Ethicon, Somerville, NJ, USA, ${ }^{3}$ Chase Farm Hospital, Enfield, UK, ${ }^{4}$ Consumer Health Sciences, Princeton, NJ, USA

Objectives: To describe the management of women diagnosed with or experiencing symptoms suggestive of uterine fibroids (UF) and to quantify the burden experienced by this population.

Materials and methods: Data were collected from adult women residing in the US, Canada, and five European countries, diagnosed with UF (e.g., self-reported diagnosis or surgical/ medical treatment), or experiencing at least one of four menstrual symptoms on the UFS-Quality of Life (UFS-QoL) Symptom Severity Subscale or inter-menstrual bleeding. An internet-based questionnaire was used to quantify disease-specific and general quality of life (QoL; via SF-12v2), self-reported rates of healthcare resource use and treatment seeking patterns. The analyses presented here are limited to women who were diagnosed with UF. Results: Of 2,570 women who qualified for inclusion, a diagnosis of UF was self-reported by 1,053 women. Overall, age-weighted prevalence was $17.2 \%$ with the lowest individual prevalence in the UK $(12.2 \%)$ and the highest in Italy $(23.6 \%)$. Mean age varied from 35.9 years in Spain to 46.1 years in US, and the proportion of overweight/obese women varied from $32.4 \%$ in Italy to $75.8 \%$ in Canada. One fifth of all women overall (21.5\%) had experienced symptoms for at least 5 years prior to initiating treatment $(9.0 \%$ in Germany to $32.5 \%$ in Italy). The first provider consulted about symptoms was most often a general practitioner in Canada and the UK and a gynaecologist in France, Germany, Italy, Spain, and US. Across all countries, $55.9 \%$ (33.9\% in Spain to $69.4 \%$ in Canada) had a surgical intervention, $7.6 \%$ (4.8\% in Canada to $10.3 \%$ in France) used medication, and $36.5 \%$ (24.1\% in Germany to $57.4 \%$ in Spain) were untreated. In the past year, $57.4 \%(38.1 \%$ in UK to $75.9 \%$ in Germany) visited a provider and $18.2 \%$ (11.7\% in Spain to $35.2 \%$ in Germany) were hospitalized for uterine fibroids. Those who had undergone surgery had lower mean UFS-QoL symptom severity score $(34.9$ vs. $44.2 ; p<0.001)$ and higher mean HRQoL subscale score $(61.8$ vs. $53.8 ; p<0.001)$ than those on medication only.

Conclusion: While UF disease management patterns vary across countries, a substantial percentage of women have waited over 5 years before receiving treatment. Overall, women who have had surgery rather than medication have better QoL. Encouraging symptomatic women to seek treatment earlier is needed.

\section{FC_48}

\author{
Laparoscopic myomectomy after Doppler assessment \\ of fibroid blood supply \\ D. Tutunaru, B. Alexandru, I. Luiza Condulescu, \\ D. Florentina Lebit, A. Petca \\ Elias Emergency Hospital, Bucharest, Romania
}

Aim: To evaluate the usefulness of preoperative Doppler assessment of blood supply of large uterine leyomyomas.

Materials and methods: Prospective, randomised, group controlled study. Inclusion criteria: women with large uterine fibroids. Exclusion criteria: cervical severe dysplasia, genital malignancies, adenomiosis. Patients were randomly divided in two groups. Doppler assessment, with evaluation of fibroid blood supply and RI measurements of blood flow, was performed prior surgery using a VOLUSON 730 Expert BT05 system with a 5-9 MHz RIC5-9 curved linear array volumetric real time $3 \mathrm{D}$ endovaginal probe. We used a laparoscopic system provided by Karl Storz including $5 \mathrm{~mm}$ ceramic $\mathrm{RoBi}$ rotating bipolar forceps and scissors and monopolar scalpel for Autocon ${ }^{\circledR}$ II 400 Electrosurgical Unit. Laparoscopy was performed by one and the same surgeon. We compared duration of surgery, blood loss, intraoperator complications, postoperative recovery time.

Results: From 63 cases enrolled in the study, 33 were allocated to the study group and 30 to control group. Doppler assessment revealed 27 cases with individualised blood supply with measurable RI. (study group) and 26 cases respectively in the control group. In the study group bleeding was minimal due to targeted dissection of fibroid blood supply. Time of surgery was reduced due to minimal hemorrhagic events. Recovery period was shorter in the study group. There were no immediate or late complications in both groups.

Conclusion: Preoperative Doppler evaluation of fibroid blood supply proved to be a useful tool for adopting the best strategy during laparoscopic myomectomy.

\section{FC_49}

\section{Laparoscopic myomectomy: analysis of the risk factors of complications and conversion \\ F. Carmona, M. Angeles Martínez-Zamora, C. Ros, A. Osorio, C. Castelo-Branco, J. Antonio Vanrell, J. Balasch \\ Institut Clínic de Ginecologia, Obstetrícia $i$ Neonatologia. Hospital Clínic of Barcelona. University of Barcelona., Barcelona, Spain}

Objectives: To evaluate the surgical outcome and the safety of laparoscopic myomectomies and to find risk factors of conversion and complications.

Materials and methods: A prospective observational study including 208 patients who underwent a laparoscopic myomectomy from October 1994 to March 2008 in a tertiary referral centre. Myoma extraction was performed by using an electric morcellator.

Results: Mean number of myomas: $1.6 \pm 1.2$ (range 1-7). Mean size of the biggest myoma (mm): $72.3 \pm 23$ (range 43-150). Mean size of the sum of all myomas (mm): $86 \pm 33$ (range 58-240). The mean duration of surgery was $173 \pm 68.5 \mathrm{~min}$. There were 14 (6.7\%) conversions to laparotomy due to: size of the uterus: five; multiple myomas: five; haemorrhage: three; severe adhesions: one. No bladder or intestinal lesions or other iatrogenic organ damage were reported during the surgery. Immediate and short term complications were: febrile syndromes in 12 patients; four 
haemoperitoneums that required subsequent surgery (all myomas $>90 \mathrm{~mm}$ ); four hysterorrhaphy haematomas (all myomas $>$ $100 \mathrm{~mm}$ ) that did not require more surgery. 25 (12\%) patients had post-operative haemoglobin levels $\leq 8.0 \mathrm{~g} / \mathrm{dl}$ (all myomas> $90 \mathrm{~mm}) .17$ (8.1\%) patients required a blood transfusion. Inpatient stay post-operatively was on average $2.8 \pm 1.5$ days. The average follow up was $40.8 \pm 33$ months. Considering the $31 \%$ patients with a longer follow up of 12 months and with immediate gestational desire, $62.3 \%$ got pregnant. There were no uterine ruptures. The univariate and multivariate analysis showed as risk of complications (haemoperitoneum or haematoma requiring surgery, febrile syndrome, postsurgical $\mathrm{hb}<8 \mathrm{~g} / \mathrm{dL}$, requirement of blood transfusion) the diameter of the biggest myoma $(>70 \mathrm{~mm})$ and/or the mean size of the sum of all myomas $(\geq 5)$. The risk of conversion increased with the number of myomas $(\geq 5)$ and/or the diameter of the biggest myoma $(>70 \mathrm{~mm})$ and/or the mean size of the sum of all myomas $(\geq 130 \mathrm{~mm})$.

Conclusions: Laparoscopic myomectomy appears to be a safe technique with the advantages of short recovery time. The risk of conversion and complications increases with the number, the size of the biggest myoma and the size of the sum of all myomas.

\section{FC_50}

Bone anchor fixation In laparoscopic sacrocolpopexy $\underline{\text { R.M.F. van der Weiden }}^{l}$, M.I.J. Withagen ${ }^{2}$, E. Rociu ${ }^{1}$, G.H.H. Mannaerts ${ }^{1}$
${ }^{1}$ Sint Franciscus Gasthuis, Rotterdam, Netherlands, ${ }^{2} U M C$ St Radboud, Nijmegen, Netherlands

Introduction: Since 2004, we perform deep sacral mesh fixation (S-2/3) during laparoscopic sacrocolpopexy using bone anchor fixation. Experiences and initial results with the STORZ KS 00050-1/GYN prototype are presented.

Materials and methods: Forty-six patients underwent laparoscopic sacrocolpopexy with bone anchor fixation using a standard operative technique (Surgical Endoscopy 2005: 19: 594-597). 39 patients participated in a prospective study consisting of validated questionnaires, urodynamics, POP-Q staging and dynamic MRI before and at least 3 months after surgery. Median age at the time of surgery was 67.5 years (range $45-86$ years).

Results: Up to March 2008, 32 patients completed the study. Median operative time: $120 \mathrm{~min}$ (range 60-240 $\mathrm{min}$ ); decreasing with the STORZ prototype to $90 \mathrm{~min}$. Median blood loss: $50 \mathrm{ml}$ (range $50-100 \mathrm{ml}$ ). A serosal lesion of the ileum necessitated conversion in one patient. Another patient had temporary neurological complaints due to placement of the bone anchor close to a sacral foramen. Both patients made an uneventful recovery. Vaginal vault prolapse recurred in four patients. Following surgery, cystometric capacity increased and residual volume decreased. De-novo stress incontinence and/or urgency were not observed.

Discussion: Bone anchor fixation facilitates deep sacral mesh placement in laparoscopic sacrocolpopexy. Results are comparable with traditional sacrocolpopexy. The use of the STORZ prototype KS $00050-1 /$ GYN significantly reduces operative time.

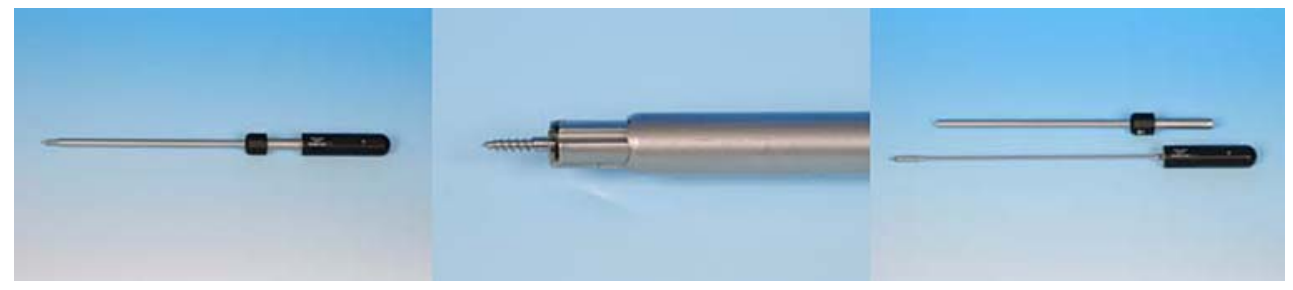

\section{FC 51}

NOTES transvaginal access: exploration of pelvis and future applications in gynaecology field C. Zacharopoulou ${ }^{I}$, J. Nassif ${ }^{1}$, S. Perretta ${ }^{2}$, B. Dallemagne $^{2}$, J. Marescaux ${ }^{1}$, A. Wattiez ${ }^{1}$ ${ }^{I}$ IRCAD/EITS, strasbourg, France, ${ }^{2}$ UNIVERSITY LOUIS PASTEUR, strasbourg, France

Objective: To report initial results of transvaginal access for n.o.t. e.s (natural orifices transluminal endoscopic surgery) cholecystectomy in human beings, evaluation of retroflexed view of the pelvis and future procedures in gynecology.

Materials and methods: From April 2007 to April 2008 a total of six n.o.t.e.s cholecystectomies, using the trasvaginal access, were performed in six patients with symptomatic cholelithiasis in our hospital. Exclusion gynaecologic criteria included rectovaginal endometriosis, fixed retroverted uterus and mass in the pouch of Douglas. With the patient in steep Trendelemburg position, under laparoscopic control, the transvaginal access was achieved via a $2 \mathrm{~cm}$ posterior colpotomy performed $1 \mathrm{~cm}$ below the cervix. The peritoneal cavity and organs were explored by a double channel scope in retroflexed position. After cholecystectomy procedure, the colpotomy was closed with separate stitches of Vycril 2/0.

Results: All transvaginal accesses were successfully performed with no intraoperative complications related to the access and closure technique. A excellent view of the pelvic organs as ovaries, tubes, utero-vescical pouch and pouch of Douglas was obtained. Postoperative course was uneventful, with no bleeding, infection, discharge and dyspareunia reported at a mean follow up of 7 months.

Conclusion: Since n.o.t.e.s became a reality in clinical practice, gynecologists became fundamental in the team. The positive 
patients outcome without any gynecologic complication and the development of new flexible endoscopes opens the way to transvaginal n.o.t.e.s procedure in gynecology that are currently performed by laparoscopy. The experience gained in our series of six patients allowed us to explore other possible gynecological targets. Study of suspect ovarian mass, tubal ligation, treatment of hydrosalpinx, para-tubal cysts, ovarian cysts, ectopic pregnancy could represent the target of n.o.t.e.s procedures in the future.

\section{FC_52}

Robotic total hysterectomy and robotic prolapse surgery with the Da Vinci ${ }^{\circledR}$ surgical system:

a prospective evaluation of the first 30 cases

D. Sarlos, L. Kots, N. Stevanovic, G. Schär

Department of Gynecology Kantonsspital Aarau, Aarau, Switzerland

Introduction: While robotic surgery has manifested itself in urology and cardiac surgery this technology still needs to be evaluated for gynecologic surgery. There are few studies on this topic and the most are retrospective with a small number of cases. The aim of this prospective pilot study was to evaluate perioperative outcome of Robotic procedures in an institution experienced in advanced endoscopic surgery.

Materials and methods: We started to perform DaVinci ${ }^{\circledR}$-Robotassisted surgery since June 2007. Perioperative data of the first 30 consecutive cases were prospectively evaluated. 25 patients had total hysterectomy for benign reasons and five patients had sacrocolpopexy because of symptomatic post hysterectomy prolapse.

Results: Mean duration of robot installation was 23 min (range 5$60 \mathrm{~min}$ ), mean duration of total surgery was 173 min (110$200 \mathrm{~min}$ ), mean duration of vaginal cuff closure in cases of hysterectomy was $18 \mathrm{~min}$. Patients who received total hysterectomy were hospitalized for 3 days (2-5 days) respectively 4 days when sacrocolpopexy was performed. There were no conversions to conventional laparoscopy and no conversions to laparotomy. There were no relevant intra- and postoperative complications.

Conclusions: Da Vinci- ${ }^{\circledR}$-Robot assisted procedures seem to be feasible for gynaecologic surgery. The perioperative outcome seems to be equal to the outcome after conventional laparoscopic surgery. For the experienced endoscopic surgeon the method does not seem to have relevant advantages. We assume that Robotic surgery has an enormous potential for the future but needs further evolution.

\section{FC 53}

\section{More than meets the eye}

T. Koninckx ${ }^{l}$, M. Moll ${ }^{2}$, L. Van Gool ${ }^{2}$, P. Koninckx ${ }^{2}$

${ }^{I}$ eSaturnus, Leuven, Belgium, ${ }^{2}$ KULeuven, Leuven, Belgium

Endoscopy has a reputable and longstanding history. However, after its breakthrough in the 1980 s with the introduction of compact solid state cameras, the technology did only gradually evolve. An endoscopic imaging system, still rather is a prolongation of the eye, and not a true cognitive sensor.

With this work we demonstrate two new computing algorithms on real endoscopic data showing that endoscopic video data carries more information than what the human observer can see. Firstly an application which highlights the vascularization within endoscopic video footage is presented. It is to be considered a first step of intelligent video processing, facilitating the medical diagnosis by the surgeon. In contrary to related systems (e.g. NBI) this new approach is based on computer based image interpretation only. No special cameras or illumination are involved.

Secondly, we demonstrate how the video footage itself can be used to localize the $3 \mathrm{D}$ orientation of the camera relative to the intra abdominal scene. This so-called ego-motion estimation algorithm is used to facilitate the application of $30^{\circ}$ laparoscopes. It is well known that the oblique scopes can be effectively used to improve the viewing range by rotating the scope. In this case, the camera has to be kept fixed so that the captured video does not rotate as well. As both the surgeon and the assistant use both their hands during surgery, it means that one of them has to interrupt his task in order to rotate the scope against the camera. Exactly this unnecessary and cumbersome handling is removed using image processing. We propose a method to detect and undo rotations of the image automatically and in real-time, thereby allowing to perform the same action with only one hand.

This work is based on practical research, which has been clinically validated. It clearly demonstrates that by combining medical expert knowledge and advanced engineering it becomes feasible to build intelligent endoscopic video systems. It might be the start of a new series of value adding assisting systems during surgery...

\section{FC 54}

\section{Comparison of two electric morcellators} for laparoscopic gynaecological surgery M. Angeles Martínez-Zamora, Al. Izquierdo, C. Castelo-Branco, F. Carmona

Institut Clínic de Ginecologia, Obstetrícia i Neonatologia. Hospital Clínic of Barcelona. University of Barcelona., Barcelona, Spain

Objectives: To compare the efficiency and safety of a newly developed reusable morcellator with a disposable morcellator for minimally invasive supracervical hysterectomy and miomectomy. Materials and methods: We performed a prospective, randomized parallel-group study including 30 patients. 15 patients were treated with the Gynecare Morcelex ${ }^{\circledR}$ laparoscopic disposable morcellator (group 1: 11 myomectomies and four supracervical hysterectomies) and 15 with the Olympus Variocarve ${ }^{\mathbb{R}}$ morcellator (group 2: 12 miomectomies and three supracervical hysterectomies).

Results: There were no statistical differences between both groups in the total tissue weight (395 versus $410 \mathrm{~g}$ ). The rate of morcelation was statistically higher in group $2(30.5 \mathrm{~g} / \mathrm{m}$ versus $41 \mathrm{~g} / \mathrm{m} ; p<0.05)$. The morcellating time was significantly shorter in group 2 (13 versus $10 \mathrm{~min} ; p<0.05)$. Significantly fewer and longer pieces of tissue $(p<0.05)$ were removed with the Olympus Variocarve $^{\circledR}$ morcellator (Length of pieces in cm: 4.9 vs 7.8; percentage of pieces longer than $5 \mathrm{cms}$ : 43 vs 72). No bladder or intestinal lesions or other iatrogenic organ damage were seen with either morcellator. None of the patients in either group had postoperative complications. All interventions were completed as 
planned in both groups, and none of the procedures had to be converted to an open operation. Adequate tissue for hystologic analysis was obtained from all patients.

Conclusions: The Olympus Variocarve ${ }^{\circledR}$ morcellator is a safe and effective instrument for laparoscopic miomectomies and supracervical hysterectomies, offering a much shorter operation time for the removal of morcellated tissue than a conventional device and a low risk of injury to surrounding organs and tissue.

\section{FC_55}

\section{NOTES in Gynecology}

A. Hackethal $^{l}$, S. Langde ${ }^{1}$, G. Tcharchian ${ }^{2}$, I. Hrgovic ${ }^{1}$, $\overline{\text { K. Muenstedt }}^{1}$, H.-R. Tinneberg ${ }^{1}$

${ }^{I}$ University Giessen, Giessen, Germany, ${ }^{2}$ Pius Hospital Oldenburg, Oldenburg, Germany

Objective: Natural Orifice Transluminal Endoscopic Surgery might be associated with potential benefits to the patients and professional health systems. Entering the abdominal cavity with a transvaginal, transgastric, transrectal, transvesical or with a hybrid approach are less atraumatic than the conventional and laparoscopic approaches. The absence of pneumoperitoneum might even add to a reduction of adhesion formation. The transvaginal surgery is standard of care for gynecologists. Beyond the usual transvaginal approach, we introduce a less traumatic and more flexible transvaginal NOTES.

Results: During the last month, a bilateral salpingooophorectomy and an appendectomy have been performed interdisciplinary via NOTES at the Gynecological department of the University clinic of Giessen. Surgery seems feasible, the postoperative course proved to be excellent.

Conclusion: Laparoscopic surgery revolutionized modern surgery. What was believed to be impossible at first, proofed to be as effective and rational during the next 20 years. Now a new verge is accomplished and the NOTES technique might lead to revolutionize surgical behaviour again. Before, NOTES for gynecologists needs to persuade the classical vaginal and the advanced laparoscopic surgeons.

\section{FC_56}

\section{Investigation into the usability of medical devices} in the application range $O R$

U. Matern ${ }^{1}, \underline{\text { K. Montag }}{ }^{1}$, T. Rölleke ${ }^{2}$, B. Schauf ${ }^{1}$, P.-S. Mauz ${ }^{1}$, D. Wallwiener ${ }^{1}$

${ }^{1}$ Experimental-OP und Ergonomie, Universitätsklinikum Tübingen, Tübingen, Germany, ${ }^{2}$ BfArM, Federal Institute for Drugs and Medical Devices, Bonn, Germany

This study is concerned with the usability of medical devices, currently used in OR today and the question how far the medical devices follow the demanded usability criteria for suitability of task, self description ability, controllability, expectation conformity, error robustness etc.

In excess of 900 cases will be investigated in the study, all of which were referred to the BfArM since the year 2000. Initial evaluation of the first 306 cases shows that the user was the reason for the incident $32 \%$ of the time and in $23 \%$ of the messages the reason was false communication between user and medical device. Concerning the user errors a wrong operational application by the user outweigh far with $80 \%$ followed by incorrect cleaning and sterilization with $10 \%$.

False practice dominates the application error category, however in these cases the medical device is often set-up in such a way that promotes a false user procedure. The medical device itself gives the user no chance to realise that it is not suitable for the application and mistakes are made.

One hundred forty of the patients in the first 306 cases were so badly injured that they required additional surgery or supervision in intensive-care. In $70 \%$ of the cases the OR time was clearly prolonged and $12 \%$ of the patients even had to be re-admitted for further treatment.

There are however rarely consequences for the medical devices and they continue to be used unchanged. In fact $29 \%$ of future products and only $17 \%$ of the products used in the field have been modified. Often the only modification that was made, was simply a supplement of the operating instructions and it makes one wonder, whether the modification reaches the user at all?

The cases investigated demonstrate the importance of user training - it is vital that users are more intensively trained (and training must be refreshed/kept up-to-date). The use of the instrument or device must be clear and the user must be in no doubt as to how the instrument is to be used. Standardization of the user interface can prevent confusion resulting from a change of device, as users are familiar with similar devices that they have seen before.

\section{FC_57}

The Experimental-OR in Tuebingen-scientific background and introduction of the institution

B. Schauf, P.-S. Mauz, U. Matern, D. Wallwiener

Experimental-OR and Ergonomics, University Hospital Tuebingen, Tuebingen, Germany

The operating room is one of the most expensive and most complex units within a hospital. To safely and efficiently operate an OR all components (building and equipment technology) should be harmonized with each other. Own experience being a surgeon, showed that this is not the case. To analyze this hypothesis and to accordingly draw the resultant conclusions various investigations were performed using custom-built tools. Surgeons and OR staff were surveyed using standardized questionnaires: $70 \%$ of surgeons and $49 \%$ of OR staff are not able to manipulate the medical technology correctly. They argue that this is leading to a high potential of hazards of all persons within the OR.

To ensure this, the critical incident reports to the BfArM (Federal German Institute for Drugs and Medical Technology) between the year 2000 and 2006 were statistically analyzed. We found 1,330 reports. The reason for about $50 \%$ of the reported cases was a "misunderstanding" between human and machine-a design, teaching or training problem. 
The preliminary result of their analysis is that over $90 \%$ of the cases were combined with a dangerous situation for the patient; about $12 \%$ of the patients needed a second procedure. In $70 \%$ of the cases the OR time was clearly prolonged.

We can conclude that ergonomic problems within the OR are not only a safety issue but although an economic problem.

Therefore, at the University Hospital Tuebingen the worldwide unique laboratory - the Experimental-OR - is implemented. The new department ,Experimental-OR and Ergonomics "plans, builds and operates this laboratory and coordinates the various projects. A $400 \mathrm{~m}^{2}$ OR unit consisting of two ORs and the according auxiliary rooms, is set up in a $1,000 \mathrm{~m}^{2}$ factory floor. The Experimental-OR is a lab to improve and to integrate medical and building technology to one ergonomic and economic efficient system. No patient will be brought into danger by the continuous research and development of new technology in a very early state of its development. Planer, architects and industry are involved in the Experimental OR. The recent list of project partner can be found on www.experimental-op. de. The Experimental-OR is an international open platform.

Within the presentation an overview will be given about the current situation and a future prospect about the further development.

\section{FC_58}

\section{The SECURE study: ultrasound evaluation}

\section{of the caesarean scar}

M. Bij de Vaate, H. van der Slikke, J. Huirne, H. Brölmann

VU University Medical Center, Amsterdam, Netherlands

Objective: While the caesarean section (CS) rate is increasing in most Western countries, the long-term effects of this procedure are poorly studied. In almost $60 \%$ of women with a caesarean scar, ultrasonography shows a 'niche'. A niche is defined as a triangular, anechoic area at the presumed site of incision. It is suggested that a niche may be responsible for abnormal uterine bleeding. To evaluate the consequences of a niche, the SECURE-study (Scar Evaluation after Caesarean by Ultrasound REgistration) was started in October 2007. The primary objective of this study is to develop a niche classification and evaluate the relation with abnormal uterine bleeding.

Materials and methods: In an observational prospective cohort study, women are examined by gel instillation sonohysterography (GIS) 6 to 12 months after CS to detect a niche. The women are not informed about the presence of a niche by the investigator. A questionnaire is completed and women keep record of their bleeding pattern for a period of two cycles. The questionnaire will be repeated every year for the next 5 years.

Results: An interim analysis was performed after inclusion of 68 women, which demonstrated the presence of a niche in 38 women (55.1\%). Examples of different types of niches (2D/3D) will be shown. In the group of women with niche the prevalence of intermenstrual bleeding was $42 \%$, and in the group of women without niche $23 \%$.

Conclusions: These preliminary results show that a niche is seen in $55.1 \%$ of women with a caesarean scar, and that intermenstrual bleeding is more frequently present in women with niche.
FC_59

\section{Development and testing of ergonomic hand-instrument interfaces for surgery \\ U. Matern, B. Schauf, P.-S. Mauz, D. Wallwiener \\ Experimental-OR and Ergonomics, University Hospital of Tuebingen, Tuebingen, Germany}

Introduction: Ergonomic deficiencies of surgical instruments leading to nerve irritations, pressure areas, muscle cramps and shoulder pain, have been described in detail in publications and are well known among surgeons. The shape of the instruments' handles as well as their effect on the posture of the surgeon, especially of his arm and hand, can be identified as causes of these deficiencies. The goal of research is to evaluate the technical reasons for these deficiencies and to develop new surgical instruments considering basic rules of ergonomics.

Materials and methods: Based on the literature on ergonomics, basic knowledge, personal experience and scientific testing criteria for functional, comfortable and ergonomically satisfactory surgical instruments have been defined. For scientific studies within the OR and ergonomic lab EMG, ultrasound tracking, video based observation and questionnaires were used. Specific test modules as well as VR (virtual reality) trainers for surgery were established.

Results: Over 20 criteria for the evaluation and designing new handles of surgical instruments were established. These criteria effect the ergonomics of the interface between surgeon and instrument like handling, sensitivity, force feedback and posture. In cooperation with industrial designers and partially with manufacturers of surgical instruments several new handles have been developed and tested. These handles fulfill some of the criteria and diminish ergonomic deficiencies.

Discussion: Scientific measurements and comparison within the $\mathrm{OR}$ and the lab are basic tools to recognize problems with handling of currently available handles or prototypes and to describe new criteria for ergonomic engineering. It is possible to design and shape models of new handles that fit all criteria. The technical realization is difficult, because forces necessary often can not be archived with mechanical constructions currently used in industry. Therefore it is important to find new technical solutions to create better ergonomic man machine interfaces.

\section{FC_60}

Usability of radio-frequency devices in surgery

U. Matern, D. Büchel, B. Schauf, P.-S. Mauz,

D. Wallwiener

Experimental-OR and Ergonomics, University Hospital Tuebingen, Tuebingen, Germany

This paper describes the usability evaluation conducted at the Experimental-OR of the University Hospital Tuebingen. Subjects of the study were three radio-frequency (RF) devices for surgery. The aim of this study was to detect the differences in the usability among devices which have the same mode of operation and to announce the usability problems occurring with their use. These 
tests also provide key data for the improvement in further development of RF devices.

\section{FC_61}

Comparison of diagnostic use of a newly developed device offering a combination of endometrial sampling and subsequent saline infused sonography (BiopSIS $\left.{ }^{\circledR}\right)$ with the conventional serial use of endometrial biopsy with subsequent saline infused sonography by two different devices

D. Schoot, V. Dietz

Catharina hospital, Eindhoven, Netherlands

Introduction: Randomized study comparing the diagnostic use of a newly developed device offering a combination of endometrial sampling and subsequent Saline infused sonography (BiopSIS ${ }^{\circledR}$ Medical Dynamics) with the conventional serial use of endometrial biopsy with subsequent saline infused sonography (SIS) by two different devices (Goldstein catheter (GC) by Cook $^{\circledR}$ and Pipelle de Cornier $\left.^{\circledR}\right)$.The newly developed "ready to use" diagnostic device was expected to have similar diagnostic value, but more efficient and more patient friendly.

Materials and methods: Fifty-one subjects with first time postmenopausal bleeding and increased endometrial thickness $(>4 \mathrm{~mm}$ ) were randomized to one of two groups. Two groups of women completed endometrial sampling followed by SIS using both diagnostic modalities [BiopSIS ${ }^{\circledR}(n=27)$ or endometrial biopsy followed by SIS $(n=24)]$. At the end of the procedures, a pain questionnaire was completed. Histology was reviewed in a blinded fashion. Sonograhic results were correlated to following hysteroscopy. Procedure time was compared between groups. Use of devices was evaluated by experienced gynaecologists.

Results: Devices could not be introduced in three patients (2 Pipelle 1 BiopSIS $\left.{ }^{\circledR}\right)$. Duration of the preparation and procedure time was significantly shorter in the BiopSIS ${ }^{\circledR}$ group (5.4 vs. $8.7 \mathrm{~min}$ ). In each group, two endometrial samplings demonstrated not enough tissue for diagnosis. The instruments yielded biopsies of similar quantity and quality as judged by pathologists. Pain scores were similar using both diagnostic algorithms (6.7 vs. 6.0: Visual Analogue Score). Hysteroscopic evaluation after sonohysterography demonstrated no unexpected abnormalities in both groups. The choice to perform a SIS directly following endometrial sampling was appreciated by a majority of gynaecologists (66\%).

Discussion: In more then $35 \%$ of abnormal postmenopausal bleeding, the uterine cavity shows abnormalities which lead to diagnostic and therapeutic hysteroscopy. Recent studies show that combining Saline infused sonography with sampling can be of more use in preventing repeated blood loss caused by polyps.

Conclusion: Combined endometrial sampling/SIS device can give quicker, more patient friendly and reliable results.

\section{FC_62}

\section{Force of tissue graspers on laparoscopic surgery}

D. Ott

Mercer University, Macon, Georgia, USA
Objective: To assess the grasping strength of laparoscopic instruments.

Materials and methods: Measurement and analysis of laparoscopic grasping instruments grasping force (crush strength) and tissue deformation was done by using a strain gauge with force feedback capability as a thin film load cell or piezo-resistive sensors incorporated into laparoscopic grasping devices to measure load pressure during use. Measurement of laparoscopic grasping force and tissue deformation characteristics were also assessed.

Results: Grasp strength equalled crush strength for all instruments on all tissues tested.

Conclusions: Grasping instruments used during laparoscopy create excessive load pressures on tissues within their grasp manipulating tissue in one crush strength fits all circumstance manner. Laparoscopic grasping instruments force profile should be modified to allow tissue stabilization without ischemic effects and with minimal tissue damage. The force required to stabilize, pull, lift and move tissue is significantly less than that generated by currently available laparoscopic grasping instruments.

\section{FC_63}

What caused failure in outpatient hysteroscopy S. S M Wong $^{I}$, S. E Jones ${ }^{2}$

${ }^{1}$ Calderdale Royal Hospital, Halifax, UK, ${ }^{2}$ Bradford Royal Infirmary, Bradford, UK

Introduction: The introduction of outpatient hysteroscopy clinics has dramatically reduced the need of hysteroscopy performed as an in-patient and day-case bases. However, there are still cases where outpatient hysteroscopy is not possible. We aim to study the causes and associated factors in these cases to assist patient selection in the future.

Materials and methods: Information on women who had hysteroscopy (Versascope) performed in an outpatient hysteroscopy clinic within a teaching hospital between 2003 and 2007 were collected retrospectively. The data were stored and analysed using the SPSS package.

Results: Within the 54 months duration, 3,694 cases of hysteroscopy were performed in the clinic and 333 cases $(9 \%)$ of failed procedure were reported. The causes of failure included: 219 cases $(66 \%)$ of cervical stenosis, 65 cases $(20 \%)$ of pain or discomfort, 36 cases $(11 \%)$ of poor view, 28 cases $(8 \%)$ of inability to visualise cervix, 19 cases $(6 \%)$ of inability to pass speculum, 15 cases $(5 \%)$ of acutely anteverted or retroverted uterus and eight cases $(2 \%)$ of vaso-vagal attack. Failed procedure were more likely if the women were older $(p<0.01)$, nulliparous $(p<0.01)$, fast tract referral $(p<0.01)$, with postmenopausal bleeding $(p<$ $0.01)$ and had previous endometrial ablation $(p<0.01)$. Failed procedure were less likely if the women were from direct access referral $(p<0.01)$, with intermenstrual bleeding $(p=0.02)$, not required local anaesthetic usage $(p<0.01)$ and not required cervical dilatation $(p<0.01)$.

Conclusion: The number of failed procedure was low. Some of the failed procedure can possibly be prevented by careful patient selection, more usage of local anaesthesia, performing vagino- 
scopy instead of using speculum and availability of a flexible hysteroscope in clinic. However, the associated factors located were mainly reflecting the underlying pathology and technical difficulty, which would not be avoidable but would be important factor to acknowledge especially for training purpose.

\section{FC_64}

Patients' preferences for salpingostomy relative to salpingectomy in tubal ectopic pregnancy: a discrete choice experiment

$\underline{\text { N.M. van Mello }}^{1}$, F. Mol${ }^{1}$, E. Edelman ${ }^{1}$, B.C. Opmeer ${ }^{2}$, W.M. Ankum ${ }^{1}$, F. van der Veen ${ }^{3}$, B.W. Mol ${ }^{1}$, P.J. Hajenius ${ }^{1}$

${ }^{I}$ Department of Obstetrics and Gynaecology, Academic Medical Center, University of Amsterdam, Amsterdam, Netherlands, ${ }^{2}$ Department of Clinical Epidemiology and Biostatistics, Academic Medical Center, University of Amsterdam, Amsterdam, Netherlands, ${ }^{3}$ Center for Reproductive Medicine, Academic Medical Center, University of Amsterdam, Amsterdam, Netherlands

Introduction: Whether tubal ectopic pregnancy (EP) should be treated by laparoscopic salpingostomy or by salpingectomy from the perspective of future natural pregnancy chances, is subject to ongoing debate. It is unknown which treatment women with a desire for future pregnancy prefer, thereby trading off the presumably better fertility outcome after salpingostomy against the potential disadvantages of this treatment, i.e. persistent trophoblast (PT) and an increased risk for repeat EP in the same tube as compared to salpingectomy. We investigated this question by means of a discrete choice experiment (DCE) for patients' preference.

Materials and methods: We performed an index-control study. The index patients were women surgically treated for tubal EP as part of an ongoing randomised controlled trial (ESEP study, ISRCTN37002267). The controls were non pregnant women at risk of EP visiting an infertility clinic. All women were offered a webbased DCE of 16 choice sets. Each choice set consisted of two profiles representing hypothetical scenarios of salpingostomy. An 'opting out' option, representing the values in case of salpingectomy, was the same for every choice set. A conditional logit regression model was used to analyse relative importance of the attributes.

Results: We included 49 index patients who were treated by either laparoscopic salpingostomy or salpingectomy for tubal EP. At present, five controls are included, but the inclusion of controls is still ongoing. For the index patients, spontaneous IUP rate and risk of repeat EP in the same tube were important for women's choices, coefficients $0.054(0.045$ to 0.064$)$ and $-0.136(-0.156$ to -0.115$)$, respectively. The coefficient for PT was not contributing to responders' preference. When calculating the trade-offs, women were willing to trade the risk of a repeat EP only if the spontaneous IUP rate increased. In case of 5\%, 10\%, 15\% risk of repeat EP, womens' preference shifted from salpingectomy to salpingostomy when the chance of spontaneous IUP was more than $33 \%, 45 \%$ and $58 \%$, respectively.

Conclusion: Women who have been surgically treated for EP were willing to accept the burden of a repeat EP in the same tube, but only if the increased risk of a repeat EP was compensated by an increase in IUP rate of 2.5 times this EP risk.
FC_65

Laparoscopic sacrocolpopexy: anatomical results, quality of life and perioperative outcome - a prospective study with 101 cases

D. Sarlos, S. Brandner, L. Kots, N. Gygax, G. Schaer

Kantonsspital Aarau, Department of Obstetrics\& Gynecology, Aarau, Switzerland

Objectives: Vaginal vault prolapse is a common problem in Gynaecology. The multitude of surgical options and lack of randomized trials make it difficult to decide between vaginal, abdominal and laparoscopic approach and whether to use a mesh. Recent advances in laparoscopy offer an interesting opportunity. In our prospective clinical trial we evaluated laparoscopic sacrocolpopexy considering perioperative data, objective anatomical results and postoperative quality of life.

Materials and methods: In our study 135 patients were operated by laparoscopic sacrocolpopexy between October 2003 and July 2007. Preoperatively each patient received an urodynamic screening including perineal ultrasound and a clinical follow-up 3, 6, 12 and 24 months after surgery. For each patient the vaginal prolapse was staged before and after surgery according to the POP-Q system and quality of life was assessed using the Kings Health questionnaire.

Results: One hundred one patients completed the study with an average follow up of 12 months: 46 had sacrocolpopexy only, 55 had simultaneous supracervical hysterectomy. Mean duration of surgery was $141 \mathrm{~min}$ respectively $154 \mathrm{~min}$ for the procedure including supracervical hysterectomy. Mean duration of hospitalisation was 4.6 days. According to the ICS classification subjective cure rate was $93 \%$ and the objective cure rate $98 \%$. We had a recurrence rate of $6 \%$ in the anterior compartment; only two were symptomatic. No apical and only two asymptomatic recurrences of the posterior compartment were found. De novo dysparenuria and mesh erosion occurred in less than $1 \%$. Main complications included four bladder and three rectal lesions.

Conclusions: Laparoscopic sacrocolpopexy is an excellent option for treatment of symptomatic apical prolapse. The rate of mesh erosion and de novo dysparenuria is negligible making it the method of choice for young and sexually active patients. As severe intraoperative complications may occur it requires sufficient experience in laparoscopic surgery.

FC_66

Indication of laparoscopic re-operation after sacrocolpopexy C.Gastaldi, E.Baulon, V.Thoma, J-J.Baldauf, A.Wattiez

Centre hospitalier universitaire de Hautepierre, Strasbourg, France

Introduction: Several studies have shown that sacrocolpopexy is associated with a low complication rate(5-10\%) including vaginal mesh exposure, de novo urinary stress incontinence or prolapse recurrence. Most of these complications can be treated vaginally. However, in some cases abdominal surgery may be unavoidable. The aim of this study was to assess the indications for re-operation by laparoscopy following a sacrocolpopexy performed either by laparotomy or laparoscopy. 
Materials and methods:We conducted a retrospective monocentric study, at the Centre hospitalier universitaire de Strasbourg, France. We identified seven patients with laparoscopic surgery for any complication after an abdominal sacrocolpopexy, from January, 1st 2004 to March, 31st 2008. Previous pelvic surgery, sacrocolpopexy procedure (number of meshes), clinical symptoms, time between sacrocolpopexy and re-operation, indication of re-operation and per-operative procedure were studied.

Results: Quite all patients (six of seven) had undergone previous pelvic surgery. Indications of the re-operation were: mesh exposure(four of seven) with vaginal fistula through anterior and posterior meshes (three of seven), prolapse recurrence(two of seven), and chronic cystalgy (one of seven). Mean time between sacrocolpofixation and re-operation was 22 months (3 to 58 months). Removal of one part or of the totality of the mesh was necessary in six cases. Despite this new procedure, three patients have shown post-operative complications.

Conclusion: Sacrocolpopexy for treatment of the prolapse in the young women appears to be an efficient technique with low rate of complications, but, when it occurs, a total removal of the mesh by laparoscopic surgery seems to be necessary. All the patients of the study underwent a sacrocolpopexy with two meshes. In three cases we reported a fistula at the union of two meshes. Futures studies should evaluate to perform sacrocolpopexy with one mesh fixed to the promontory.

\section{FC_68}

Accident analysis: the contributing factors of a ureter injury during deep endometriosis surgery

$\underline{\text { R. Schonman }}^{1}$, C. De Cicco ${ }^{1}$, R. Corona ${ }^{1}$, D. Soriano ${ }^{2}$, P. R Koninckx

${ }^{I} K U$ Leuven, Leuven, Belgium, ${ }^{2}$ Sheba Medical Center, Tel Hashomer, Israel

Introduction: Surgical mistakes occur, but the analysis of the contributing factors has rarely been done systematically.

Materials and methods: The analysis of a video recording of the entire laparoscopic surgery was performed to identify changes in surgical behaviour and factors contributing to a ureter injury. The video was reviewed independently by six expert gynaecologists. A 26 year old woman with severe pain was operated for a big $40 \mathrm{~mm}$ rectovaginal endometriotic nodule involving the left ureter and the rectum. On day 2 after surgery a repeat laparoscopy was performed to rule out a late bowel perforation. On day 5 after surgery a urine leak developed, a left ureter transection was diagnosed and a laparoscopical ureteral anastomosis performed. Further outcome was uneventful. Results: Surgery was performed as the third intervention after already two very demanding interventions for deep endometriosis. Analysis of the video-recording demonstrated that during the intervention the duration of uncontrolled bleeding $(P<$ $0.0001)$, defined as bleeding that should have been coagulated immediately, increased during the laparoscopy. Also the duration of laser activation $(P=0.013)$ increased. The simultaneous activation of laser cutting and bipolar coagulation occurred only at the end of surgery ( $n=7$ episodes). Unfortunately fatigue could not directly be measured, although it is obvious after $11 \mathrm{~h}$ of surgery.
Conclusion: Unconscious acceleration of surgery was evidenced by longer periods of uncontrolled bleeding, of laser activation and of performing simultaneously cutting and coagulation. It is suggested that this occurs as a consequence of fatigue and that this is a factor contributing to the error of judgment.

\section{FC 69}

Evaluation of manual laparoscopic skills using the Giessener Bench Station-training

Z. Doering, J. Ionesi, G. Tcharchian, C. Münstedt, H.-R. Tinneberg, A. Hacketal

University of Giessen, Giessen, Germany

Objective: Assessing surgical skills is not feasible. Methods for skills assessment consist of log books, surgical or video observation with or without criteria in different valid and reliable proportions. The criteria based video assessment reaches almost valid and reliable proportions. To establish a skill assessment tool for course participants of the Giessen School of Endoscopic Surgery, we set up a bench station training and evaluated manual skills with defined criteria.

Design: Die Giessen Bench Station-Training consists of four training tasks. The first task consist of placing peas on golf teas, the second task involves a needle manoeuvering through different aligned eyelets, the third task of cutting a triangle figure out of an examination glove and the fourth task of performing a single knot suture with two safety slings. Thirty students, 30 advanced surgeons (laparoscopy course participants) and 30 experts $(>300$ operative laparoscopies) performed the four tasks under standardized conditions. The performance was videotaped and analysed by trainers and experts ( $>300$ operative laparoscopies) with the help of 61 defined criteria.

Results: Results show a correlation of laparoscopic surgical experience and predefined criteria. As an example, criteria and results for the pea placement task: How many peas have been lost unintentionally from the instruments during movement? (Novice: 5.7, advanced surgeons: 3.27 and expert: 0.45). Time for first task completion: (Novices: $430.5 \mathrm{~s}$, advanced surgeons: $248.4 \mathrm{~s}$ and experts: $116.8 \mathrm{~s}$ ). With the help of the defined criteria, we are able to differentiate between novices, advanced surgeons and experts.

Conclusions: With the defined criteria we were able to assess the participants performance and differentiate levels of expertise. After proof of validity and reliability of the Giessen Bench Station-Training, we are able to use the test as assessment tool during laparoscopy workshops.

Keywords: Objective skills assessment, laparoscopic surgery, bench model, training

\section{FC 70}

\author{
Laparoscopic complication evaluation throughout an 18 years \\ follow up \\ L. Gonzalez Gea, J. Jose Delgado, J. Manuel Menéndez, \\ P. Garbayo, R. Cajal, A. Zapico \\ Príncipe de Asturias Hospital. School of Medicine. Alcalá \\ University, Alcalá de Henares. Madrid, Spain
}


Introduction: Laparoscopic procedures starting up programs have usually been related to an increasing complication rate when compared to traditional approach. Therefore, some changes in the department global learning curve should be related to the widespread of the procedure to all the team members.

Materials and methods: Obstetrics and gynaecology department of a General District Hospital. Retrospective review from 1990 to 2007 of 1,628 laparoscopies. Evaluation of the complication rate according to the starting up, teaching and formal standardization of this technique.
Results: Ninety eight procedures over 1,628 were due to malignant diseases. The remaining 1,530 laparoscopies were mainly due to adnexal benign pathology, myomas, ectopic pregnancy, etc. Global complication rate was $7.34 \%$. At the beginning of the experience, few complications were seen. However, widespread of the technique became into an increasing number of complications. The same trade was seen after 1999 when oncological procedures came into a new indication.

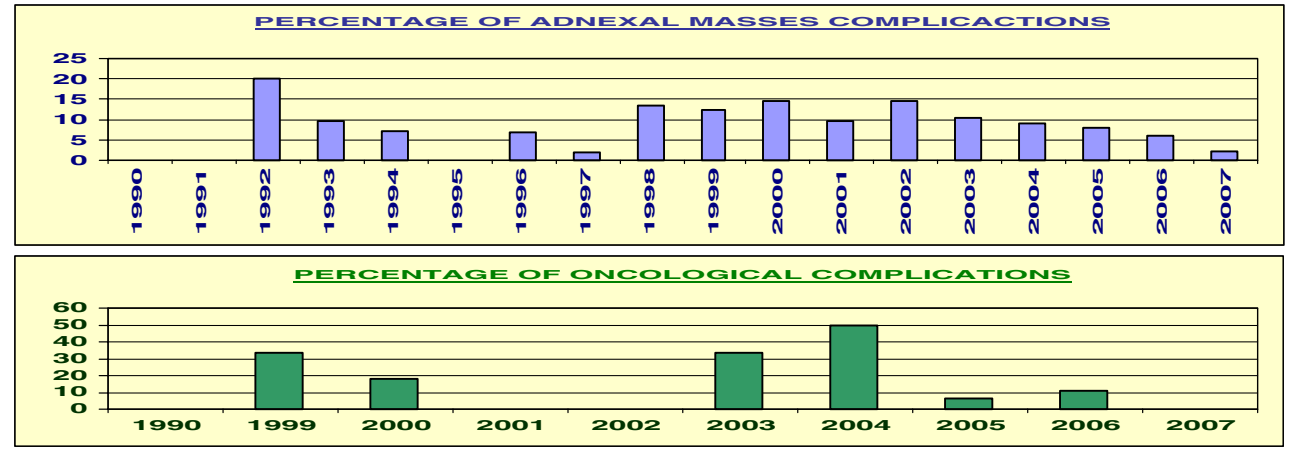

Conclusion: Global department laparoscopic complication rate is firmly related to learning curve and it will be modified when introducing new procedures or new surgeons.

\section{FC_71}

\section{Estimation of level of difficulty in hysterectomy; results} from a randomised controlled clinical trial B. Nieboer ${ }^{1}$, M. Bongers ${ }^{2}$, M. Spaanderman ${ }^{1}, K$. Kluivers ${ }^{1}$ ${ }^{I}$ Radboud University Medical Centre, Nijmegen, Netherlands, ${ }^{2}$ Maxima Medical Centre, Veldhoven, Netherlands

Objective: To measure the accuracy of the assessment of the difficulty of hysterectomy (abdominal and laparoscopic). Both an overestimation and underestimation of the difficulty of surgery are detrimental, since they can lead to time stress in theatre and unoccupied operating rooms, respectively.

Materials and methods: In a randomized study on laparoscopic (LH) versus abdominal hysterectomy (AH), the gynecological surgeons were asked to indicate the expected level of difficulty (preoperatively) and the experienced level of difficulty (postoperatively) on a 1-10 Visual Analogue Scale (VAS). A difference between expected and experienced level of three points or more was considered clinically relevant. Statistical analysis was performed using SPSS 14.0. $P<0.05$ was considered significant.

Results: In 72 out of 76 randomized patients, the pre- and postoperative VAS scores were both available. The expected and experienced level of difficulty was associated with both the weight of the patient en the size of the uterus on vaginal palpation. In 13 cases $(18 \%)$ the difference in VAS scores was three items or more ( $\triangle$ VAS $\geq$ $3)$. These clinically relevant differences were equally divided between the groups ( 6 in $\mathrm{LH}$, seven in $\mathrm{AH}$ ), and 11 were positive (i.e. surgery was experienced more difficult as expected). In case of uterine malignancy, an underestimation of the difficulty was more likely as compared with the benign indications $(p=0.005)$. LH was expected (4.8 vs $3.4 ; p=0.011$ ) and experienced (4.3 vs $2.9 ; p=$ 0.017 ) as more difficult compared with $\mathrm{AH}$.

Conclusion: An accurate assessment of the difficulty of surgery is important for adequate planning of surgery, thus avoiding stress in theatre and poor resource use. In the present study, a clinically relevant discrepancy between the estimated and experienced difficulty of hysterectomy occurred in $18 \%$ of cases, the majority being an underestimation. LH was generally expected and experienced as a more difficult operation compared with $\mathrm{AH}$.

\section{FC_72}

National Inpatient Diagnostic Hysteroscopy survey S. Tawfeek, P. Scott Derriford Hospital, Plymouth, UK

Objectives: This survey was undertaken to determine the practice patterns of how inpatient hysteroscopy is carried out in the UK. Also, we were aiming to assess the operators understanding of the hysteroscope optics and how this should reflect on the technique used. We believe this will help to set up a consensus, reduce complication and develop a national guideline in accordance with the way we undertake this procedure.

Materials and methods: Postal questionnaires were sent within the first week of June 2007 to 700 consultant gynaecologists in the UK. The surveyed gynaecologists were selected from the RCOG register of Fellows and Members. The questionnaire was included questions regarding preoperative preparation, operative techniques, post operative management and follow up. 
Results: Three hundred twenty-six (46.5\%) responses were returned; $303(43.2 \%)$ consultant gynaecologists completed the survey and $23(3.3 \%)$ incomplete questionnaires were retuned by consultants who currently do not undertake hysteroscopies. It appears that there is moderate consensus in with the way we undertake this procedure. However, the results showed a limited understanding and misconception of hysteroscope optics and how that relates to surgical techniques used.

Conclusions: There was a clear lack of understanding of the optics of hysteroscopic procedure between gynaecologists. Optics related surgical techniques should be included into training programme. Professional societies are able to develop a general consensus for this procedure.

\section{FC_73}

Reproducibility of saline infusion sonography $\underline{\text { S. Beemsterboer }}^{I}$, A. Thurkow ${ }^{2}$, R. Verstraeten ${ }^{1}$, H. Brolmann ${ }^{1}$ ${ }^{I} V U$ University Medical Center, Amsterdam, Netherlands, ${ }^{2}$ St. Lucas-Andreas Hospital, Amsterdam, Netherlands

The objective of this study was to calculate inter- and intraobserver agreement divided by different levels of experience. The inter-and intraobserver agreement was measured by scoring video material of 16 SIS procedures of women with abnormal uterine bleeding. Twenty-two observers were allocated to three different groups according to their level of experience. Kappa values were used to measure the inter- and intraobserver agreement. When looking at inter-observer agreement according to the different levels of experience there was a significant difference in Kappa values between experienced and less experienced observers. The group with the highest level of experience (group A) reached a mean Kappa value of 0.62 (95\% CI 0.56-0.67). Group B reached a Kappa value of 0.38 (95\% CI 0.33-0.43) and group C 0.47 (95\% CI 0.43-0.52). The inter-observer agreement in Group A is significantly higher compared to group $\mathrm{B}$ and $\mathrm{C}(p<0.001$ and $p=0.023$ resp.) whereas group $\mathrm{C}$ performs better compared to group B $(p=0.024)$. The results of intra-observer agreement $(n=7)$ show a good agreement expressed by a mean Kappa value of 0.66 (category A: 0.63 , category C: 0.71 ) We conclude that the inter-observer agreement in inexperienced sonographers is poor, while the intra-observer agreement is good. Therefore we consider the poor inter-observer agreement to be due to non-uniform diagnostic criteria. Uniform diagnostic criteria of SIS should be incorporated in the training of residents and other physicians performing these examinations.

\section{FC_74}

Localization of Essure ${ }^{\circledR}$ microinserts using 3D ultrasound after hysteroscopic sterilization. A prospective single-center study

G. Bader, S. Boustany, J.-P. Bernard, B. Guyot,

A. Fauconnier, Y. Ville

Poissy Hospital, Poissy, France

Objective: To evaluate the ability of $3 \mathrm{D}$ ultrasound for the localization of Essure ${ }^{\circledR}$ micro-inserts after hysteroscopic sterilization.
Materials and methods: Prospective single-center study. Between April 2006 and January 2008, all patients undergoing ambulatory hysteroscopic sterilization were assessed using 3D Transvaginal ultrasound and plain pelvic X-ray 3 months after surgery. 46 patients were also evaluated using 3D ultrasound immediately after the procedure. Three positions of the microinserts crossing the utero-tubal junction were described by $3 \mathrm{D}$ ultrasound: ((I, II, III) ideal position. (I, II) proximal position. (II, III) distal position).

Results: During the study period, 84 patients were included. The mean age was 41.4 years (range, $30-48$ years). All the procedures were ambulatory. Eight procedures $(9.5 \%)$ and 76 procedures (94\%) were performed with and without general anaesthesia (Bettochi) respectively. All procedures were uneventful. Bilateral tubal placement of the micro-inserts was successful in 79 patients (94\%). The mean operative time was $11.3 \mathrm{~min}$ (range, 6-20 min). In case of failure, laparoscopic tubal ligation was systematically proposed. The mean number of coils visualised by hysteroscopy was 4.4 (range, 2-9) on the right side and 3.9 on the left (range, 18). Transvaginal 3D ultrasound imaging successfully demonstrated adequate placement of the micro-inserts within the uterine mucosal and cornual portion to the proximal fallopian tube. On the right side, immediate 3D ultrasound showed position (I, II, III) and position (II, III) in 26 cases (57\%) and 20 cases (43\%) respectively. On the left side, immediate $3 \mathrm{D}$ ultrasound showed position (I, II, III) and position (II, III) in 28 (60\%) and 18 patients (40\%) respectively. 3D ultrasound performed 3 months after surgery showed coils position (I, II, III) and (II, III) in 44 cases (58\%) and 32 cases (42\%) respectively on the right side and position (I, II, III) and (II, III) in 41 cases (54\%) and 35 cases (46\%) respectively on the left. Positions I (intra-uterine device) and III (distal fallopian tube or intraperitoneal position) were not found in our patients. Coils position at days 0 and 90 was the same in all cases. No intra-uterine or intra-peritoneal implant migration was observed in our study. Radiologic assessment using plain pelvic X-ray performed 3 months after surgery confirmed the intrapelvic placement of the implants without further description. No pregnancy was reported in our series since the beginning of our experience.

Conclusion: Transvaginal 3D ultrasound performed either immediately or 3 months after hysteroscopic sterilization could confirm adequate Essure ${ }^{\circledR}$ micro-inserts localization. Further studies are needed to assess whether immediate 3D ultrasound would definitely assess appropriate localization of the implants when hysteroscopic placement is adequate.

\section{FC_75}

Improvement of the Essure ${ }^{\circledR}$ inserting system: ESS305 versus ESS205, a clinical comparison in an outpatient setting

C. Janssen

Groene Hart Hospital, Gouda, Netherlands

Objective: To assess whether the newly developed Essure ${ }^{\circledR}$ inserting system ESS305 is advantageous in comparison to the previously developed ESS205 with respect to the ease of use, translated in duration of the insertion, patient's pain scores, 
problems with release and detachment of the implants and ease of use in general according to nurses and physicians.

Materials and methods: The data of two patient cohorts, one treated with ESS205 ( $n=45)$ and the other treated with ESS305 ( $n$ $=41$ ) in consecutive periods (July 2007 to December 2007and December 2007 to April 2008 respectively) were compared. All treatments were performed at the outpatient clinic with a Bettocchi like, vaginoscopic approach by two experienced physicians. The duration of treatment was defined as the time from introduction of the hysteroscope into the patient until the moment of it's withdrawal. The pain was scored by the patient on a visual analogue scale (VAS) immediately after treatment. The subjective assessment of nurses and physicians was obtained by interview.

Results: The mean duration of treatment was $8.1 \mathrm{~min}$ in the ESS205 group and $6.6 \mathrm{~min}$ in the ESS305 group. This difference was not statistically significant ( $p=0.12$, two-tailed $t$-test). The VAS scores were 4.1 and 3.4 respectively and this difference was also not statistically significant ( $p=0.22$, two-tailed $t$-test). Problems with release and detachment occurred five times in the ESS205 group, but never with the new device (ESS305), which was appraised positively by nurses and physicians.

Conclusions: The newly developed Essure ${ }^{\circledR}$ device (ESS305) was supposed to have several advantages in comparison with the old one (ESS205), with respect to ease of use, such as easier release and detachment, better visible markers and an anti-splash valve. Problems with release and detachment, which incidentally had caused dramatic trouble and increase of duration of treatment with the ESS205, indeed did not occur with the new device. The mean duration of session was shorter and the VAS score lower with the new device, but both not statistically significant. The new inserter sleeve, with a valve to prevent splashing back fluid during treatment and which cannot rupture anymore, was also appreciated very much by the nurses and physicians.

\section{FC_76}

Relationship between the IUD and the placement success rate of Essure ${ }^{\circledR}$ device

J.E. Arjona Berral, E. Velasco Sánchez, E. Sainz de Vicuña Sainz, B. Hinojosa Marín, B. Pelegrín Sánchez

Hospital Universitario Reina Sofia, Córdoba, Spain

Objective(s): To study the relationship between the use of IUD and the failure rate of $\mathrm{ESSURE}^{\circledR}$ devices placement.

Materials and methods: Prospective study of 2,849 women, in which 314 were users of IUD; in 207 women, the IUD was removed before sterilization, and in 107 , the tubal microinserts were tried to place with the IUD inside.

Results: The failure rate in our unit is $1 \%$. In users of IUD, the rate increase till $4.8 \%$. When we study separately users of IUD, the rate increase till $4.8 \% ; 3.8 \%$ for women with IUD removed before sterilization, and $6.5 \%$ if we tried to place microinserts with IUD inside.

Conclusions: The use of IUD as a contraception method, or the placement of the Essure ${ }^{\circledR}$ intratubal devices in a patient with a IUD, increase the failure rate of hysteroscopic sterilization.
FC_77

Pregnancy after Essure ${ }^{\circledR}$ sterilisation: eight pregnancies in the Netherlands

L. Moolenaar, S. Veersema

St. Antonius ziekenhuis, Nieuwegein, Netherlands

Objective: To report eight unintended cases of pregnancy after hysteroscopic sterilisation with Essure ${ }^{\circledR}$.

Materials and methods: A retrospective study was conducted to identify all reported pregnancies after Essure ${ }^{\circledR}$ sterilisation in the Netherlands in the period between 2002 and 2007.

Results: In 40 hospitals in the Netherlands an estimation of 4,000 Essure $^{\circledR}$ sterilisations were performed since the introduction in 2002. Eight cases of pregnancies were reported. Patients became pregnant after a mean of 6.4 months (range 1-21 months) after Ultrasound or Hysterosalpingography conformation of Essure ${ }^{\circledR}$ placement. Four women had ongoing pregnancies without complications, the other four women underwent a termination of pregnancy. In three cases unilateral placement was performed, the opposite tube appeared to be occluded with hysterosalpingography. In two cases bilateral placement of the micro-inserts was confirmed by ultrasonography. In retrospect there was one complete expulsion and one perforation. One case was due to misreading of the hysterosalpingography. In one case the patient was lost to follow-up after diagnosis of unsuccessful sterilisation. And in one case the cause of the failed sterilisation is unknown because a lack of information.

Conclusions: A total of eight pregnancies occurred, in four cases there was an incorrect position, in three cases unilateral placement was performed and one case is unknown. Unilateral placement where the opposite tube appears to be occluded with HSG can not be accepted as adequate sterilisation. Misinterpretation of the confirmation-test is another cause of unreliable sterilization. The presence of Essure ${ }^{\circledR}$ micro-inserts in the fallopian tubes appears not to interfere with successful outcome of pregnancy.

\section{FC_78}

Feasibility of NovaSure ${ }^{\circledR}$ endometrial ablation followed by Essure ${ }^{\circledR}$ hysteroscopic sterilization and follow-up by hysterosalpingography

R. Detollenaere, H. van Eijndhoven

Isala Klinieken, Zwolle, Netherlands

Objective: To evaluate the feasibility and results of Essure ${ }^{\circledR}$ hysteroscopic sterilization combined with NovaSure ${ }^{\circledR}$ global endometrial ablation (GEA).

Materials and methods: Eleven women (aged 32-45 years) with menorraghia underwent radiofrequency GEA immediately followed by Essure ${ }^{\circledR}$ hysteroscopic sterilization. All procedures were performed under general anaesthesia and at various times in the menstrual cycle. The endometrium was not pretreated by progesterone. Patients returned after 1 month for ultrasound evaluation and after 3 months for hysterosalpingography (HSG).

Results: Bilateral placement of the micro-inserts after GEA was successful in ten patients and unilateral placement in one patient 
with a former tubectomy. Mean procedure time was $10.2 \pm 4.2$ (5$20 \mathrm{~min}$ ), all tubal ostia were easily identified, no complications occurred per- or postoperatively and all patients were dismissed the same day. Transvaginal ultrasound after 1 month revealed proper localization for all micro-inserts. Routine follow-up HSG after 3 months was performed in ten of these patients. Bilateral tubal occlusion was documented in eight patients $(80 \%)$. In two cases $(20 \%)$ this was not possible because HSG only demonstrated minimal contrast medium in the uterine cavity. The latter was probably caused by severe uterine synechiae. All patients were satisfied with the result of the GEA, eight women reported amenorrhea, the others a strong reduction in menstrual blood loss. Conclusion: The combination of an Essure ${ }^{\circledR}$ hysteroscopic sterilization and NovaSure ${ }^{\circledR}$ GEA is feasible and safe. However, synechiae could affect the ability of documenting tubal occlusion by HSG. Whether or not HSG can be replaced by transvaginal ultrasound in this combined approach remains to be established.

\section{FC_79}

Effects of cost and social security co participation in the use of hysteroscopic tubal sterilization

A. Fradique

H dos Lusiadas, Lisbon, Portugal

Objectives: To study the effect of social security co-participation in the cost of hysteroscopic tubal sterilization in Portugal.

Materials and methods: The cost of a hysteroscopic tubal sterilization are over $1,000 €$, mainly due to the cost of the inserts. Till February, 2006, this cost was totally supported by the patient or the institution (in the case of state hospitals). After this date social security fully pay this procedure when done in a state hospital. We compare the number of procedures performed in each of the three regions of our country in the 2 years before (Group A) and after the implementation of this payment (Group B), in state hospitals. We try also to evaluate the evolution in the number of gynecologists performing the procedure and other factors that influence the choice of this procedure.

Results: When we compare the total number of procedures in the country we notice an increment of $2.5 x_{\mathrm{s}}$, meaning an augmentation of $253 \%$ (from 253 to 650 ). By regions the number of procedures increased $159 \%$ (from 186 to 277) in the North, $2,088 \%$ (from nine to 188 ) in the Center, and $284 \%$ (from 58 to $165)$ in the South region. Also the number of gynecologists performing the procedure duplicate (from 26 to 53), but only 21 have performed more than ten procedures.

Conclusions: The augmentation in the number of procedures, especially in region of the country where it was rarely done, after the start of social security payment, shows that cost is one of the important factors in the choice of sterilization technique. However, being hysteroscopic sterilization the easiest and safest technique for female sterilization, at present, it is far from being the first choice procedure. This means that other factors are involved. In our opinion, the small number of gynecologists trained to do the procedure and the lack of awareness for the advantages of this procedure among health workers and the general population, are the two others main causes that need to be corrected.
FC_80

UK experience of the Essure ${ }^{\circledR}$ procedure: highly effective, but can its uptake be increased?

N. Baxter, A. Baxter, M. Connor, V. Brown

The Royal Hallamshire Hospital, Sheffield, UK

Introduction: The Essure ${ }^{\circledR}$ hysteroscopic sterilisation procedure has been performed at the Royal Hallamshire Hospital, Sheffield since 2002. Our excellent experience reflects that of other centres in the world, however, uptake in the UK has been poor. This abstract discusses the issues.

Materials and methods: Sheffield has been one of the few centres in the UK to undertake the Essure ${ }^{\circledR}$ procedure in to any significant extent. We have also held regular training courses over the years. We have compared our results against those from other centres. In an attempt to make a business case to cover the expensive disposable cost of the Essure ${ }^{\circledR}$ procedure we have obtained an assessment from the York Health Economics Consortium.

Results: Since 2002 we have performed 39 procedures with the original Essure ${ }^{\circledR}$ device, 150 with the second generation and more recently five with the third generation device. All procedures have been performed in the outpatient department. Local anaesthetic has been used when necessary and for the last 2 years a vaginoscopic technique has been used. Our insertion success rate was poor (82\%) with the original devices, perhaps due to the learning curves of the three surgeons involved. After we moved on to the second generation device the insertion rate has been $96 \%$ and the 3/12 X-ray has been satisfactory in all women who attended for follow-up. Five women have failed to attend X-ray follow-up appointments. We have had one pregnancy after Essure ${ }^{\circledR}$ _ due to the procedure being performed in the luteal phase. Patient satisfaction rates are high at $98 \%$.

Discussion: Our experience has been excellent, but generally uptake for Essure ${ }^{\circledR}$ in the UK is low, largely due to the cost of the disposable. Although the cost to society and the NHS of a woman having a laparoscopic sterilisation is higher, the inflexibility of the tariff system does not cover the department's cost of expensive disposables. Cost effectiveness data exists to support the viability of hysteroscopic sterilisation. In this abstract we will present QUALY data on laparoscopic and hysteroscopic sterilisation and discuss ways to circumvent the funding issues that currently exist in the UK.

\section{FC_81}

Hysteroscopic tubal sterilization: a cost-effective procedure on an outpatient basis

M. Franchini ${ }^{1}$, F. Calonaci ${ }^{2}$, G. Lippi ${ }^{1}$, S. Calzolari ${ }^{1}$, L. Cianferoni ${ }^{1}$, P. Florio ${ }^{2}$

${ }^{1}$ Palagi Freestanding, Firenze, Italy, ${ }^{2}$ Dep Gynecology, Siena, Italy

Study objective: To compare the health, non-health and indirect cost of permanent female sterilization by Essure ${ }^{\circledR}$ hysteroscopic sterilization and laparoscopic bilateral coagulation on an inpatient or outpatient basis

Design: Cost-minimisation analysis

Setting: Department of gynaecology at a general hospital 
Patients: Sixty women of reproductive age seeking permanent birth control

Interventions: Hysteroscopic placement of Essure ${ }^{\circledR}$ device, in an operating theatre, with either general anaesthesia or intravenous sedation for the first 25 procedures and in an office setting without either intravenous sedation or local anaesthesia for the second 31 cases. Laparoscopic tubal sterilization, in an operating room, using bipolar forceps according to standard techniques with general anaesthesia in a day surgery setting

Measurements and main results: The various costs associated with the two procedures in a day surgery setting or on an outpatient basis were compiled by activity-based costing, an advanced accounting system that allocates resource costs to products based on activity consumption. The hysteroscopic sterilization had greater cost compared with laparoscopic tubal sterilization when both procedures were performed in an operating room setting. Essure ${ }^{\mathbb{R}}$ procedure had higher costs for disposable equipment. A significantly decreased in costs was detected in performing hysteroscopic sterilization on an outpatient basis. The decrease per patient in institutional cost from a day surgery setting to an outpatient basis was euros 380 because in a day surgery setting the majority of the cost was related to hospital costs (operating room, recovery room, pharmacy).

Conclusion: In our institution, the Essure ${ }^{\circledR}$ hysteroscopic sterilization on an out patient basis had significant cost savings even if the Essure ${ }^{\circledR}$ procedure had higher costs for disposable equipment.

\section{FC_82}

Hysteroscopic tubal sterilization (Essure ${ }^{\circledR}$ ) in women with an intrauterine device

A. Agostini, M. Petrakian, J. P. Estrade

Obstetrics And Gynecology Department, Marseille, France

Introduction: The aim of this study is to evaluate the feasibility and effectiveness of hysteroscopic tubal sterilization in women with an intrauterine device (IUD).

Materials and methods: Prospective observational study of hysteroscopic tubal sterilization in women with IUD. Feasibility of the procedure, localization of microinserts and tubal occlusion evaluated by hysterography.

Results: Hysteroscopic tubal sterilization was performed in six patients. Hysterography showed correct placement of microinserts and bilateral tubal occlusion in all patients.

Discussion: Hysteroscopic tubal sterilization in women with an IUD appears to be a feasible and effective procedure.

\section{FC_83}

Reliability of a continuous pain score meter: real time pain measurement

E. Boormans ${ }^{1}$, P.van Kesteren ${ }^{1}$, R. Perez ${ }^{2}$, H. Brolmann ${ }^{3}$,

W. Zuurmond ${ }^{2}$

${ }^{I}$ Department of Obstetrics and Gynaecology, Onze Lieve Vrouwe Gasthuis, Amsterdam, Netherlands, ${ }^{2}$ Department of anaesthesiology, $V U$ medical center, Amsterdam,

Netherlands, ${ }^{3}$ Department of Obstetrics and Gynaecology, VU medical Center, Amsterdam, Netherlands
Introduction: Patient acceptability for outpatient procedures is usually assessed by measuring pain using a Visual Analogue Score (VAS). The disadvantage is that VAS is a once-only measurement and doesn't give a continuous impression of the patient's pain perception throughout the procedure. We have, therefore, developed a continuous pain score meter (CPSM) that electronically measures pain score in a continuous instead of a single manner.

Materials and methods: We have determined the test retest reliability of this meter. Thirty-two healthy volunteers received a reproducible pain stimulus on the right thumbnail and forearm. After a 1-min interval the procedure was repeated. Pain was continuously measured with the CPSM, providing values of peak continuous pain score (peak CPS) and area under the continuous pain score curve (AUC CPS).

Results: The intra class correlation coefficient (ICC) and the $95 \%$ limits of agreement were used to assess the agreement between measurements. ICC of peak CPS (0.89 and 0.83) and AUC CPS (0.79 and 0.86$)$ for nail and arm showed a good reproducibility.

Conclusion: We have successfully established the test retest reliability of a newly developed pain score meter.

\section{FC_84}

A new approach to vaginal top closure after total laparoscopic hysterectomies (TLH)

${\underline{\text { J. Rhemrev }^{1}}}^{1}$, M. Smeets ${ }^{1}$, F.W. Jansen ${ }^{2}$

${ }^{I}$ Bronovo Hospital, The Hague, Netherlands, ${ }^{2}$ Leids Universitair Medisch Centrum, Leiden, Netherlands

Introduction: Although the incidence of vaginal cuff dehiscence after different modes of hysterectomies is low $(0.1-4.93 \%)$, it is a serious complication. It occurs 40-50 times more often after TLH in comparison with total vaginal - and total abdominal hysterectomies. The purpose of this study is to compare the incidence of vaginal cuff dehiscence after two different closure techniques of the vaginal cuff after TLH.

Materials and methods: The study has been designed as an observational case study. During the period 2003-2008 a cohort of patients $(n=200)$ were included from two teaching hospitals. All patients had the same surgical procedure for the TLH. Vaginal cuff closure was performed by either a vaginal-or a laparoscopic approach. The laparoscopic technique consisted of a running suture fixed with two clips. Incidence of complications were determined and compared in the two groups.

Results: No significant differences in vaginal cuff dehiscence were seen between the two groups $(p<0.05)$. Neither did we determine a significant difference in occurrence of other known complications i.e. infections, post operative haematomas and abscess formation.

Discussion: Laparoscopic closure of the vaginal cuff seems to be a safe procedure. No significant differences could be seen comparing the two techniques. Eventually the laparoscopic suturing technique takes significant less time to complete than the vaginal suturing procedure. Moreover in selected groups of patients such as obese individuals and those with a poor descend of the uterus the laparoscopic technique should be the first 
choice procedure. Altogether because the laparoscopic technique seems to be less traumatic and less complicated to perform. Finally this laparoscopic suturing technique was also applied as a modified Mac Call suture to prevent patients from future vaginal prolapse.

\section{FC_86}

Laparoscopic sacral colpopexy: learning curve C.Youssef $^{l}$, D. Dautin ${ }^{1}$, J. Nassif ${ }^{2}$, A. Wattiez ${ }^{2}$

${ }^{1}$ Hautepierre University Hospital, Strasbourg, Bas Rhin, France, ${ }^{2}$ IRCAD/EITS, Strsbourg, Bas Rhin, France

Objective: At 80 years old, $11 \%$ of women had undergone prolapse surgery. $29 \%$ of these patients are operated more than once. Conventional vaginal surgery presents a high rate of recurrence $(30 \%)$. Prosthetic replacement predispose to a high risk of retraction $(30 \%)$ and exposure (11\%). Sacrocolpopexy is still the gold standard cure nowadays. Its drawbacks are its difficulty and lack of reproducibility. The aim of our work was to study the learning curve of a senior laparoscopic surgeon acquiring this technique.

Materials and methods: We recruited 56 patients were operated for genital prolapse by laparoscopic sacro-colpopexy.35 by the learning surgeon (L) and 21 by an expert (E) one. The technique is to place two meshes: the first, anterior, inter-vesicovaginal fixed on the anterior vaginal wall and the second, posterior, inter-rectovaginal fixed on pubo-rectalis muscle. Both meshes are fixed to each other at the level of the uterine isthmus. The posterior one is then sutured to the promontory. We compared retrospectively for both surgeons (L) and (E): patient's characteristics, duration and conduct of interventions, post operative follow up and the anatomical result at one and 3 months. The learning curve of the surgeon (L) was realised by reporting the duration of each procedure to its range.

Results: Patients' mean age is 55 and 58 years, BMI: 23.7 and 25.6, Hemoglobin gradient: 1.53 and $1.24 \mathrm{~g}$ for surgeon (L) and (E) respectively. The two groups are statistically comparable for prolapse grade, operative and post-operative complications and anatomical results at 1 and 3 months. Mean operating time for surgeon (L) was marked by a steady decline. It went from 360 to $200 \mathrm{~min}$. After 25 procedures, the durations of interventions were included in the average described in the literature: $180 \pm$ $50 \mathrm{~min}$ in about $88 \%$ of cases. The mean operating time for the surgeon (E) was constant: $120 \mathrm{~min}$.

Conclusion: A minimum of 25 sacracolpopexies is necessary to be performed in order to reach an acceptable operative time as cited in published data. Post operative complications rate seem not to be influenced by surgeon's expertise.

\section{FC_ 87}

\author{
Assessment of manual laparoscopic skills with the giessen \\ bench station-training \\ J. Ionesi-Pasacica, A. Hackethal, A. Döring, \\ H.-R. Tinneberg \\ Universitäts-Frauenklinik, Giessen, Hessen, Germany
}

Introduction: There is a great need to evaluate manual laparoscopic skills in surgery. So far it is not feasible. In need for accreditation of endoscopic courses, we designed an easy to repeat bench station training consisting of four tasks and a standardized score sheet.

Materials and methods: The Giessen Bench Station-Training (GBS-Training) is based on a four task training session. The first task consists of four peas needing to be placed on golf teas. Second, a needle has to be manoeuvred through eyelids varying in size. The third task consists of cutting a defined, triangle figure out of an examination glove. For the fourth task the participants were asked to perform an interrupted suture with a safety sling. At the first day and day six, the last course day, the participants performed the identical GBS-Test. These two performances were videotaped and later analysed by trainers and experts using a 61 item defined criteria form. Results: Using defined criteria for the four task bench model it is possible to differentiate between novices and advanced surgeons as well as experts. It is furthermore possible to measure the improvement of technical skill over the examined specific time period, for example 6 days relating to the Giessen Endoscopic Workshop. As an example, the results referring to the first task, pea placement: How many tries where needed to grab the four peas out of the bucket? (Pre-test 7.3 tries; Post-test 4.9 tries $=\varnothing 33 \%$ improvement. Time needed to complete the first task? (Pre-test $240.65 \mathrm{sec}$, Post-test $150 \mathrm{sec}=\varnothing 30 \%$ improvement). How many peas have been lost unintentionally from the instruments during movement? (Pre-test 2.4 peas, Post-test 0.91 peas $=\varnothing$ $62 \%$ improvement).

Discussion: Technical laparoscopic skills can be evaluated using defined assessment criteria with a standardized reproductable skills assessment device. Although the participants had varying laparoscopic experiences, individual training benefits after attending a laparoscopic course at the Giessen School of Endoscopic Surgery was assessable.

\section{FC 88}

Which is the best type of laparoscopic hysterectomy (LH)? N. Ahmed-Ebbiary, A. Karim, P. Thean

East Lancs Hospitals, Lancashire, UK

Introduction: Laparoscopic-assisted vaginal (LAVH) and total laparoscopic (TLH) hysterectomy with/without bilateral salpingooophorectomy ( \pm BSO) are well-established procedures. However, TLH is performed less frequently and the pros and cons of each are still debated.

Objective: Assess the effect of type of LH on various outcome measures.

Patients and design: Retrospective analysis of $\mathrm{LH}$ performed by three experienced gynaecologists, A, B and C, in order of seniority. The most recent consecutive 20 cases for each gynaecologist were selected.

Setting: District General Hospital.

Intervention: LAVH or TLH.

Outcome measures: Theatre time, blood loss/transfusion, return to theatre, length of stay (LOS) and long-term complications. 
Results: Gynaecologists A and B performed LAVH \pm BSO and were grouped together, while gynaecologist $\mathrm{C}$ exclusively performed TLH \pm BSO. Mean patients' age was 42.8 years, but TLH patients were significantly younger than those in the LAVH group (38.2 \pm 1.3 years vs. $45.0 \pm 1.2$ years, $P<0.003$ ). Most patients in both groups had LH for abnormal periods \pm pelvic pain but a higher proportion in the TLH group had associated extensive endometriosis/adhesions $(36 \%$ vs. $15 \%, P=\mathrm{NS})$. TLH was associated with significantly longer theatre time compared to LAVH $(200 \pm 7.0 \mathrm{~min}$ vs. $142 \pm 11 \mathrm{~min}, P<0.003)$. Blood transfusion was not required following TLH but was required in four patients $(10 \%)$, three of them $(8 \%)$ returned to theatre following LAVH. However, mean drop in postoperative $\mathrm{Hb}$ was similar in TLH and LAVH groups (2.3 gm vs. 2.7 gm, $P=\mathrm{NS}$ ). LOS was significantly shorter following TLH compared to LAVH ( $2.0 \pm 0.3$ days vs. $4.5 \pm$ 0.2 days, $P=0.0001)$. Long-term complications developed in one patient $(5 \%$, spotting) and five patients $(13 \%$, prolapse \pm SI, dsyparaunia) following TLH and LAVH, respectively.

Conclusions: TLH is known to be technically more difficult and higher proportion of severe endometriosis/adhesions was encountered, thus, the longer theatre time. However, TLH was associated with shorter LOS, thus, quicker recovery, less likelihood of blood loss, immediate/long-term postoperative complications. A larger study is suggested to establish the advantages, if any, of TLH over LAVH. This would help to counsel patients and arrange training for future gynaecologists accordingly.

*Institution where the study was performed.

\section{FC_89}

The results of treatment in patients with early cervical cancer managed by laparoscopy and conventional surgery P. Sobiczewski, M. Bidzinski

The Maria Sklodowska-Curie Memorial Cancer Center, Gynecologic Oncology Department, Warsaw, Poland

The objective of the current study was to compare the results of surgical treatment in pts with early cervical cancer managed by laparoscopy and laparotomy.

The goal was to evaluate and compare disease free survival (dfs) and recurrence rate in both groups.

Materials and methods: A retrospective analysis was carried out of 22 patients treated by laparoscopy between Jan 2001 and Dec 2004 and 58 patients treated by laparotomy between Jan 2001 and Dec 2002.The patients were in clinical stage of disease Ia, Ib1 and IIa. The radical hysterectomy with lymphadenectomy (Piver II) was performed by laparotomy in 58 pts and the Total Laparoscopic Radical Hysterectomy in 22 pts. Disease free survival (dfs) in both groups was evaluated with the Kaplan-Meier method and was compared using the log-rank test. The median follow-up in laparotomy group was 47 months and in laparoscopy group 26 months. Relapses occurred in seven patients managed by laparotomy group and in three patients after laparoscopy (in 2pts with intraperitoneal dissemination).

Dfs was $0.86( \pm 0.049)$ and $0.82( \pm 0.098)$ in both groups respectively.
There was no statistically significant difference in dfs and recurrence rate between laparotomy and laparoscopy groups. Conclusion: Laparoscopy may be used as alternative to conventional surgery, although the oncological safety of laparoscopic technique should be confirmed in a prospective study.

\section{FC_90}

From open radical hysterectomy to Robot assisted laparoscopic radical surgery for early stage cervical cancer: a single institution's learning curve

$\frac{\text { R. Zweemer }}{}{ }^{l}$, W. Marchien van $\mathrm{Baal}^{2}$, J. van de Lande ${ }^{3}$, J. Dijkstra $^{4}$, R. Verheijen ${ }^{1}$

${ }^{1}$ University Medical Centre Utrecht, Utrecht, Netherlands, ${ }^{2} \mathrm{VU}$ Medical Center, Amsterdam, Netherlands, ${ }^{3}$ Kennemer Gasthuis, Haarlem, Netherlands, ${ }^{4}$ St. Lucas Andreas Hospital, Amsterdam, Netherlands

Introduction: In radical surgery for early stage cervix cancer a Wertheim laparotomic procedure has been the gold standard for more than 100 years. The daVinci Robot system has now made a laparoscopic approach to this procedure feasible. Our study investigates whether this new approach is of benefit to the patient and whether acceptable operating times and efficiency can be achieved rapidly.

Materials and methods: Our single institution surgical team obtained experience with the Robot system in a training facility, consequently experience was broadened on pelvic lymphadenectomy and simple hysterectomy and subsequently we started to perform radical hysterectomies in September 2006. Before and after that date the last open procedures and the first Robot procedures by the same team were compared with respect to: Number of lymph nodes retrieved (as a marker for radicality of surgery), operating time, blood loss, hospital stay and complications.

Results: Fourteen open cases were compared to 13 Robot cases. The number of lymph nodes retrieved was 26 versus 32 . Blood loss in the open cases was always over 1,000 cc (median 2,000 cc) while in the Robot cases the median loss was $300 \mathrm{cc}$. Mean hospital stay was reduced by 5 days. The theatre time for the robot procedure was reduced from $9 \mathrm{~h}$ to less that $4 \mathrm{~h}$ in the course of our 13 cases and compared well to the 3 h 50 min mean theatre time for an open procedure. Two complications occurred in the open group and one in the Robot group.

Discussion: Robot assisted radical hysterectomy is of benefit to the patient with early stage cervical cancer by a reduction of blood loss and reduced hospital stay. A learning curve of less than less than 20 cases will reduce the operating time to a level comparable to open surgery.

\section{FC_91}

\author{
Laparoscopic treatment of adnexal masses in pediatric \\ and adolescent patients \\ Z. Stankovic, L. Andjelic \\ Mother and Child Health Institute of Serbia, Belgrade, Serbia
}


Objective: To assess the value of preoperative ultrasound examination in predicting the feasibility of laparoscopic surgery for benign adnexal masses in pediatric patients.

Materials and methods: Morphological assessment using Ueland's index and ovarian crescent sign (OCS) examination were evaluated for all patients who underwent surgical treatment for ovarian masses from January 2006 to March 2008. The ultrasound criteria used to select patients for laparoscopic surgery included: morphology index $\leq 6$ and morphology index $\geq 7$ with presence of OCS. Results: Fifty six girls (age range 6 months to 19 years), 16 of them premenarchal, were surgically treated for 60 ovarian masses. Based on ultrasound assessment, 40 patients were booked for laparoscopy and 16 for laparotomy. In all, 40 patients with 44 adnexal masses had their operation completed laparosopically, including eight masses with tumor volume $>500 \mathrm{~cm}^{3}$. On histological examination 47 masses were benign, including 19 torsions, and 13 were malignant. Laparoscopic ovarian cystectomy was successfully done in 42 of 44 adnexal masses. The preoperative ultrasound assessment predicted the positive outcome of laparoscopic surgery with a sensitivity of $95 \%$, specificity of $100 \%$, PPV of $100 \%$. Preoperative ultrasound assessment also predicted the success of laparoscopic ovarian cystectomy with a specificity of $87 \%$ and PPV of $95 \%$. Conclusion: Ultrasonographic assessment with morphological analysis and OCS is a helpful tool for assessing the feasibility of laparoscopic girls and adolescents with benign adnexal lesions. Laparoscopic ovarian preservation is possible for the most of benign adnexal masses.

Objective: To assess the value of preoperative ultrasound examination in predicting the feasibility of laparoscopic surgery for benign adnexal masses in pediatric patients.

Materials and methods: Morphological assessment using Ueland's index and ovarian crescent sign (OCS) examination were evaluated for all patients who underwent surgical treatment for ovarian masses from January 2006 to March 2008. The ultrasound criteria used to select patients for laparoscopic surgery included: morphology index $\leq 6$ and morphology index $\geq 7$ with presence of OCS. Laparoscopic surgery was classified as successful if the mass was removed completely without resorting to laparotomy.

Results: Fifty six girls (age range 6 months to 19 years), 16 of them premenarchal, were surgically treated for 60 ovarian masses. Based on ultrasound assessment, 40 patients were booked for laparoscopy and 16 for laparotomy. In all, 40 patients with 44 adnexal masses had their operation completed laparosopically, including eight masses with tumor volume $>500 \mathrm{~cm}^{3}$. On histological examination 47 masses were benign, including 19 torsions, and 13 were malignant, including one laparoscopic treated dermoid cyst with foci of immature teratoma. Laparoscopic ovarian cystectomy was successfully done in 42 of 44 adnexal masses, but with two salpingectomies. Laparoscopic adnexectomy, ovariectomy was performed each in a patient with adnexal torsion and sclerosing stromal tumor, respectively. The preoperative ultrasound assessment predicted the positive outcome of laparoscopic surgery with a sensitivity of $95 \%$, specificity of $100 \%$, PPV of $100 \%$. Preoperative ultrasound assessment also predicted the success of laparoscopic ovarian cystectomy with a specificity of $87 \%$ and PPV of $95 \%$.
Conclusion: Ultrasonographic assessment with morphological analysis and OCS is a helpful tool for assessing the feasibility of laparoscopic girls and adolescents with benign adnexal lesions. Ovarian preservation during laparoscopic treatment is possible for the most of benign ovarian tumors.

\section{FC 93}

Learning uterine artery embolization with a simulator B. McLucas

David Geffen School of Medicine at UCLA, Los Angeles, CA, USA

Abstract topic: Fibroids, diagnosis and treatment

Introduction: To assess the use of a simulator to teach endovascular skills to gynecologists

Materials and methods: Review of endovascular skill training for surgeons using simulators. Physicians may be evaluated before and after use of the simulator. Simulation has been used as a parameter to assess competency in other specialties.

Results: Gynecologists need training in catheter manipulation, Carm use, and embolization, all skills not learned in residency. These skills may now be learned and improved on a recently developed simulator designed to teach UAE. The simulator will be demonstrated.

Conclusion: UAE is a recognized alternative to surgery for patients suffering from uterine myomata. Gynecologists offer their patients thorough understanding of the disease and ability to manage the patient post procedure. Use of the simulator will allow gynecologists to learn UAE and eventually develop the skills and judgment necessary to perform UAE.

\section{FC_94}

Intrauterine adhesions (IUA): Has there been progress in understanding and treatment over the last 20 years? C. Panayotidis ${ }^{1}$, S. Weyers ${ }^{2}$, J.Bosteels ${ }^{3}$, B.Van Herendael ${ }^{4}$ ${ }^{I}$ Royal Oldham Hospital, department of obstetrics and gynaecology, Oldham, UK, ${ }^{2}$ Department of Obstetrics and gynaecology Ghent University Hospital, Ghent, Belgium, ${ }^{3}$ Department Obstetrics and Gynaecologie Imelda ziekenhuis Bonheiden, Bonheiden, Belgium, ${ }^{4}$ Obstetrics and gynaecology department ZNA Stuivenberg, Antwerp, Belgium

We review the current evidence about the treatment modalities of intrauterine adhesions (IUA) or Asherman's Syndrome (AS). The clinical practice changed significantly over the last 20 years with technological advances in hysteroscopy and imaging techniques. Systematic approach, audit and well structured research are mandatory in order to establish the best treatment for the individual needs of patients. We present an up to date and complete review about management of IUA. IUA or AS are rather uncommon findings in general gynaecological practice. Hysteroscopic treatment seems effective and safe for IUA. The referral to a tertiary centre will help to centralise the most difficult cases and create the opportunity to study more in detail the efficacy of each treatment modality and to compare the different treatment techniques. 


\section{FC 95}

Hyalobarrier ${ }^{\circledR}$, a hyaluronan adhesion prevention gel: systematic review and meta-analysis of safety results in randomized controlled trials

V. Mais ${ }^{1}$, M. Giuseppina Cirronis ${ }^{1}$, F. Torasso ${ }^{2}$, E. Cossu ${ }^{1}$, M. Peiretti ${ }^{1}$, G. Benedetto Melis ${ }^{1}$

${ }^{I}$ Division of Gynecology, Obstetrics and Pathophysiology of Human Reproduction, Department of Surgery, Maternal-Fetal Medicine and Imaging, University of Cagliari, Ospedale S. Giovanni di Dio, Via Osped, 09124 Cagliari, Italy, ${ }^{2}$ Fidia Advanced Biopolymers s.r.l.,Via Ponte della Fabbrica 3/B, 35031 Abano Terme (PD), Italy

Introduction: Among site-specific barriers used as adhesion prevention agents, a hyaluronan gel, Hyalobarrier ${ }^{\circledR}$, made of ACP $200^{\circledR}$, an auto-crosslinked ester of hyaluronic acid without any foreign bridge molecules, is available in the clinical surgical practice. This study is a systematic review and meta-analysis of the safety results (adverse events or AEs) derived from the use of Hyalobarrier ${ }^{\circledR}$ in gynaecologic surgery, either endoscopic or open, compared to the standard surgery treatment without adhesion prevention agent.

Materials and methods: We performed a systematic review of the AEs obtained from clinical records of 297 patients assessed for safety in five randomized, controlled, prospective clinical trials conducted in 11 different Italian clinical centers. Patients underwent surgery for different pathologies (e.g. uterine myoma, endometriosis, adhesions, endometrial polyps, uterine septa, intrauterine synechiae) and were randomly allocated intra-surgery to receive Hyalobarrier ${ }^{\circledR}(n=150)$ or standard surgery $(n=147)$. Nature, onset time, severity, duration, outcome and relationship to study device of all AEs were recorded from first surgery along the whole study (from 2 up to 12 months). Meta-analysis was performed and expressed as odds ratios with $95 \%$ confidence intervals and $p<0.05$ was considered as statistically significant.

Results: A total of 391 AEs occurred in 185 patients. In the gel group 102 patients reported 215 AEs and in the control group 83 patients experienced 176 AEs. Most of AEs were classified as mild or moderate and occurred in the postoperative period in both groups. Seven patients in the Hyalobarrier ${ }^{\mathbb{R}}$ group and six patients in the control group experienced at least one serious AE. The type and the incidence of serious AEs were similar in both groups and they were due to basal conditions or coexisting illnesses. There were no deaths in either group. The meta-analysis of the frequent AEs (e.g. fever, anaemia, dysmenorrhoea, vaginal haemorrhage and pelvic pain) showed that these AEs had similar distributions in both groups with no clinically significant differences.

Conclusions: The meta-analysis of these safety results clearly permits to consider Hyalobarrier ${ }^{\circledR}$ a safe anti-adhesion agent to be used in gynaecologic surgery.

\section{FC 96}

\author{
NovaSure ${ }^{\circledR}$ endometrial ablation under local \\ anaesthesia in a primary care setting \\ Helen Ludkin, Sian Jones, Anne Connolly \\ Bradford \& airedale PCT, Bradford, West Yorkshire., UK
}

Background: Endometrial ablation is increasingly being offered in place of hysterectomy. The second generation devices are ideal for use under local anaesthesia but uptake has been slow. In Bradford there is an outpatient hysteroscopy service set up in a diagnostic and treatment centre since 2005. This is staffed by a consultant, a nurse hysteroscopist and a GP hysteroscopist. The clinic offers both diagnostic and operative outpatient hysteroscopy. From July 2006 we have undertaken NovaSure ${ }^{\circledR}$ under local as an additional choice for the women.

Aims: To study whether NovaSure ${ }^{\circledR}$ endometrial ablation is feasible in a primary care setting under local anaesthesia and is safe and acceptable to women.

Materials and methods: Data collection on 60 women having NovaSure $^{\circledR}$ under local anaesthetic. The women are pre-medicated with oral tramadol, cyclizine and diclofenac $90 \mathrm{~min}$ before the treatment. Anaesthesia is with a four quadrant deep cervical block using mepivacaine. The cervix is dilated to hegar $8 / 9$ and the device inserted after a cavity integrity check. Rescue analgesia is available in the form of Entonox. Following treatment the women are monitored for $1 \mathrm{~h}$ before discharge home. They are reviewed at 3, 6 and 12 months.

Results: Fifty-five women completed a NovaSure ${ }^{\circledR}$ ablation. Mean pain score during the procedure was 4.8 and before discharge from the clinic was 0.8 . Only four woman needed rescue analgesia. The amenorrhoea/spotting rate at 12 months is $89 \%$. Ninety-seven percent of women are very satisfied with their results. Only one woman has not had relief of her menorrhagia and has needed a rollerball of the residual cavity. Ninety-seven percent would recommend it to a friend. One woman has needed admission to secondary care for pain control.

Conclusion: NovaSure ${ }^{\circledR}$ under local anaesthesia is feasible and safe in a primary care setting. Initial results are impressive with high satisfaction at 12 months.

\section{FC 97}

The COAT Trial-a randomised controlled trial comparing outpatient endometrial ablation techniques (NovaSure ${ }^{\circledR} \mathrm{TM}$

vs. Thermachoice $\left.{ }^{\circledR}{ }^{\mathrm{TM}}\right)$ : menstrual bleeding and quality of life outcomes

N. Samuel ${ }^{1}$, S. Malick ${ }^{2}$, J. Daniels ${ }^{3}$, L. Middleton ${ }^{4}$, J. Gupta ${ }^{5}$ J. Clark

${ }^{1}$ Birmingham Women's Hospital, Birmingham, UK, ${ }^{2}$ Birmingham Women's Hospital, Birmingham, UK, ${ }^{3}$ University of Birmingham Clinical Trials Unit, Birmingham, UK, ${ }^{4}$ University of Birmingham Clinical Trials Unit, Birmingham, UK, ${ }^{5}$ Birmingham Women's Hospital, Birmingham, UK, ${ }^{6}$ Birmingham Women's Hospital, Birmingham, UK

Introduction: Endometrial ablation using NovaSure ${ }^{\circledR T M}$ bipolar frequency impedance controlled ablation and Thermachoice ${ }^{\circledR}$ IIITM thermal balloon ablation have been shown to be feasible in an outpatient, local anaesthetic setting. We conducted an RCT to establish whether either ablative technique performed in an outpatient setting was more effective in the alleviation of symptoms of heavy menstrual bleeding and improving life quality. 
Materials and methods: Eighty-one consenting women over 25 years old, with heavy menstrual bleeding and no desire to retain fertility were randomised to NovaSure ${ }^{\circledR T M}(N=42)$ or Thermachoice ${ }^{\circledR \mathrm{TM}}(N=39)$. Women with uterine pathology, other than submucosal fibroids $<3 \mathrm{~cm}$, were excluded. Menstrual status was described on a 5-point scale (heavy, moderate, light, spotting and none). Health related quality of life (HRQL) was assessed using a symptom-specific scale (Shaw questionnaire) and generic instrument (5Q-ED). All clinical outcomes were measured at 3 months post-treatment.

Results: There was no significant difference in amenorrhea rates between ablative techniques [7 of $36(19 \%)$ of women who had Thermachoice $^{\circledR \mathrm{TM}}$ versus 12 of $36(33 \%)$ who had NovaSure ${ }^{\circledR \mathrm{TM}}$ (relative risk $0.4895 \%$ CI $0.16-1.42$ )]. Similarly there was no significant difference where "spotting" was included in the definition of amenorrhea, [15 of 36 (42\%) Thermachoice ${ }^{\circledR T M}$ versus 19 of $36(53 \%)$ NovaSure ${ }^{\circledR \mathrm{TM}}$ (relative risk 0.79 (95\% CI $0.48-1.29)$ ]. Subjective improvement was reported in 30 of 36 (83\%) women in the Thermachoice ${ }^{\circledR \mathrm{TM}}$ group and 33 of $36(92 \%)$ in the NovaSure ${ }^{\circledR T M}$ group $\left(\chi^{2} p=0.29\right)$. There was no significant difference in changes in HRQL from baseline on either the Shaw questionnaire (Thermachoice ${ }^{\circledR T M}$ mean 41.4 (sd 27.4) vs. NovaSure $^{\circledR \mathrm{TM}}$ mean 46.2 (sd 26.8); $P=0.5$ ) nor for EQ-5D (Thermachoice ${ }^{\circledR \mathrm{TM}}$ mean 0.13 (sd 0.30) vs. NovaSure ${ }^{\circledR \mathrm{TM}}$ mean 0.40 (sd 0.29); $P=0.9$ ).

Conclusion: There were no significance differences between Thermachoice $^{\circledR} I I I^{\mathrm{TM}}$ and NovaSure ${ }^{\circledR \mathrm{TM}}$ at 3 months post-treatment in terms of menstrual status or HRQL, although there was a trend towards improved clinical outcomes in the NovaSure ${ }^{\circledR \mathrm{TM}}$ treated women. Larger trials are required to confirm or refute this. In the absence of clear differences in clinical outcomes, other factors e.g. length of procedure, patient experience and pain, should be considered when deciding which outpatient ablative modality to use.

\section{FC 98}

\section{Endometriomas as a marker of more severe pelvic endometriosis

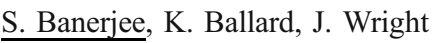 \\ Postgraduate Medical School, University of Surrey, Surrey, UK}

Introduction: Whilst superficial endometriosis can be treated effectively and safely by the majority of gynaecological surgeons, deep infiltrating disease (DIE) needs to be treated in specialist endometriosis centres. In order that women can be treated in the most appropriate setting, it is necessary to identify whether they have DIE. It has been established that there is a strong association between endometriomas and DIE. Endometriomas can be diagnosed with a high sensitivity and specificity by ultrasound examination, whereas other markers of endometriosis are more difficult to detect. This investigation looks at the value of endometriomas as a diagnostic marker of DIE.

Materials and methods: We collected prospective intra-operative data from 295 women with histologically confirmed endometriosis in order to investigate the role of endometriomas as a marker for
DIE, and in particular, recto-sigmoid disease. At operation all disease area were mapped and then excised and sent for histological confirmation of disease. Endometriomas were treated by stripping the capsule, histological confirmation of endometriosis being carried out in every case.

Results: Sixty-one women (21\%) were found to have an endometrioma. A significantly greater proportion of women with an endometrioma were found to have accompanying endometriotic disease affecting the bowel compared with women without an endometrioma $(77 \%$ versus $21 \% ; p<0.001)$. We also found a strong relationship between presence of endometrioma and posterior cul-de-sac obliteration, recto-sigmoid disease, and involvement of the sero-muscular layer of the bowel. The presence of an endometrioma significantly increased the probability of having recto-sigmoid disease, with a positive likelihood ratio of 6.96 (95\% CI; 4.04, 12.00). However, the absence of an endometrioma did not preclude recto-sigmoid disease, with a negative likelihood ratio of 0.55 (95\% CI; 0.45, 0.67). This makes the presence of an endometrioma a moderately useful test in terms of diagnostic accuracy.

Discussion: Whilst endometriomas provide a useful marker for DIE, the absence of an endometrioma does not preclude the presence of DIE, although a significantly lower proportion of women without an endometrioma will have DIE when compared with women with an endometrioma. The finding of an endometrioma together with other markers of endometriosis such as uterine fixity and palpable nodules in the posterior cul de sac may allow for a more sensitive and specific clinical test for DIE.

\section{FC_99}

A new narrow band imaging endoscopic system for the detection of surface pathology including endometriosis: a series of 95 patients

S. Chandakas $^{\text {I }}$, J. Erian ${ }^{1}$, E. Salamalekis ${ }^{2}$

${ }^{1}$ Iaso Group of Hospitals, Athens, Greece, ${ }^{2}$ Attikon

University Hospital, Athens, Greece

Introduction: The purpose of introducing optical electronics into video endoscopes is to improve the accuracy of diagnosis through image processing and digital technology. Narrow-band imaging (NBI), one of the most recent techniques, involves the use of interference filters to illuminate the target in narrowed red, green and blue $(\mathrm{R} / \mathrm{G} / \mathrm{B})$ bands of the spectrum. This results in different images at distinct levels of the mucosa and increases the contrast between the epithelial surface and the subjacent vascular network. NBI can be combined with magnifying endoscopy with an optical zoom. The aim of this new technique is to characterize the surface of the distinct types of gastrointestinal epithelia.

Materials and methods: A retrospective study in a university Hospital and a major gynaecological Hospital in Athens, Greece.95 women, mean age 33.9 with possible diagnosis of endometriosis, 58\% underwent surgery for fertility reasons.

Results: NBI was used with magnifying endoscopy to image and biopsy randomly selected areas in all 95 patients. A systematic image and a biopsy specimen evaluation process was followed, 
including unblinded assessment of an exploratory set of images and biopsy specimens, and blinded evaluation of learning and validation sets. Of the lesions, $82.7 \%$ were labelled as endometriosis, whereas only $55.9 \%$ of the patients were initially diagnosed with endometriotic lesions.

Discussion: NBI and other similar technologies provides an electronic, easier alternative to chromoendoscopy to aid the endoscopist in differentiation among benign, premalignant, and malignant mucosal patterns, as well as early stage endometriosis.

\section{FC_100}

\section{Primary bilateral tubal adenocarcinoma associated with unilateral tubal endometriosis: case report S. Carvalho, F. Pinto, S. Soares, R. Ramalho, V. Costa, N. Nunes, M. Rodrigues \\ Maternidade de Júlio Dinis, Porto, Portugal}

Introduction: Primary adenocarcinoma of the fallopian tube is the least common primary malignant tumor of the female genital tract. Bilaterality is also rare. Often the diagnosis is mistaken for ovarian tumor or tubo-ovarian mass. A case of bilateral primary tubal adenocarcinoma of serous type associated with tubal endometriosis is being reported.

Case report: Fifty-three year-old woman with primary infertility, hypertension, was admitted in our institution, with complaints of pelvic pain since 1 year. Her menstrual cycles were regular with normal flow. General physical and gynecological examination was unremarkable. Pelvic ultrasound showed a left sided complex adnexial mass with $6.0 \times 5.5 \mathrm{~cm}$, suggestive of endometrioma, uterus and right ovary were normal. Abdominopelvic tomography revealed an expansive left sided adnexial mass with cystic components and calcifications. Right ovary was normal with a milimetric calcification; uterus, bladder, liver were normal. Tumour markers Ca-125-42.20 U/ml. During exploratory laparotomy, left unilateral adnexal mass were identified, presumed to be of ovarian origin. Uterus, right tube and ovary were normal. Total abdominal hysterectomy, bilateral salpingo-oophorectomy, infracolic omentectomy were performed. Liver surface was normal, para aortic and pelvic lymph nodes wee not palpable and both para colic gutters were free. Post operative period was uneventful. Histologic examination showed a primary bilateral fallopian tube adenocarcinoma, stage (FIGO): IB, and unilateral adnexal endometriosis. Surgery was followed by chemotherapy.

Discussion: Primary carcinoma of fallopian tube is rare but extremely aggressive neoplasm. It is rarely suspected preoperatively. The symptom complex of "hydrops tubae profluence" said to be pathognomonic for this tumour is rarely encountered. Endometriosis is in some cases concomitant and can mistake the diagnosis in the first approach. The treatment is similar to that used for ovarian carcinoma and includes primary surgery comprised of total abdominal hysterectomy with bilateral salphingo-oopherectomy and staging followed by chemotherapy. Chemotherapy and irradiation are two adjuvant therapy that are similarly effective in fallopian tube carcinoma stage 1 and 2, with chemotherapy being preferred at the present time.

\section{FC 101}

Clue anatomical landmarks in pelvic lymphadenectomy A. Gorostiaga ${ }^{2}$ I. Villegas ${ }^{2}$, E. Beiro ${ }^{1}$, J. Cruz Quilez ${ }^{1}$, D. Andia $^{1}$, I. Brouard ${ }^{1}$

${ }^{1}$ Basurto Hospital, Bilbao, Spain, ${ }^{2}$ San Francisco Javier Hospital, Bilbao, Spain

Objective: To show the clue anatomical landmarks in pelvic oncologic surgery in cases of lymphadenectomy. Pelvic anatomy is not really as schematic as we imagine. Spaces are "virtual" and must be created and prepared by the surgeon, in order to make surgery easier. Paravesical and pararrectal spaces are the basic ones, the former delimitated by the external iliac artery, urinary bladder and the cardinal and umbilical ligament and the latter by the external iliac vessels, cardinal ligament and the posterior leaf of the broad ligament.

Materials and methods: Videosurgical demonstration of the dissection and preparation of the paravesical and pararrectal spaces, identification of the ureter and the clue vascular and nervous structures.

\section{FC 102}

Does endoscopic myomectomy help infertile patients?

B. G. Molnár, A. Fodor, A. Rónya, K. Áron

$\overline{\text { Dept. } O B / G Y N}$ University of Szeged, Szeged, Hungary

Objective: The aim of the study was to investigate the effect of hysteroscopic and laparoscopic myomectomy on success of infertility treatment.

Materials and methods: Fifty four infertile patients suffering from fibroids were involved in the study during a period of 2 years. Afterwards a previous check-up hysteroscopic or laparoscopic myomectomy was performed to ensure a regular shape uterine cavity and a normal size uterus. Patients were followed-up for 1 year to find out, whether the removal of fibroids can increase the pregnancy rate.

Results: The site of the fibroids was intramural in five cases, subserosal in 27 cases, mixed type in 11 cases and submucosal in 11 cases. The size of the fibroids ranged between 1 and $13 \mathrm{~cm}$. Thirtyfive of the cases were operated laparoscopicly, 15 hysteroscopicly and in four cases both hysteroscopy and laparoscopy was used to remove the fibroids. IVF-ET treatment was performed on 22 patients, artificial homolog insemination on five cases, the rest of the patients decided to conceive spontaneously. Ten of the 27 patients undergone assisted reproductive treatment became pregnant, seven of them during the first treated cycle, three of them during the third treatment, four of them already had four treatments but still did not conceive. Two pregnancies miscarriaged, three women had premature labour (two of them had twin pregnancies) and five delivered mature newborns. Two of the eight childbirth were spontaneous vaginal delivery, in six cases Caesarian Section was performed. Among those who tried to conceive spontaneously 18 became pregnant, but two of them had miscarriage.

Discussion: In case of laparoscopic removal of subserosal fibroids, diagnostic hysteroscopy has to be done previously. 
Removal of submucosal fibroids increases the effectiveness of assisted reproductive techniques.

\section{FC_103}

\section{Cryosurgery combined with phonophoresis in patients with tubal occlusive infertility and chronic salpingitis E. Hilkevich, E. Dubinskaya, E. Dovlethanova}

Scientific Center For Obstetrics, Gynaecology and Perinatology, Moscow, Russian Federation

The aim of the study was to improve treatment efficacy in patients with tubal occlusive infertility and chronic salpingitis.

Materials and methods: Three hundred patients with tubal occlusive infertility and chronic salpingitis were recruited and divided in to three groups. The first group (90 women) had proximal tubal occlusive infertility without pelvic adhesions, the second (160 women) - distal tubal occlusive infertility and pelvic adhesions at stage I-II, the third (50 patients) had distal tubal occlusive infertility and pelvic adhesions at stage III-IV.

Results: All patients of the first group were treated with cryosurgery combined with phonophoresis with antibacterial drugs for 5-6 days, which restored tubal patency in 34 (37.7\%) No side effects were reported. Clinical uterine pregnancy rate was $14.4 \%$, the live birth rate was $23.6 \%$, extrauterine pregnancy rate was registered in $3.3 \%$ (three women). In patients of the second and the third group laparoscopic surgery was performed combined with intraoperative Dextrans phonophoresis (5-10 sm distance of the peritoneum, frequency $26.5 \mathrm{kHz}$, duration $1-5 \mathrm{sec} / \mathrm{cm}^{2}$ ). All the patients used hormonal contraceptives before and during laparoscopic treatment. In patients of the third group dynamic laparoscopy was performed in 3 days after the first surgery. The duration of the dynamic laparoscopy was about $10 \mathrm{~min}$. In patients of the second group clinical uterine pregnancy rate was $42.5 \%$, the live birth rate was $82.4 \%$. In patients with distal tubal occlusive infertility and pelvic adhesions at stage III-IV uterine pregnancy rate was $16 \%$, the live birth rate was $50.0 \%$, extrauterine pregnancy were registered in $16 \%$ of women.

Conclusion: The described treatment strategy could be useful and effective method of the restoration the tubal patency in patients with tubal occlusive infertility and chronic salpingitis.

\section{FC_104}

The Essure ${ }^{\circledR}$ technique: confirmation visit at 3 months: the abdominal radiography and its interpretation. Animated 3D illustration.

J.B. Engrand, M. Boulogne, P. Delporte, P. Debodinance,

T. de Craecker, S. Nachot

${ }^{I}$ Centre hospitalier, Dunkerque, France, ${ }^{2}$ ART'FX Studi, Lille, France

The confirmation visit at 3 months is still one of the difficulties in using the $\operatorname{ESSURE}^{\circledR}$ technique. Currently in Europe, the only validated control in Europe is plain pelvic radiography. A hysterosalpingogram (HSG) should be used if there is the slightest doubt about tubal occlusion. In the USA, a HSG is always required.

The pelvic radiograph must always be compared with the surgical report, which must mention any difficulties encountered during placement: poor visibility, difficult tubal catheterization, or uterine malformation. It must also mention the number of coils visible in the uterine cavity, the position of the proximal end of the central axis, and the position of the PET fibers relative to the tubal ostia, as well as the duration of surgery. A duration exceeding $10 \mathrm{~min}$ suggests difficulties in placement. It is often an advance warning of failure, with a radiograph that is difficult to read.

Reading the radiograph requires attention. The difficulty is due to the $2 \mathrm{D}$ representation of a $3 \mathrm{D}$ image. The study of the position of the four markers of the must be meticulous: the distance between the inserts, the alignment of the markers, or their divergence as well as the position of the proximal marker of the external coils, relative to the other three markers. HSG is essential when the pelvic radiograph is unsatisfactory.

Different aspects of the most frequently encountered images are reproduced.

-Appearance of the inserts in a uterus that is anteverted, retroverted, in an intermediate position or deviated.

-Other less standard aspects that raise doubts about the positioning of the inserts are reported to be: inserts that are coiled, deviated, asymmetric, or in a reserve S shape. All of these cases are illustrated and the positions of the inserts are reproduced in animated 3D.

Most Essure ${ }^{\circledR}$ failures are due to failure to follow the placement protocol and poor interpretation of the confirmation at 3 months. Interpretation of the abdominal radiograph is not always easy. The radiograph visualizes the inserts, and the doctor must deduce their position from that. Ultrasound can usually localize the inserts and thus reduce the number of radiographs and HSGs. HSG is the only examination that proves tubal occlusion.

Can 3D ultrasound replace pelvic radiography in some conditions?

\section{FC_106}

Six years follow-up study pleading for Purizole's use or all hysteroscopics procedures

S. Seak-San, J.M. Foidart

Hôpital de la La Citadelle, Université de Liège, Liège, Belgium

Objective: Utilisation of Glycine (Glycocolle) for uterine distending media was associated with a high morbidity and a high mortality rate. Meeting these complications associated within the very known Turp Syndrome we have decided to replace Glycocolle by Purizole as a relative new distending media in gynecology. Since then, approximately all the above adverse sideeffects were completely removed from our "diary" complications. Design: We presented here our 06 years prospective follow-up study, from January 2002 to January 2008 and more than many thousand operated cases up to now (we will include for the final 
presentation, waiting the total data, the patients who were operated in December and January 2008). Indications, feasibility, complications, postoperative results will be presented and discussed below.

Results: Within these 06 years, many thousands patients were operated for symptomatic disagreement as metrorragia, menorragia, pelvic pain, and for some of them, the indications followed simply asymptomatic discoveries of endometrial lesions (patients under Nolvadex for example). The histological findings were notified as below: fibroids, endometrial atrophy, typical endometrial hyperplasia, atypical endometrial hyperplasia, endometrial atrophy, adenomyosis, no cancer was found in the samples collected. No complications described as a TURP syndrome were demonstrated among our population, as far as the etiopathology of this "disease" wasn't concerned with the metabolism of Mannitol-Sorbitol used. Resorption of the Mannitol-Sorbitol, thanks to the in-out bilan at the end of each hysteroscopic procedure was noticed. The different kind of analgesia required for all the procedures was also taken into account. Simple complications as persistent bleeding, pelvic aspecific pain, perforation, fever, more than one hospitalisation day for persistent epidural analgesia were encountered for the final data results. No major complications as conversion to laparotomy or secondary hysteroscopic procedures for hemostasis were noted.

Conclusions: Purisole is a safe and easy-to-use irrigations fluid for general hysteroscopy practice and it is particularly interesting, because avoiding the very high associated morbidity and mortality TURP syndrome. It's use is effectiveness and harmful as long as we respect some gently agreement with its use, as to follow the total quantity of fluid resorbed at each procedure, as long as we do know and recognize the potential hysteroscopic complications, and as long as we will perform that kind of surgery, whatever the distending media used, with surgeons in normal trainee able to detect abnormalities with hysteroscopic procedures.

\section{FC 107}

Long-term complications of office hysteroscopy T.C. van Kerkvoorde, S. Veersema, A. Timmermans St Antonius ziekenhuis, Nieuwegein, Netherlands

Introduction: Hysteroscopy is a widely used diagnostic and therapeutic procedure in gynecological practice. Many advantages have been described. During the procedure the complication rate is very low $(0.28-1 \%)$. However, little is known about the long-term complications, such as infection (Cochrane Review from 2006). The purpose of our study was to investigate the long-term complications of office hysteroscopy

Materials and methods: This study was set in a universityaffiliated teaching hospital with outpatient hysteroscopy facilities. Between January 2005 and October 2007, a total of 1,028 outpatient hysteroscopic procedures using a vaginoscopic approach were performed. The hysteroscopies were performed without any kind of anesthesia and without antibiotic prophylaxis. The complication registry of the gynaecology department was matched with the hysteroscopy registration. Additional a random sample of $330(32 \%)$ patients, that had undergone hysteroscopy, was taken of which medical charts were assessed for long-term complications during a period of 1 year after the hysteroscopy.

Results: Of the 1,028 hysteroscopic procedures, 622 (60\%) were diagnostic, and 328 (32\%) were therapeutic hysteroscopic procedures. $78(8 \%)$ of the procedures failed. In the complication registration no complications were registered. In the analysis of the medical histories we found one patient who returned 9 days after a therapeutic hysteroscopy to the hospital with mild signs of infection. Therefore the long-term complication rate was one of 1,028. (0.1\%) Discussion: Office hysteroscopy is a safe procedure. The risk of long-term complications is extremely low. 